\title{
Improving Solder Joints Formed in Microgravity by Use of Magnetic Soldering Paste Additives
}

\author{
Aaron Dunkle \\ adunkle@mix.wvu.edu
}

Follow this and additional works at: https://researchrepository.wvu.edu/etd

Part of the Other Aerospace Engineering Commons

\section{Recommended Citation}

Dunkle, Aaron, "Improving Solder Joints Formed in Microgravity by Use of Magnetic Soldering Paste Additives" (2019). Graduate Theses, Dissertations, and Problem Reports. 3863.

https://researchrepository.wvu.edu/etd/3863

This Thesis is protected by copyright and/or related rights. It has been brought to you by the The Research Repository @ WVU with permission from the rights-holder(s). You are free to use this Thesis in any way that is permitted by the copyright and related rights legislation that applies to your use. For other uses you must obtain permission from the rights-holder(s) directly, unless additional rights are indicated by a Creative Commons license in the record and/ or on the work itself. This Thesis has been accepted for inclusion in WVU Graduate Theses, Dissertations, and Problem Reports collection by an authorized administrator of The Research Repository @ WVU. For more information, please contact researchrepository@mail.wvu.edu. 


\title{
Improving Solder Joints Formed in Microgravity by Use of Magnetic Soldering Paste Additives
}

\author{
Aaron Dunkle
}

Thesis submitted to the

Benjamin M. Statler College of Engineering and Mineral Resources

at West Virginia University in partial fulfillment of the requirements

for the degree of

\author{
Master of Science \\ in \\ Aerospace Engineering \\ John Kuhlman, Ph.D., Chair \\ Terence Musho, Ph.D. \\ Edward Sabolsky, Ph.D.
}

Department of Mechanical and Aerospace Engineering

Morgantown, West Virginia

2019

Keywords: microgravity, composite solder, electronics repair

Copyright 2019: Aaron Dunkle 


\title{
Abstract \\ Improving Solder Joints Formed in Microgravity by Use of Magnetic Soldering Paste Additives
}

\author{
Aaron Dunkle
}

With the expansion of the space industry, the need for the capability to repair electronics in space grows. Previous researchers have found that attempts to solder in microgravity have yielded joints of poorer quality than those made on Earth, with solder joints formed in microgravity having increased interior porosity. WVU's microgravity research team (MRT) constructed an experiment to solder onboard a microgravity aircraft flight with the purpose of exploring this problem and to test out a possible solution. MRT's testing collected solder samples both in microgravity and a ground control test, which were then cross-sectioned to allow porosity of the interior of the joints to be studied. This determined that there was an increase in porosity for the joints formed in microgravity. Exploration into the source of the gases in the solder that contributed to porosity was attempted by energy dispersive $\mathrm{x}$-ray spectroscopy analysis of the interior of the voids left behind by bubbles in the joints. Although carbon, an indicator of flux vapor, was indeed found in the voids, this examination proved inconclusive due to the possibility of contamination during the process of cross-sectioning the joints. In an attempt to reduce the increase in porosity seen in microgravity solder joints, some of the MRT solder samples utilized solders that had iron microparticles added and a magnetic 
field below the circuit boards that were being soldered to provide a magnetic body force on the solder. This replaced the gravitational force and encourage the flow of bubbles in the solder toward the top of the joints where they could escape to the atmosphere. The magnetic manipulation of the solder did not provide any consistent, statistically significant change to the average porosity of the joints. Magnetic manipulation of solder could be further explored with changes to variables such as magnetic field strength and melt time of the solder, however the current work has not shown a benefit to the quality of the joints. 


\section{Acknowledgements}

I would like to thank those who have been involved throughout the course of this research as well as those who made it possible.

My thesis advisor Dr. John Kuhlman for giving me the opportunity to work on this project and for the help and advice he provided.

WVU's Microgravity Research Team for developing the ideas that led to this research and for their work in developing the experiment. Especially those who first conceived this idea: Gina Eberhart, Steven Itschner, and Jacob Cordonier.

NASA's USIP program and WV Space Grant Consortium for funding this research and providing technical and administrative support.

Mrs. Kathy Sabolsky who assisted MRT and me in all materials science matters. 


\section{Table of Contents}

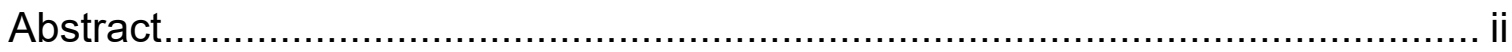

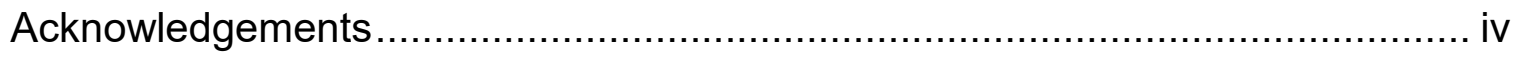

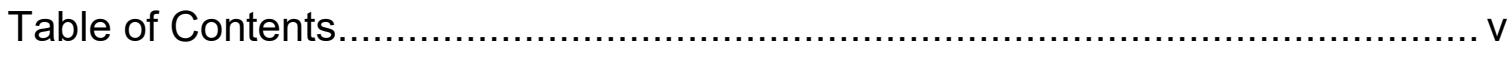

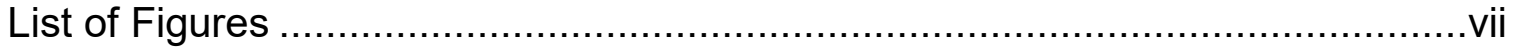

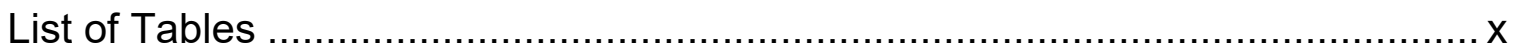

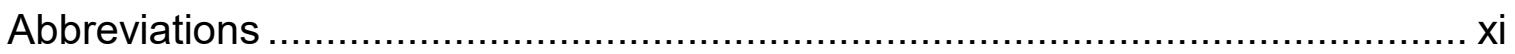

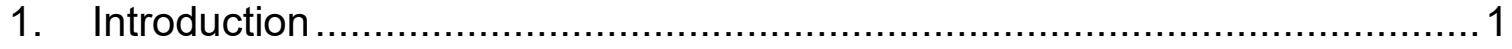

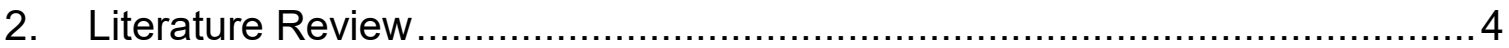

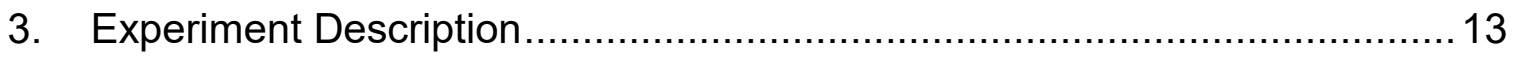

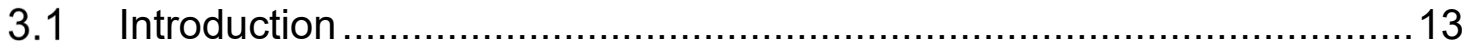

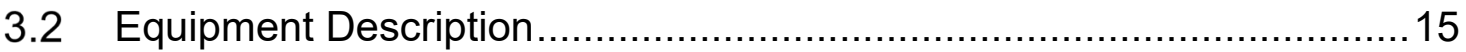

3.3 Flight Conditions and Procedures ….................................................. 31

3.4 Data Acquisition and Reduction .................................................... 41

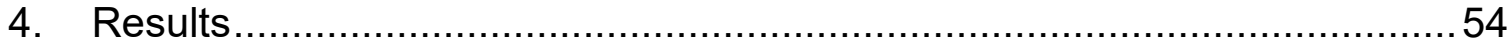

4.1 Vision Inspection (First Round Qualitative Analysis)..........................54

4.2 Samples from reflow ovens and induction heating..............................62

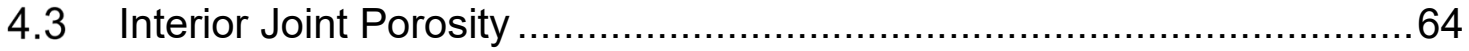

4.4 Energy Dispersive X-ray Spectroscopy ….................................... 70

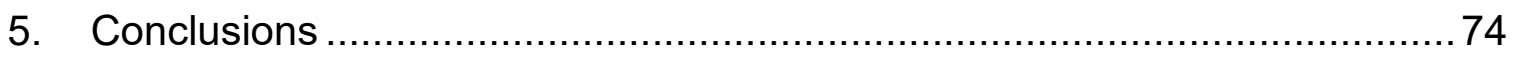

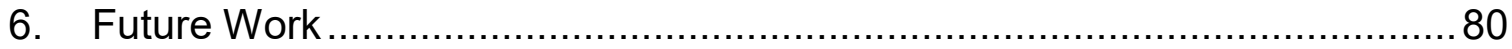

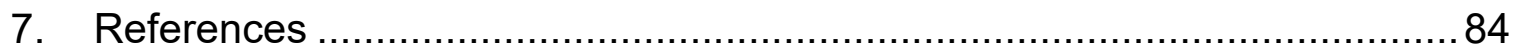

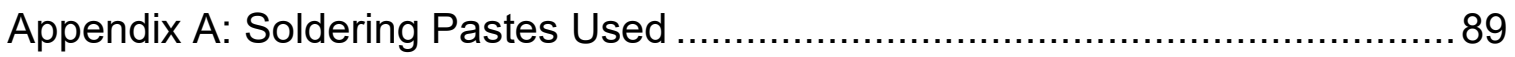

Appendix B: Parabolic Aircraft Gravity Data ……......................................... 90

Appendix C: Hand Soldering Visual Inspection.............................................. 93

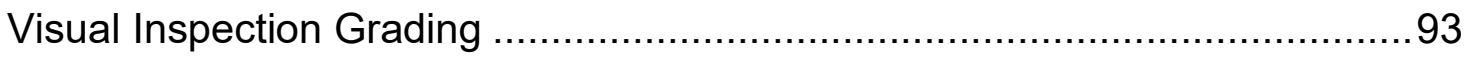

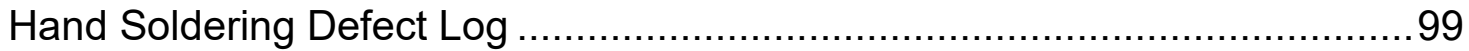

Appendix D: Visual Comparison of Microscope Images vs Processed Images with

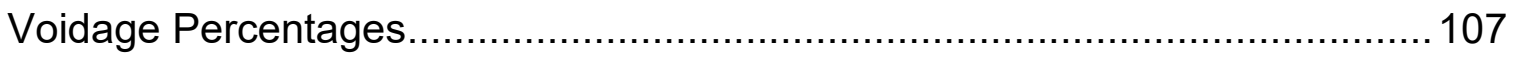

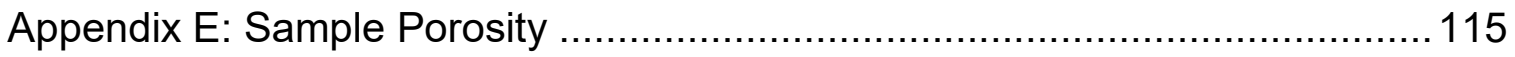

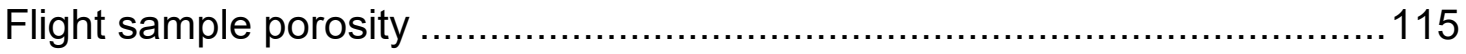




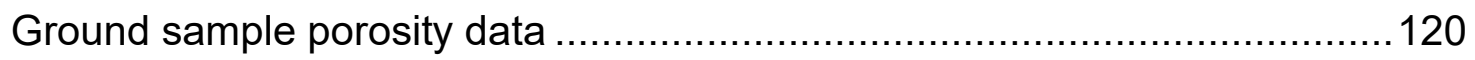

Appendix F: Magnetic Field Plots and Data .............................................. 123

Appendix G: Matlab Image Processing Code ........................................... 126

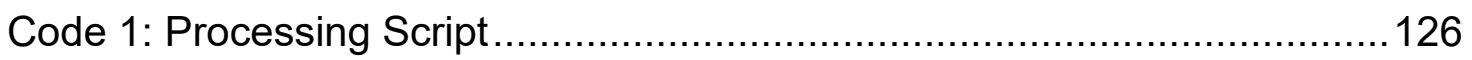

Code 2: Image Preprocessing Function ............................................... 127 


\section{List of Figures}

Figure 1: Magnetic particle alignment under a magnetic field [19] ....................

Figure 2: Creep rates of composite solders [13] ........................................ 10

Figure 3: Illustration of placement of solder and resistors on sample boards .....18

Figure 4: The hand soldering section of the payload with the 3 originally planned

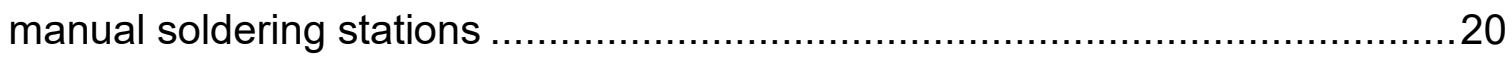

Figure 5: Hand soldering sample box showing placement of boards and magnets

Figure 6: Magnetic field intensity on a hand soldering board, in this case varying from 620 Gauss (dark red) at the center of the board to 150 Gauss in the farthest

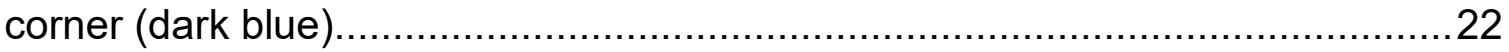

Figure 7: Magnetic field decay of a single hand soldering box magnet as a function

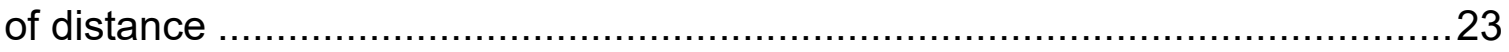

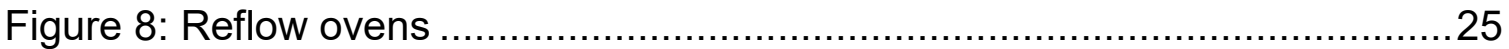

Figure 9: Placement of oven magnet above sample board when folding arm is

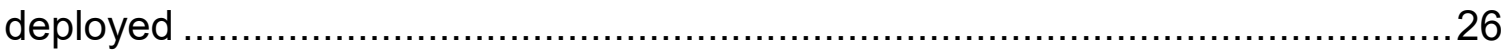

Figure 10: The induction heating system as configured for ground testing .........28

Figure 11: The payload assembly in flight configuration ..................................31

Figure 12: The vertical acceleration experienced by the payload .....................35

Figure 13: Gravity levels for one set of flight parabolas, parabolas 16 through 20

Figure 14: Average gravity level measured during each microgravity period......36

Figure 15: RMS of gravity level measurements during each microgravity period 37 
Figure 16: Length of microgravity periods in flight........................................

Figure 17: A hand-soldering sample box after flight, prior to board cleaning ......42

Figure 18: A hand soldering board before cleaning .....................................43

Figure 19: Illustration of cutting lines on a hand soldering sample board...........44

Figure 20: An example of the grading of joints in a sample box......................46

Figure 21: An example log of defects showing what errors were present in joints marked defective

Figure 22: Microscope images of a solder joint. An optical microscope image (left) and an SEM image (right) both at $40 x$

Figure 23: A microscope image and its tricolor counterpart ............................52

Figure 24: Effects of soldering iron temperature on quality of joints .................55

Figure 25: Quality of joints created by solderers on ground and in flight as percentage of total possible joints in each grade 56

Figure 26: Quality of joints comparing flight and ground for solderers as percentage of joints they produced. .57

Figure 27: A joint from the induction heating system featuring a large void just beneath the top surface .64

Figure 28: An example of the output from EDS, an image of the area being observed and the mapping of atomic species detection .71

Figure 29: The area mapped in Figure 28, but showing only the presence of carbon 72

Figure 30: EDS carbon map from the interior of a large void, 20\% Carbon .73 
Figure 31: EDS carbon map from the polished surface of the same joint that Figure

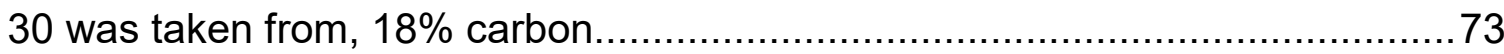

Figure 32: A model of an Arcan disk that could be used be used to test the strength

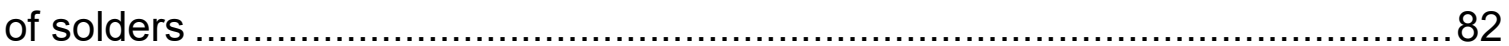




\section{List of Tables}

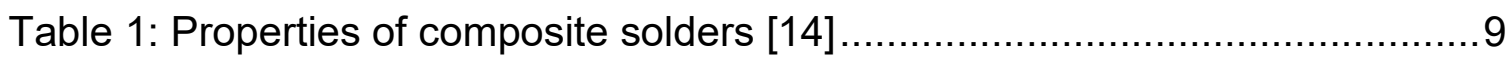

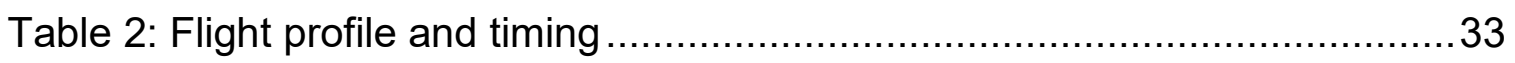

Table 3: Length of microgravity periods in flight............................................38

Table 4: Hand soldering defects observed in samples and their description ......48

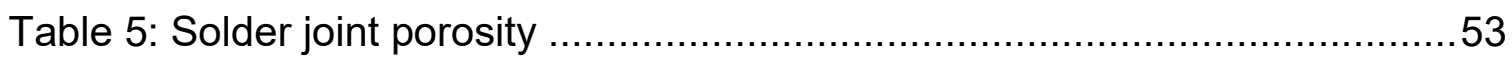

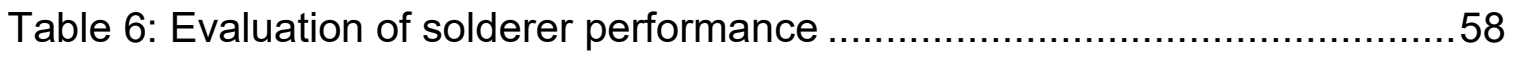

Table 7: Number of solder samples in each category ....................................60

Table 8: Number of solder samples in each category, sorted by visual quality...61

Table 9: Percentage of joints from each category that fall into each quality .......62

Table 10: Effect of soldering iron temperature on the voidage of joints within each

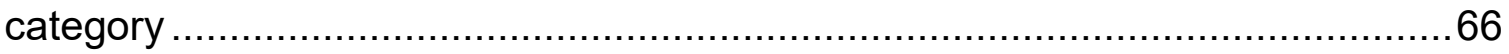

Table 11: Average percent voidage based on temperature and solder only .......66

Table 12: Average joint porosities in each sample box.................................67

Table 13: Average joint porosity for each category of solder and environmental

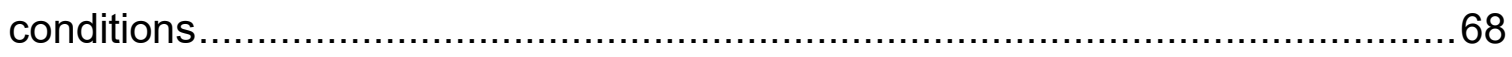

Table 14: Standard deviations of averages presented in Table 13 ..................68

Table 15: Interior voidage percent compared to visual joint quality ...................69 


\section{Abbreviations}

CLEAR

DAQ

EDS

ISS

LMP

MRT

NASA

ORU

PW

SEM

SMiRF

SoGRE

RMS

UTS

WVU

Zero-G
Component-Level Electronics-Assembly Repair

Data Acquisition

Energy Dispersive X-ray Spectroscopy

International Space Station

Low Melting Point (solder)

Microgravity Research Team

National Aeronautics and Space Administration

Orbital Replacement Unit

Powerful Wetting (solder)

Scanning Electron Microscope

Small Microgravity Research Facility

Soldering in a Reduced Gravity Environment

Root Mean Square

Ultimate tensile strength

West Virginia University

Zero Gravity Corporation 


\section{Introduction}

As space missions and satellites continue to grow in number, the need for solutions to the problems faced grows. One such problem is the issue of electronics repair on satellites and spacecraft. Long duration space missions face deterioration of electrical components just as electronics on Earth do. Replacement of electronics on satellites is a costly and time-consuming process, as in many cases any component that malfunctions needs to have a replacement flown up from the ground. Having the ability to solder in microgravity environments would provide the capability to repair electronics components and circuitry would save the time and money otherwise invested in these repairs. In addition, this capability could reduce the need for space circuit boards on future manned space missions, which would help reduce launch mass and cost.

In prior research into soldering in microgravity, it is commonly found that solder joints formed in the absence of gravity are of inferior quality to those formed under normal gravity conditions. Studies conducted in various microgravity environments: space station, parabolic aircraft, and drop towers, have found that solder joints formed in microgravity have a higher interior porosity compared to joints created in a normal gravitational environment. This causes microgravity solder joints to be mechanically weaker and less electrically conductive.

As one possible solution to this problem members of West Virginia University's Microgravity Research Team (MRT) proposed using solders that have 
iron particles as additives and soldering within a magnetic field. Ideally, this would create a nearly uniform force within the molten solder that would act as a body force on the solder, replacing the buoyancy force caused by Earth's gravity and allowing for the solder to flow around the vapor bubbles, forcing these bubbles to the surface of the joint where they would be released from the molten solder.

For this experiment, microgravity conditions were achieved via a parabolic aircraft flight contracted through the Zero Gravity Corporation (Zero-G) onboard GForce One, Zero-G's modified Boeing 727, through flying parabolic arcs that provide a period of approximately 20 seconds of microgravity per parabola. Three methods of soldering in this restricted time frame were designed; traditional hand soldering stations, a pair of reflow ovens, and an induction heating system. These methods were used to generate control samples through a simulated flight in the lab using a video from a previous flight to mark the timing of beginnings and ends of microgravity periods. Then the hand soldering stations and reflow ovens were placed onboard G-Force One to acquire samples under microgravity conditions for a series of 30 parabolic arcs.

During the flight and ground testing, there were solder joints made both in a magnetic field and outside the presence of a magnetic field. Additionally, six different solder compositions were used; three iron percentages added to two different solder pastes used as a base. The samples were assessed based on exterior surface quality of the joint, as well as by the interior porosity, so that it 
could be determined if the solder pastes with iron dispersoids, used in the presence of a magnetic field were successful in producing better quality joints when compared to joints made using solder pastes without iron additives, or those soldered outside magnetic fields. 


\section{Literature Review}

Soldering is the process of attaching two metals together by use of another metal, solder, as a filler at the joint between the items being connected. By definition, solders have lower melting points than the metals they connect and work by bonding to the surfaces of the joint and filling in any space to create a solid connection. This type of attachment is most commonly found in electrical circuitry, as the metals that make up solders can both attach components together or to a circuit board with strength sufficient in most applications, and also conduct well enough to not impede the flow of electricity. Traditionally solders used for electronics were composed of a lead alloy, usually lead-tin, but due to health and safety concerns, lead solder use is becoming less common. Lead-free solders now being used tend to instead favor tin and silver.

Soldering in microgravity environments has been the focus of study by several research groups previously, both to study the differences between solder joints made in microgravity compared to those made under normal gravity conditions, and to explore the potential benefits of soldering during space missions. Although the international space station (ISS) has a soldering kit onboard, in-situ repairs are not the standard practice when there is an electronics malfunction onboard $[1,2,3]$. Instead, the station carries modular units that group a system of electronics together, called orbital replacement units (ORU). When an electronics problem is encountered, the ORU is removed and replaced by a similar unit stored onboard. Then the entire unit is sent back to Earth where it is assessed, 
repaired, then repackaged and sent back to the ISS as a backup for the next time an error is encountered $[3,4,1]$. This presents problems, in that a large part of the costs of space missions is the launching of spacecraft and materials into orbit, and as the weight of the launch increases the cost does as well. Additionally, as space missions go farther beyond Earth it becomes more important that they are independent. Future deep-space missions will not have the option of relying on being able to send components to Earth for repair as it will become imperative that space missions be able to respond to issues as the arise on their own and in a timely fashion. Pettegrew et al. stated that, "The flexibility to respond decisively to unforeseen problems is a crucial issue for deep-space missions." [2]

NASA has explored other options to eliminate the need to transport ORUs to Earth when repairs are needed. One option is the possibility of taking a single electronics card from an ORU to be repaired; another is giving astronauts the ability to do repairs in orbit. This was the focus of NASA's CLEAR project $[5,4]$, developing a system where electronics could be evaluated, diagnosed, and repaired onboard the spacecraft they were used on.

The concept for CLEAR was based on the Gold Disk system that US Navy ships carry for emergency on-ship repairs, where a single component in an electrical system can be identified as the cause of a malfunction and replaced or repaired [4]. Diagnosis of problems was done by reading electrical signals between two nodes in a circuit and comparing that signal to the signal reading of a similar 
circuit in working order. Accola [6] estimated in 1990 that increasing the percent of repairs astronauts do to $30 \%$ of total electronics repairs would reduce resupply upmass by as much as $20 \%$ while only reducing their available time by $2 \%$.

Although repairing electronics in space would reduce the overall weight and cost of missions, there are problems that must be understood before it can become the primary method of repair. Solder joints formed in microgravity are generally found to be of lesser quality than those made on Earth. One of the primary reasons behind this is that microgravity solder joints tend to be more porous and therefore weaker joints and more likely to break under stress and thermal cycling.

Imperial College London flew an experiment onboard a parabolic aircraft flight designed to test the strength of microgravity solder joints made under microgravity conditions. This experiment found that interior porosity of joints increased from $1 \%$ to $14 \%$ and that the strength of the connection decreased by $32 \%$ for joints formed during the flight as compared to those formed under normal gravity conditions [7].

A series of parabolic aircraft flights conducted by NASA between 2001-2004 [8] investigated the porosity of microgravity solder joints using several solder and flux combinations found that when comparing porosity from microgravity joints to those formed in $1 G$, all combinations had higher porosity at lower gravity levels. This study also noted that the location of voids changes in microgravity, because 
in normal gravity buoyancy forces gas bubbles toward the upper surface of the solder where they will flow out. However, in microgravity, there is no buoyant force to direct the bubbles, so they tend to stay in place rather than flowing out and form voids when the solder solidifies. Although, on aircraft there is some unsteadiness in the gravity level, called G-jitter, due to vibrations on the plane and atmospheric conditions. Because of this fluctuation in gravity level, there are small accelerations of the bubbles that switch direction and therefore switch the direction of movement of bubbles. This results in higher porosity near the center of the joint and lower porosity near the joint's surfaces, where these small movements are sufficient to draw bubbles out of the molten solder.

Another study that NASA, called SoRGE [9], studied soldering onboard ISS, which would be a truer microgravity than a parabolic aircraft flight. SoRGE evaluated solder joints first by a visual inspection of the joints, then by CT scans of the interior to determine the voidage. Like the other experiments, SoRGE also found that the joints formed with lower gravity levels had higher porosity on average.

The sources of the voids in solder are not yet fully understood. They are thought to be caused, primarily, by gasses from vaporized flux becoming trapped in the solder as it solidifies $[8,9,10]$. Another suggested source of the gas bubbles that become voids in solder, is that they come from the circuit boards themselves. The voids may result from water absorbed by boards releasing as they heat [9], to 
introduce moisture into the joint that will form gas bubbles in molten solder. This idea is supported by results from Watson, et al., which showed reduced porosity in solder joints made on circuit boards that had been baked prior to being soldered. Specifically, a reduction from average internal porosities of $11.0 \%$ and $3.21 \%$ in $0 \mathrm{G}$ and $1 \mathrm{G}$, respectively, to $6.32 \%$ and $2.78 \%$ after the boards were baked for a period of 4 hours to demoisturize [8].

There are ways to mitigate the weakening of the joint due to increased porosity in microgravity. The use of small particles mixed into solder pastes has been found to increase the strength of solder $[11,12,13,14,15]$; solders that include these dispersoids are referred to as composite solders. Composite solders have been found to improve many properties of solder. Generally adding dispersoids into a solder is found to improve the mechanical strength of the joints made using that solder $[16,11,17,18,19,14]$. Calabro et al. found that using iron particles as the dispersoids they were able to increase the ultimate tensile strength by nearly $50 \%$, as is shown in Table 1 [14]. Table 1 shows some of the negative effects that large amounts of dispersoids can cause in solder. Although the strength increases with the addition of more iron, the conductivity of the solder decreases, and the contact angle increases, which is indicative a solder with poor wetting. In addition to including dispersoids in solders, they can be further improved by aligning the particles within the solder by solidifying the mixture in the presence of a magnetic field [14, 19]. Aligning magnetic particles within the solder tends to chain the particles together as their magnetic poles will face the same 
direction exposing the opposite pole to the particle adjacent to it [19]. These particle chains can prevent the dispersoid from settling out of the molten solder or rising to the surface of the solder. This property is illustrated schematically in Figure 1, which shows the alignments that would be expected for relatively large or small dispersoids [19].

Table 1: Properties of composite solders [14]

\begin{tabular}{|c|c|c|c|c|c|c|}
\hline Sample (SnAg + wt.\% Fe) & Modulus (MPa) & UTS (MPa) & Elongation (\%) & Conductivity $\left(\mathrm{M} \Omega^{-1} / \mathrm{m}\right)$ & Hardness (HV) & Contact angle ( $\left.{ }^{\circ}\right)$ \\
\hline $0 \% \mathrm{Fe}(\mathrm{SnAg})$ & 5158 & $34.5 \pm 2.2$ & $25.7 \pm 7.4$ & $9.44 \pm 02$ & $15.74 \pm 47$ & $12.1 \pm 1.7$ \\
\hline $5 \%$ & 4992 & $36.8 \pm 1.6$ & $15.0 \pm 1.6$ & - & $16.86 \pm 54$ & $11.3 \pm 0.9$ \\
\hline $5 \%$ aligned & - & $48.7 \pm 1.2$ & $7.7 \pm 2.1$ & - & - & - \\
\hline $10 \%$ & 7977 & $44.8 \pm 3.4$ & $5.5 \pm 1.0$ & $8.32 \pm 10$ & $16.34 \pm 66$ & $11.7 \pm 1.7$ \\
\hline $10 \%$ aligned & - & - & - & $7.23 \pm 16$ & - & - \\
\hline $20 \%$ & 9564 & $50.9 \pm 0.6$ & $4.7 \pm 1.4$ & $6.43 \pm 18$ & $27.15 \pm 76$ & $23.4 \pm 1.5$ \\
\hline SnPb (literature) & - & - & - & $5.58(7)$ & $13.1(7)$ & $16(7)$ \\
\hline
\end{tabular}

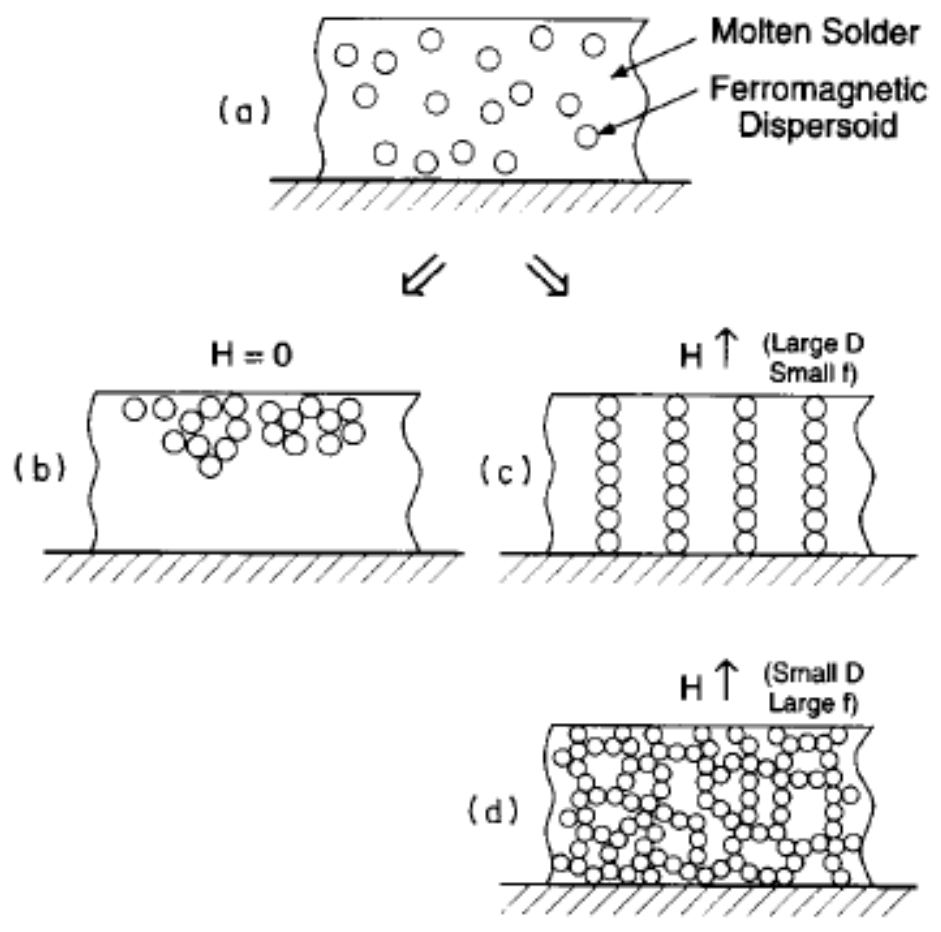

Figure 1: Magnetic particle alignment under a magnetic field [19] 
A primary reason for using composite solders is to produce joints that are more resistant to creep or deformation under continual stress [13, 12, 19, 17]. Figure 2 shows data from a creep test of solder samples. The composite solders have significant strain resistance when compared to their base solder. This creep resistance is often sought after for sensitive applications such as lasers and fiber optics [13], but is a desirable quality in all solder joints. The reduction in creep is due to the dispersoid acting as an obstacle to grain boundary sliding and deformation [12]. By including dispersoids in the solder, these particles act as reinforcement to support the solder adjacent to the particle and spread stresses more evenly throughout the solder.

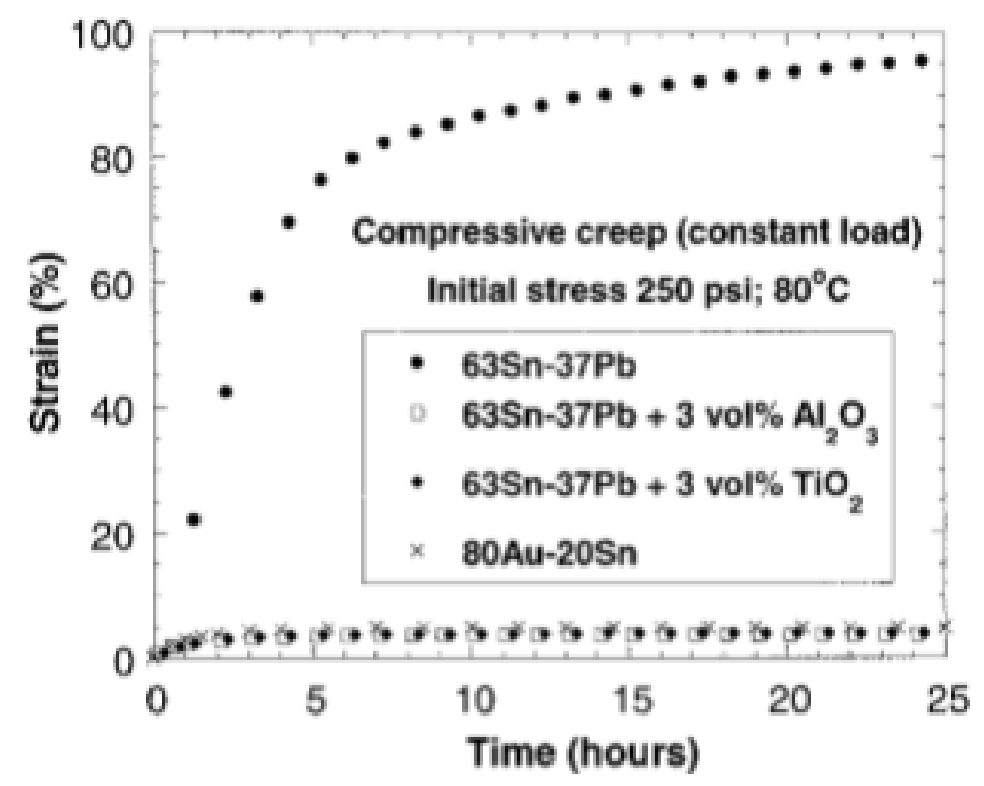

Figure 2: Creep rates of composite solders [13]

There are some known drawbacks to the use of composite solders. For example, as seen in Table 1 as the amount of dispersoid increases, the electrical 
conductivity can be reduced and the wettability of the solder decreased [14]. In addition to these possible deficiencies, the addition of dispersoids into solder can increase the voidage in solder joints $[17,19,20]$. The particles interfere with the flow path of vapor bubbles in the solder and make it more likely that they are trapped in the joint during solidification.

Using dispersoids has, in some cases, been found to increase solder joint voidage, which is already increased in microgravity. However, if the dispersoids are magnetic, they can be potentially used to manipulate solder into flowing into joints more easily. A study from Yale used iron additives in a solder to manipulate the flow direction of solders [14]. By melting solder in the presence of a magnetic field they were able to direct solder into channels and even flow solder upward into channels above the molten solder under Earth gravity. This indicates that the magnetic pull on the dispersoid can act as a body force to the solder as a whole. Since the cause of voids in microgravity is a lack of buoyant force to push the bubble out of the solder, magnetic force may be able to replace gravity as a body force that would allow buoyant movement of the bubble and help mitigate void formation in microgravity solder joints. This is the key hypothesis of the current research.

The concept of using a magnetic solder soldered in a magnetic field in microgravity was first conceived by the WVU's Microgravity Research Team students in 2014 [21]. In keeping with tradition of the MRT, this research area was 
chosen by the students for an experiment that could be developed and flown on a microgravity aircraft. However, due to cancelation of the NASA student flight program the group that had originally developed the concept was no longer attending the university by the time that the experiment was flown in November 2017. As an alternative, WVU MRT constructed a microgravity drop tower on-site at West Virginia University, so that microgravity experiments could be conducted.

The drop tower, the Small Microgravity Research Facility (SMiRF), allows for 1.25 seconds of reduced gravity during free fall from the tower during which experiments designed for microgravity can collect data [22]. Early tests of MRT's microgravity soldering experiments were performed using SMiRF; it was during this time that the first magnetic solders used by MRT were created [23]. Following this work, the first aircraft flight experiment for the microgravity soldering systems was attempted by MRT, using a small aircraft, a Cessna 172, performing parabolic arcs to reach reduce gravity conditions. The aircraft flight allowed for the soldering to be performed by a human operator, rather than an automated system, and gave a longer period of reduced gravity than SMiRF, up to 2 to 3 seconds, during which the soldering took place [23]. However, both this aircraft flight and the SMiRF experiments were conducted without the use of magnetic fields during soldering. The research present in this document is a continuation of this research and has been performed in conjunction with ongoing experiments performed by the current MRT students. 


\section{Experiment Description}

\subsection{Introduction}

The experiment conducted consisted of two major data gathering events after preliminary development and testing of the experimental payload. Event 1 was a ground test of the complete payload prior to the microgravity flight to gather control data. Event 2 was the microgravity flight which collected data to test the hypotheses about magnetic soldering and microgravity solder porosity.

The experiment was designed to be flown on G-Force One, Zero Gravity Corporation's microgravity aircraft. Five flyers were onboard the aircraft as per Zero-G standard; these flyers were selected from the Microgravity Research Team students to operate the experiment and create solder samples. The flight took place in Sanford, FL, and consisted of a series of 30 parabolic arcs conducted in sets of 5 , each giving a microgravity period of about 15 to 20 seconds. The first set of these parabolas were not true zero-G, but Martian or Lunar gravity level parabolas to ease the flyers into the reduced gravity environment. After the initial set of low gravity parabolas, the remaining five sets were zero-G.

The flight payload consisted of 5 soldering stations, one for each flyer, comprised of 3 soldering methods: hand soldering irons (3), a pair of reflow ovens, and an induction heating system, as well as a flight data acquisition and an air filtration system. This payload was contained in an aluminum payload frame fitted with polycarbonate panels on the sides and top and an aluminum baseplate for 
mounting to the aircraft floor. The polycarbonate side panels had access holes cut into them with plastic gloves mounted to these holes to allow flyers to operate the experiment while still containing any fumes that were produced by soldering inside the payload.

Before the flight, a full test of the experiment in flight condition was performed. Using a video of a previous flight for timing of parabolas, a full 30 parabola test was conducted. The solder samples (solder joints) created during this test were used as control data and are referred to in the present analysis as ground samples. In addition to gathering data, this test also served to train the flyers in maneuvering and manipulating their equipment with the restrictions of using gloves and access holes inside the payload. The videos recorded during this flight also served to prove that the filtration system operated adequately.

Between the ground test and the flight, the induction heating system was found to be unsafe for flight due to a leak in its cooling system and was removed from the payload. It was replaced by an additional soldering iron station and its operator retrained to operate this iron. Other systems remained unchanged between ground and flight testing, so the final flight configuration of the payload contained a pair of reflow ovens and four hand soldering stations. Four of the five flyers were the same as during the ground test, the oven operator, two of the hand solderers, and the induction system operator who was converted to a hand 
solderer. The latter, however, was replaced during the flight with a substitute after the first set of parabolas due to air-sickness.

Upon completion of the flight, the sample boards and experiment were transported back to WVU for processing and analysis. This included cleaning sample boards, inspection of solder joints, cross-sectioning and polishing of the joints, and microscopy observations. The overall goal of the data analysis was to determine solder joint quality based both on external visual appearance and interior porosity, to determine the effect of microgravity conditions on solder joints and to test the hypothesis that soldering in a magnetic field can replace gravity as the missing body force on these joints and allow for buoyant flow of the molten solder, thereby reducing the joint porosity.

\subsection{Equipment Description}

\subsubsection{Solder pastes}

For the experiment conducted during the present research, six different solder pastes were used; these consisted of two basic pastes each with three concentrations of iron microparticles: $0 \%, 4 \%$ and $6 \%$ by weight. Both base pastes were commercially available lead-free solders produced by the Koki Company, one solder paste (T4AB58-M742) is comprised mainly of tin and bismuth and has a relatively low melting point and was given the designation LMP. The other (S3X58M501) is comprised of primarily tin, with smaller percentages of silver and copper, 
and was designed to have enhanced wetting properties, this was designated powerful wetting, PW.

Pastes with iron additive were made in $50 \mathrm{~g}$ batches, by adding a premeasured amount of 325 mesh iron powder, approximately 44 micron diameter particles, to either 47 or 48 grams of solder paste, for $6 \%$ or $4 \%$ iron solders respectively. The pastes were then stirred with a sonic stirring wand for a period of approximately 10 minutes, with intermittent pauses during that time to prevent the solder from heating to a point that the flux would evaporate. The final products were labeled by adding their iron percentage to base paste's abbreviation, for example, 'LMP4', and this naming convention was continued to the mapping of which pastes were used in sample boxes and data analysis, and thus can be seen throughout the results section of this document.

The base pastes that were chosen for the experiment were selected based on testing by MRT 13, who tested by soldering with several pastes and decided which to use based on qualitative parameters of the solder, including wicking into through holes on the sample boards, wetting to resistor wires, and ease of manipulation with soldering irons.

Other iron percentages were also tested before deciding on $4 \%$ and $6 \%$. But at higher iron concentrations, the particles tended to clump together and rise out of the paste, and at lower concentrations the pastes were not as susceptible 
to the presence of magnetic fields. The $4 \%$ and $6 \%$ iron pastes were sufficiently magnetic that the pastes were observed to react to magnetic fields, but low enough that the iron remained in the paste during storage and melting of the solder. At these iron percentages, the unmelted solder can be moved by magnets as it is being injected from the syringe, and melted joints can support the weight of a sample board.

\subsubsection{Sample Board Setup}

Before each test, all the sample boards that were to be used in that test were prepared. This included determining which solder pastes would be used during the test, mapping their locations on the boards, and applying the pastes. Each melting system used a similar board setup for each test. The hand soldering used five pairs of resistors per Sparkfun board, each pair being used for one parabola and five pairs so that in flight each board would be used for a set of parabolas, switching to a new board during breaks in parabolas. The reflow oven and induction sample boards used one half of the boards for samples. The back half was clamped onto secure each board during heating, since for these systems the boards were replaced with a new board between parabolas. An example of how the boards were set up is shown in Figure 3. 


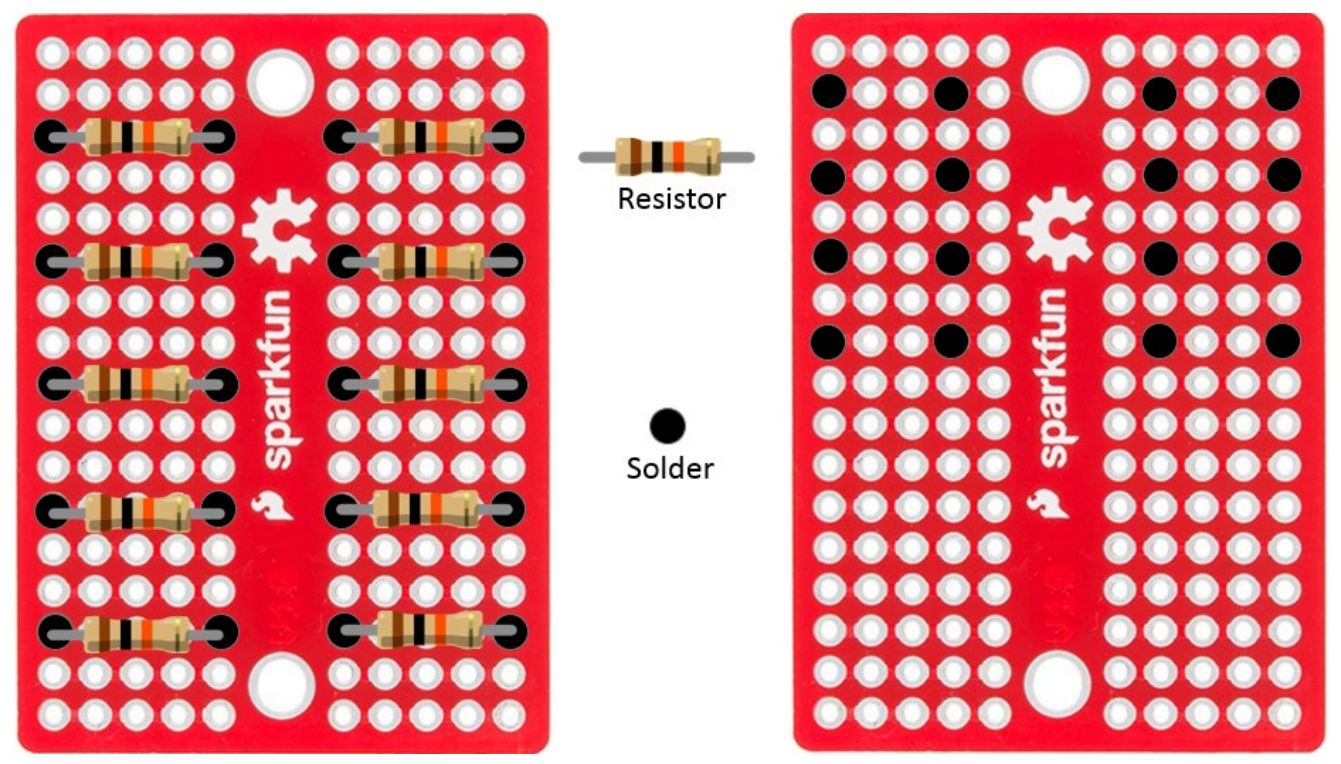

Figure 3: Illustration of placement of solder and resistors on sample boards

The paste was applied to the designated locations by filling syringes with the chosen paste and ejecting it to the top of the board around the wires of resistors that were already inserted into through-holes in the boards (left side of Figure 3), except in the case of the reflow ovens, which did not include resistors on the boards (right side of Figure 3).

The pastes used require refrigerated storage, so boards were prepared as close to the time of use as possible. In all ground testing, this meant board preparation immediately before tests; however, due to limited preparation time available the day of the flight, flight boards were prepared the night before and left in a refrigerator overnight. They were then transported to the airport in a cooler and moved to the payload during the last loading period before boarding and takeoff. 
Each soldering station had a preplanned layout of solder pastes; a chart showing which joints were made from each paste can be found in Appendix A: Soldering Pastes Used. The general trend for this was that hand soldering iron used LMP solder for the first half of the flight (and simulated flight for ground testing) then PW solder for the second half, while the reflow oven and induction system primarily used one of these two base pastes, LMP and PW, respectively. The iron percentages were changed every parabola to distribute them throughout the sample groups.

\subsubsection{Hand Soldering Stations}

Hand soldering was the primary method of sample creation during the experiment, as it is the most reliable and most controllable method of melting solder, and because an in-space electronics repair system would likely take the form of hand soldering kits operated either by human astronauts or robotic solderers. For this reason, it was initially planned that three of the five flyers would operate hand soldering stations. This later became four of five flyers when the induction system's cooling system leaked. Extra hand soldering stations were manufactured by the research team and brought with the payload to the flight location as emergency backups for any soldering system that had an unforeseen failure. An image of the hand soldering section of the payload, showing the manual soldering station setups can be seen in Figure 4. 


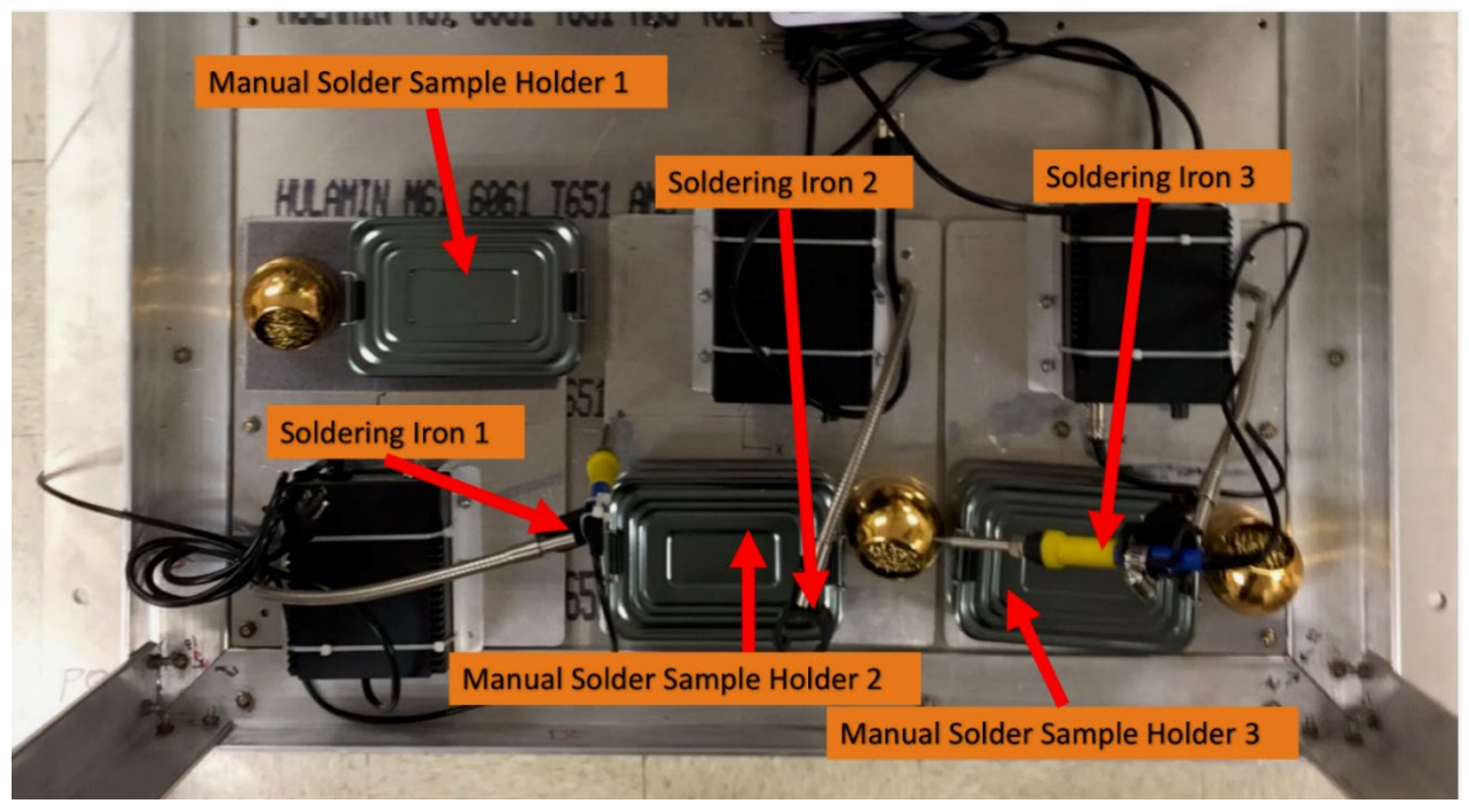

Figure 4: The hand soldering section of the payload with the 3 originally planned manual soldering stations

Each of the soldering stations onboard the payload includes: a soldering iron with an adjustable power supply for temperature regulation, a gooseneck microscope holder converted to hold soldering irons to limit their movement during microgravity periods, a coiled brass soldering tip cleaner, and a box of solder samples, all secured to a 12.25 " $\times 10 " \times 1 / 8$ " aluminum mounting plate that was then bolted to the payload's base plate.

To test the effects of magnetic fields on the iron solders, some sample boxes contained magnets while others did not. One of three boxes used in ground testing had magnets, and three of four boxes in flight used magnets. Each box contained six sample boards with five rows of two resistors, which allows for up to 120 samples to be created per box. These boards were mounted in the boxes via 
non-metalic standoffs. Using standoffs allowed space for magnets to be placed under the boards. Figure 5 shows a partially assembled sample box where the placements of boards and magnets can be seen. In boxes that contained magnets, foam insulation was used to keep the spacing of the magnets while they were placed in the boxes, so they could not contact each other when being set in place. Once in place they were epoxied to the bottom of the box to permanently secure their positions.

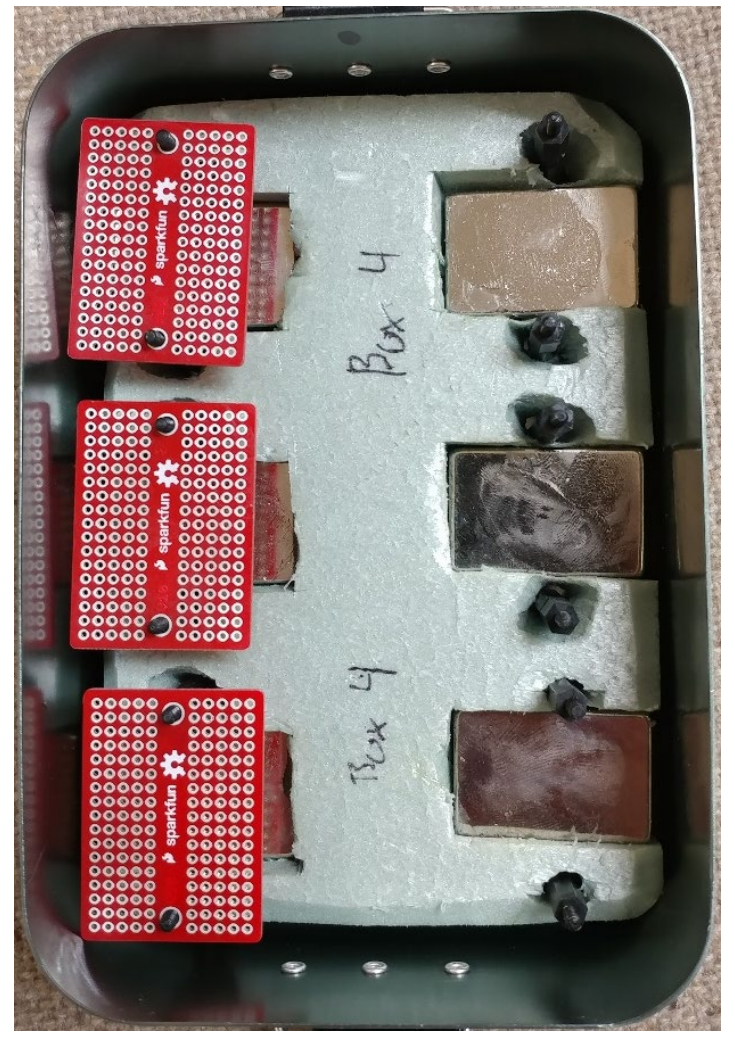

Figure 5: Hand soldering sample box showing placement of boards and magnets

The magnets used in the hand soldering sample boxes are 1 "x 1.5 " $\times 0.5 "$ neodymium magnets, each have a nominal magnetic strength of approximately 
2000 Gauss at its top surface (as shown in Figure 5). This strength decays logarithmically away from the magnet face. Because of this, soldering locations on the board not directly above the center of the magnet experienced less magnetic force. A plot of the measured magnetic field strength that a board was exposed to during soldering is displayed in Figure 6 and Figure 7 shows how the magnetic field of a single magnet decays with distance from the face of the magnet.
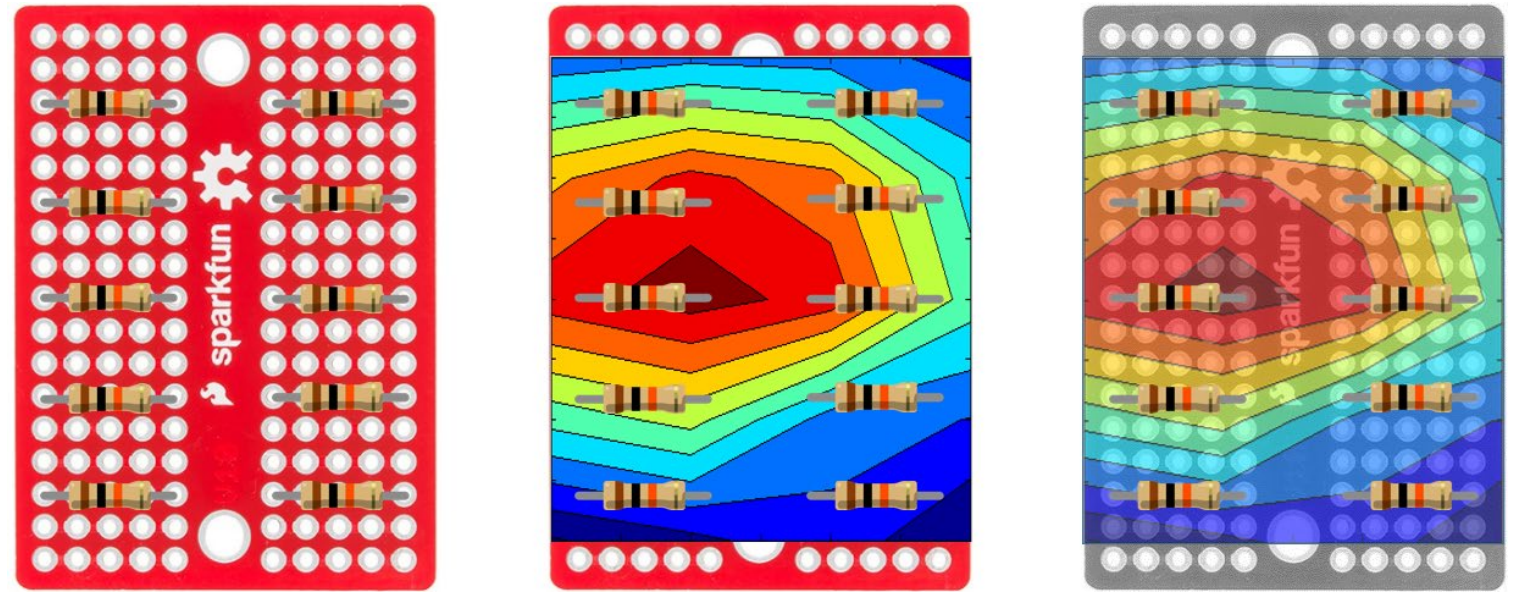

Figure 6: Magnetic field intensity on a hand soldering board, in this case varying from 620 Gauss (dark red) at the center of the board to 150 Gauss in the farthest corner (dark blue) 


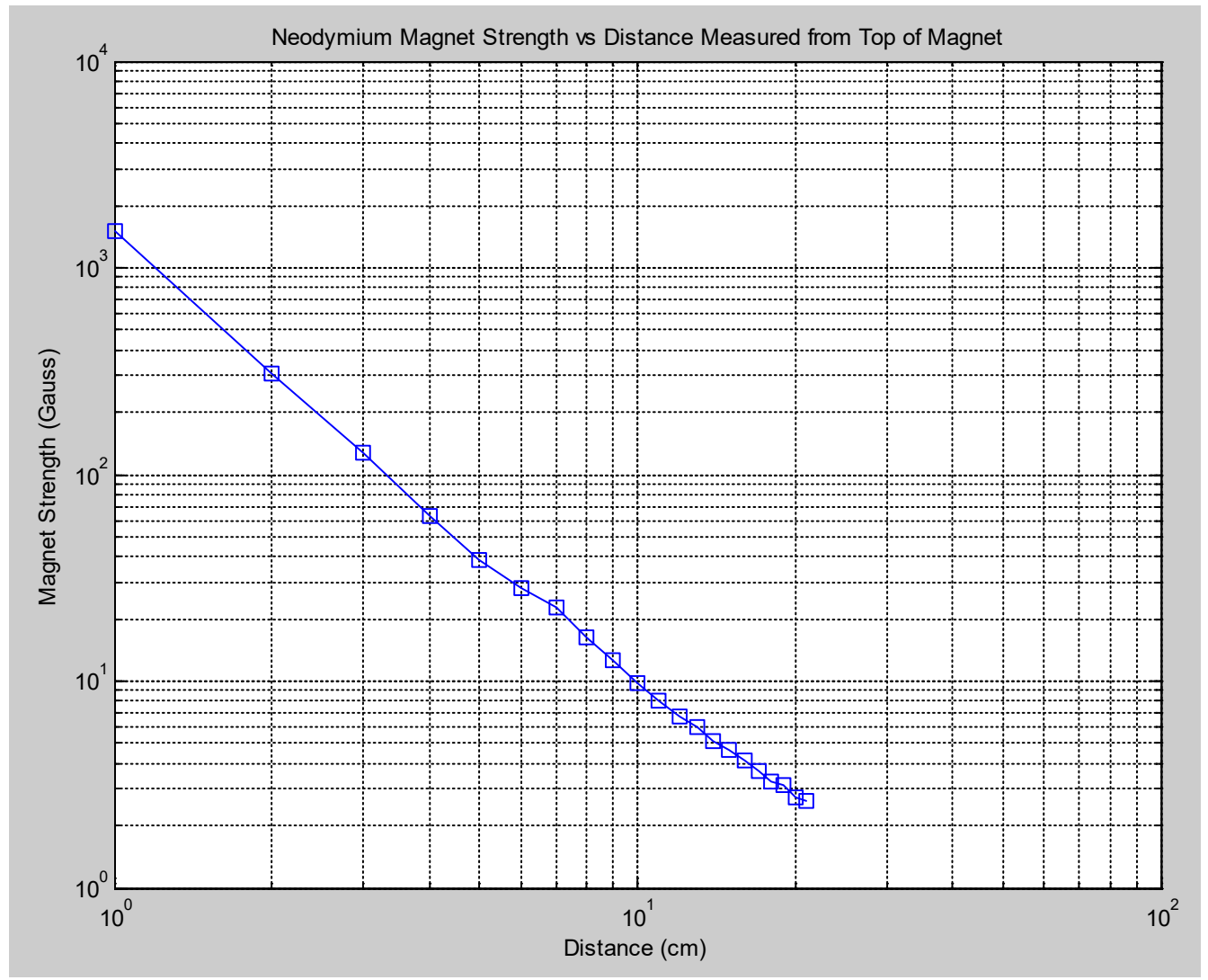

Figure 7: Magnetic field decay of a single hand soldering box magnet as a function of distance

\subsubsection{Reflow Oven}

The reflows ovens used for this experiment were designed and built by members of MRT for the purpose of creating a larger number of samples per parabola than is achievable by hand soldering alone. Additionally, there was some chance that the reflow oven may create superior samples compared to hand soldered joints as this is the intended melting method for solder pastes. The design uses two identical ovens to melt two boards worth of samples simultaneously for each parabola. One of these ovens has a magnet mounted to it so that one sample board per parabola can solidify in a magnetic field while the other solidifies undisturbed by the magnetic field. The magnetic oven was mounted to the top of 
the non-magnetic oven, as shown in Figure 7, to allow for both magnetic soldering and unmodified soldering on the same parabola, without using space in the payload allocated to other systems.

Each oven was an 8"x8"x8" outer aluminum shell, with 1" of SALI-2 insulation lining the interior, and one front panel attached to sliding rails that functioned as a door. The heating elements in these ovens were comprised of coils wound from 22-gauge 316 L stainless steel resistance wire with a total resistance of 18 Ohms. Operating at 115 VAC these heating elements draw up to $672 \mathrm{~W}$ each. To control these heating elements, a system of two PID controllers was used, with one PID set to maintain a soak temperature to preheat the board to just below the melt temperature and the other set well above the melt temperature, so that when this second PID is used during the parabolas the boards are continuously heating. A solid-state relay and toggle switch changed which PID was controlling the oven at any given time. This dual PID system was contained within a plastic electronics box mounted to the oven system's base plate, beneath the gloves of the operator; see Figure 8. 


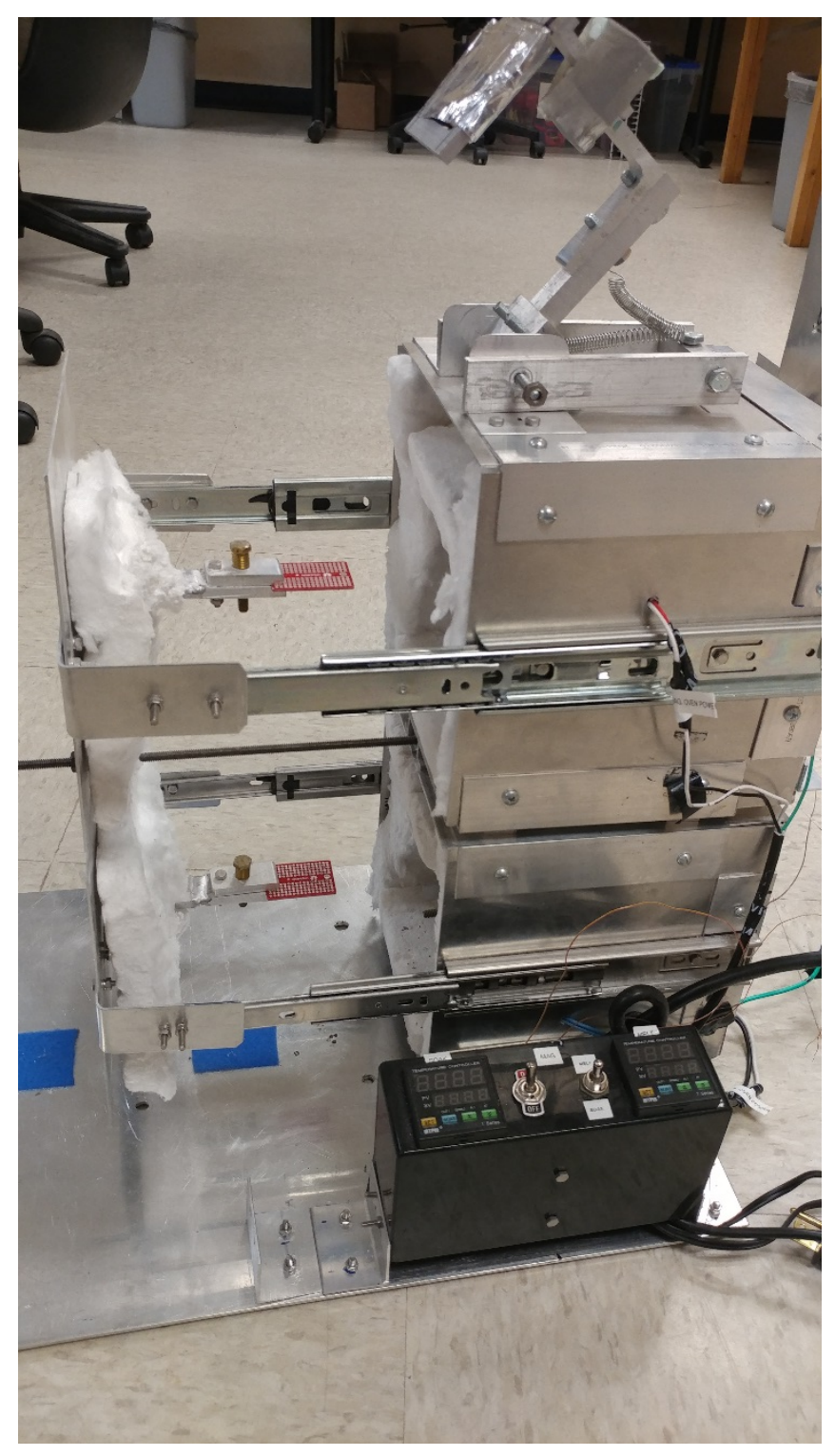

Figure 8: Reflow ovens

The sample boards for the oven were stored in a microscope slide box that was Velcroed to the polycarbonate enclosure panel next to the oven area. When in the ovens, the boards were held in place by a spring-loaded compression clip attached to the oven door. The paste-free end of the boards could be wedged into this clip and held securely during the heating and cooling processes. The 
placement of these clips was such that when the doors were closed, the boards would be positioned in the middle of, but not in contact with, the heating element. When the doors were open a magnet mounted on a hinged arm atop the upper oven would be folded down to create a magnetic field above the upper board while the solder samples cooled; see Figure 9.

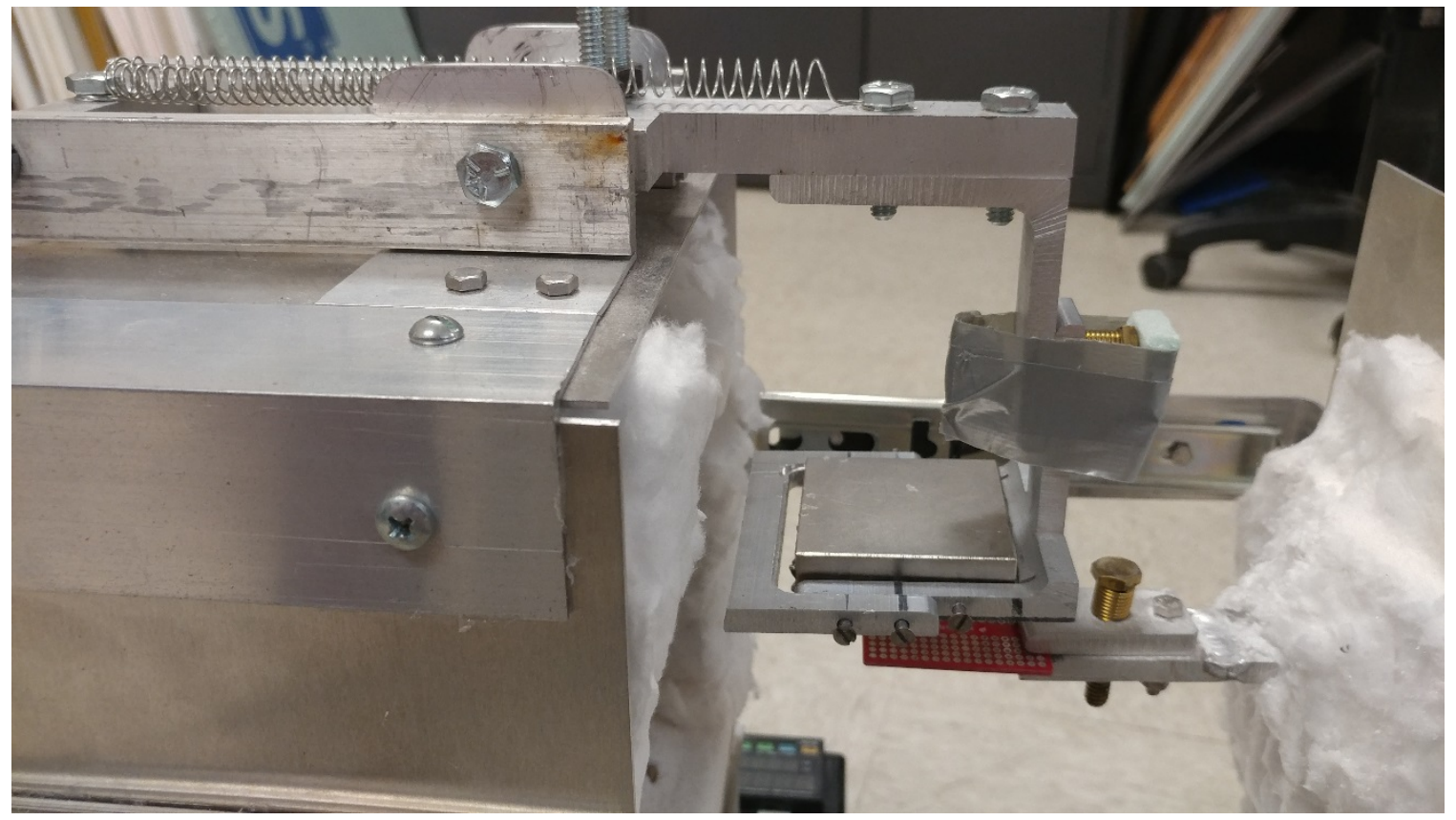

Figure 9: Placement of oven magnet above sample board when folding arm is deployed

\subsubsection{Induction}

Another group within MRT developed an induction heating system, based on a design from Carnegie Mellon [11], to melt solder using AC magnetic fields. The magnetic field was generated by passing an electrical current through a copper coil, using an oscillating board to feed this current to the coil switches the 
direction of the magnetic field and can heat ferrous objects inside the magnetic field.

This system used a 2" diameter, 2.5" long helical coil wrapped from 1/4" copper tubing powered by an $85 \mathrm{kHz}$ oscillating board. This coil was cooled using a recirculating water pump to prevent the coil from over-heating itself while running, which could change the electrical resistance of the coil and produce hysteresis effects for samples created during later parabolas.

To melt solder samples, a sample board was passed through the center of this coil, where the magnetic field is strongest. The sample boards also utilized a small piece of steel attached directly beneath the board to draw more energy from the magnetic field. The steel was attached to the board by folding the ends of the resistor's wires over the steel piece. The wires also served to transfer the heat of the steel to the solder paste.

The samples were moved axially through the helical coil by an Arduinocontrolled lead screw. This screw was placed below the coil and held samples at the level of the coil via an aluminum offset piece. The sample boards were attached to wooden extenders, which were clamped to the aluminum offset. This prevented any metal, other than the heating steel and resistor wires, from being directly placed in the stronger areas of the magnetic field, and lowered risk of them directly contacting the coil. The final induction heating system is shown in Figure 10 as it was for the ground testing, after being removed from the payload box. 


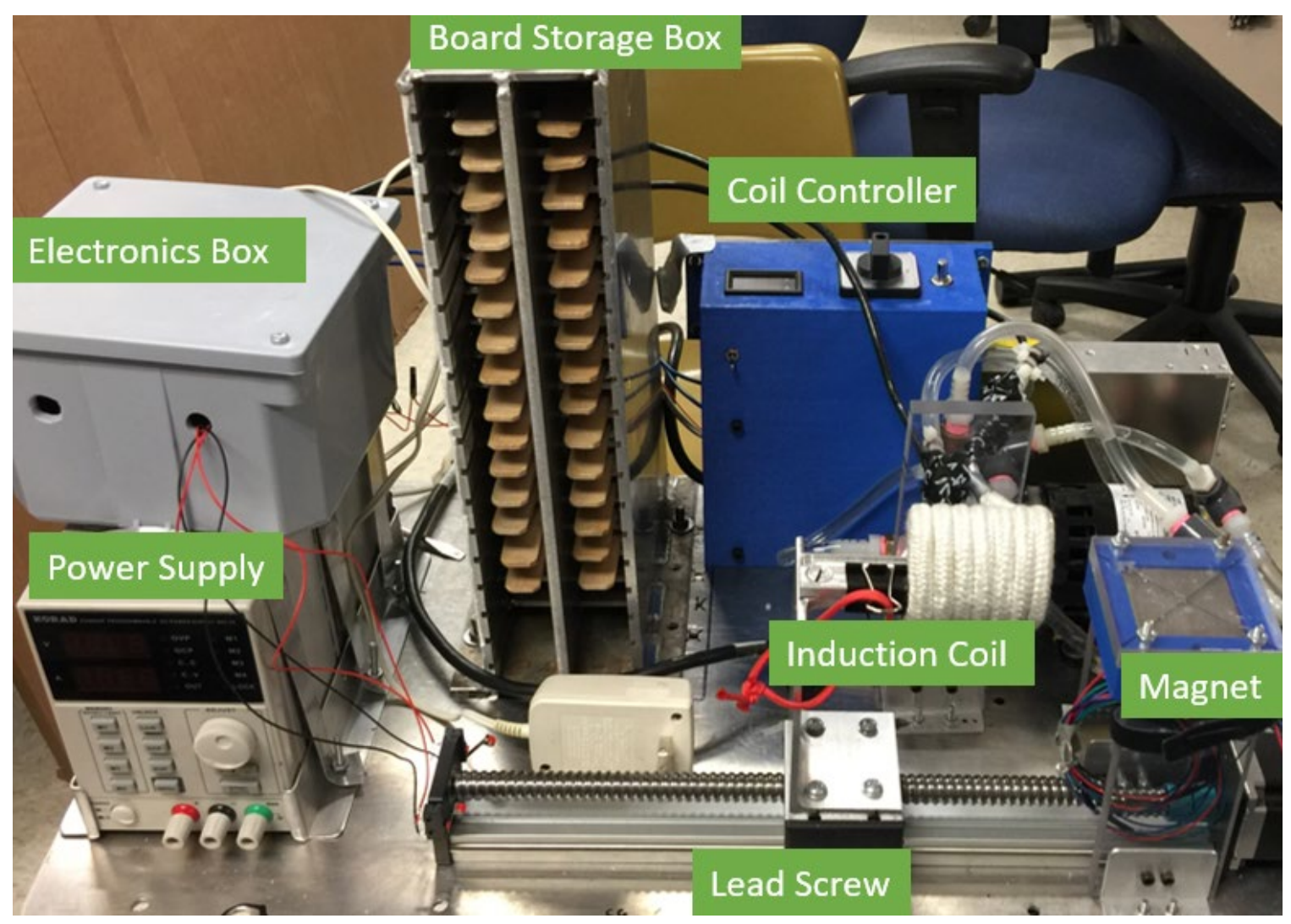

Figure 10: The induction heating system as configured for ground testing

Due to a leak in the cooling system during the flight safety review, the induction system was not used in flight, but was replaced with a fourth hand soldering station. Because of this, the only samples that were created by the induction system were ground testing samples. Although there was no flight data to compare these samples with, they were still processed and many of them analyzed along with the samples from the other soldering systems. 


\subsubsection{Flight Data Acquisition System}

A flight data acquisition system was mounted inside the payload during the flight that gathered acceleration data for the flight as well as environmental data for the inside of the payload box throughout the flight. An Adafruit data logger served as the controller for the system, which logged data from an accelerometer, a weather data sensor reporting temperature, humidity, and pressure data, and three additional temperature sensors spread throughout the payload. This system and the data it produced documented the conditions under which the solder joints were created.

\subsubsection{Payload Frame and Fume Containment}

The payload frame was constructed of 2.5 "x 2.5 " $\times 1 / 4$ " aircraft certified aluminum angle pieces bolted together with a pair of $1 / 4-28 \mathrm{NF}$, aircraft certified bolts at each joint. This frame was then bolted to a $1 / 2$ " aluminum mounting plate, as this was the minimum base thickness set by Zero-G. This design was calculated to be sufficient to survive $9 \mathrm{G}$ loading, as is required by Zero-G in case of a hard landing. For the purpose of securing components used inside the payload, a grid of $1 / 4 "$ holes was drilled into the baseplate at 6 " intervals. With this layout placements of systems or components could be changed without any additional changing to the payload baseplate. Also, components could be replaced more easily as each piece had the ability to be mounted at any location within the payload. 
The frame also was required to contain any fumes produced during soldering; for this reason, the top and sides of the payload frame had $1 / 4 "$ polycarbonate panels mounted to them. To allow access inside the box for soldering, holes were cut into the side and flanges mounted around these holes. Plastic gloves were then clamped to the outside of these flanges and fed into the payload to create a glovebox-like user interface. Inside the enclosed payload, a filtration system was installed to clear any smoke or fumes created by soldering. This filtration system consisted of a $240 \mathrm{cfm}$ duct fan with a series of three filters attached to it: two carbon filters and one HEPA filter. One carbon filter was mounted on the inlet side of the fan, and the HEPA filter and second carbon filter were mounted to the exit side. To ensure that the filtration system would filter all smoke or fumes created, all holes and seams in the payload were taped over to seal all gases inside, and the edges of the filters were taped to the side of the fan to ensure that all air flowing through the fan would be filtered. The complete experimental payload can be seen in Figure 11. 


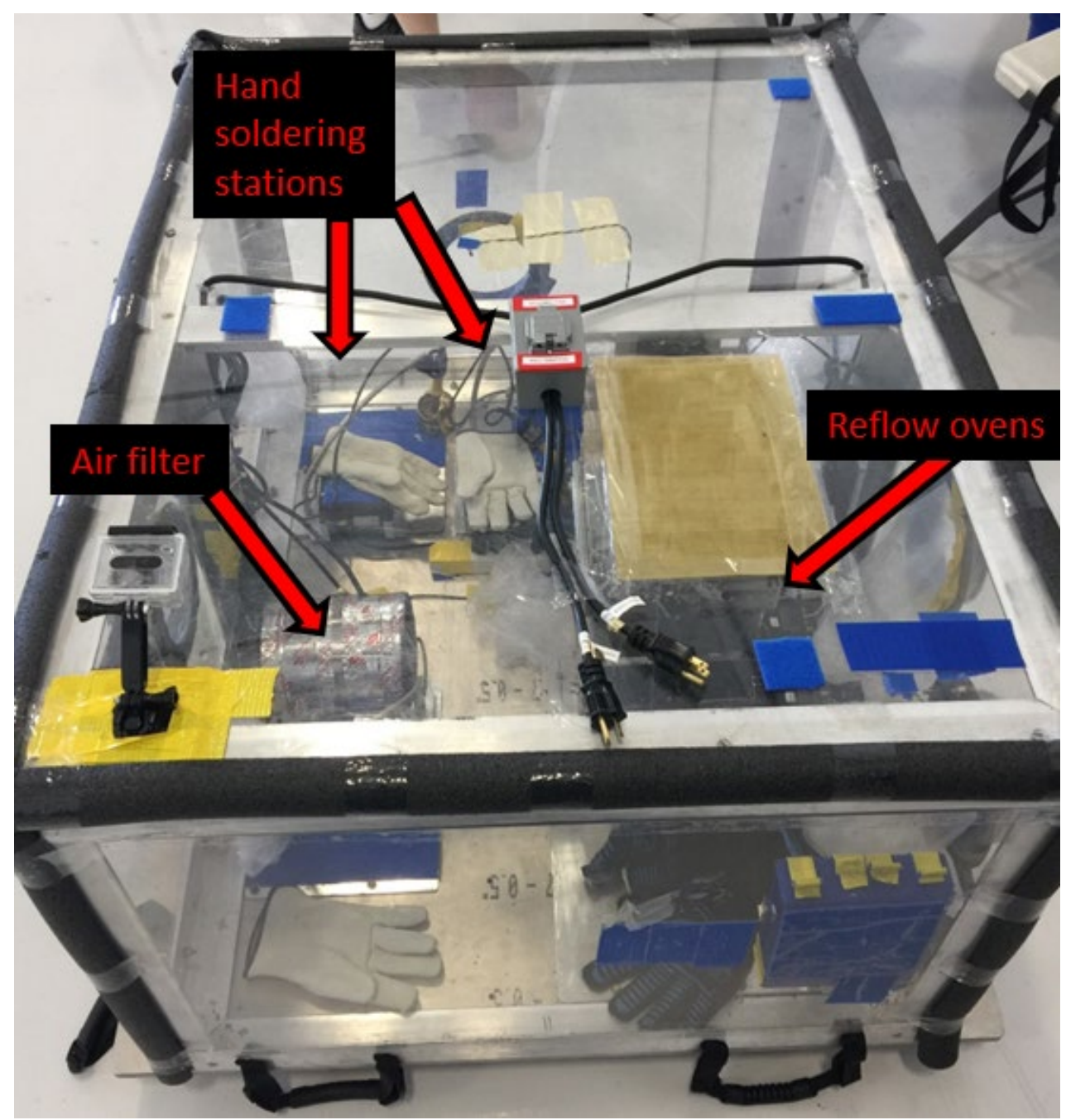

Figure 11: The payload assembly in flight configuration

\subsection{Flight Conditions and Procedures}

\subsubsection{Flight Plan}

The flight plan set by Zero-G began with takeoff from the Orlando Sanford International Airport in Sanford, FL and cruising at an altitude of $24,000 \mathrm{ft}$ to a designated airspace above the Atlantic Ocean. Once in the parabolic arc airspace, G-Force One climbed to $32,000 \mathrm{ft}$ and began a series of alternating parabolic arcs between $32,000 \mathrm{ft}$ and $24,000 \mathrm{ft}$. That provided periods of altered gravity levels on 
the plane switching between microgravity and hypergravity of nominally $1.8 \mathrm{G}$. The first five parabolas were not true microgravity, but rather reduced gravity; the first three parabolas give Martian gravity levels and the fourth and fifth, Lunar gravity levels. This gradual reduction in gravity level, eased the flyers into the microgravity environment. After the initial set of five parabolas, 25 microgravity parabolas were flown, in sets of five with short breaks in between. Each parabola provided approximately 20 seconds of microgravity with $1-1.5$ minutes between microgravity periods. Table 2 details the flight profile. After the $15^{\text {th }}$ parabola the plane turned around to fly the second half of the parabolas back toward the coast. Upon completion of the parabolas the plane return to the Sanford Airport, approximately one hour after departure. 
Table 2: Flight profile and timing

\begin{tabular}{|c|c|}
\hline \multicolumn{2}{|c|}{ Flight Profile } \\
\hline Parabola \# & G level/timing \\
\hline $\begin{array}{l}\text { Before parabolas } \\
\text { begin }\end{array}$ & 10 minutes - set up \\
\hline 1 & Martian \\
\hline 2 & Martian \\
\hline 3 & Lunar \\
\hline 4 & Lunar \\
\hline 5 & Lunar \\
\hline break & 3 minutes \\
\hline 6 & Zero \\
\hline 7 & Zero \\
\hline 8 & Zero \\
\hline 9 & Zero \\
\hline 10 & Zero \\
\hline break & 3 minutes \\
\hline 11 & Zero \\
\hline 12 & Zero \\
\hline 13 & Zero \\
\hline 14 & Zero \\
\hline 15 & Zero \\
\hline break & 3 minutes \\
\hline 16 & Zero \\
\hline 17 & Zero \\
\hline 18 & Zero \\
\hline 19 & Zero \\
\hline 20 & Zero \\
\hline break & 3 minutes \\
\hline 21 & Zero \\
\hline 22 & Zero \\
\hline 23 & Zero \\
\hline 24 & Zero \\
\hline 25 & Zero \\
\hline break & 3 minutes \\
\hline 26 & Zero \\
\hline 27 & Zero \\
\hline 28 & Zero \\
\hline 29 & Zero \\
\hline 30 & Zero \\
\hline After last parabola & $\begin{array}{l}10 \text { minutes - secure and } \\
\text { stow equipment }\end{array}$ \\
\hline
\end{tabular}




\subsubsection{Parabola Quality}

The DAQ system in the payload measured environmental data throughout the flight: temperature, pressure, humidity, and three-directional acceleration. These data points were sampled at a rate of $30 \mathrm{~Hz}$. The most important data in relation to properly testing the hypotheses of the experiment was the acceleration data. The DAQ recorded three axis acceleration data, one aligned with the aircraft's longitudinal axis, one aligned with the horizontal axis (wingspan axis), and one aligned with the vertical axis, the vertical axis being the acceleration that would be gravity in normal conditions. This is the only axis that undergoes major changes on the flight; both of the other axes have nearly zero acceleration throughout the flight, as they would on the ground. Figure 12 and Figure 13 show this variation in vertical acceleration as a percentage of sea-level gravity. The large fluctuations between nearly zero vertical acceleration to around 1.6 to 1.8 times Earth gravity seen in the plots correspond with the parabolas in the flight. The soldering was performed during the periods when these $\mathrm{G}$ levels bottom out, which can be better seen in Figure 13. 


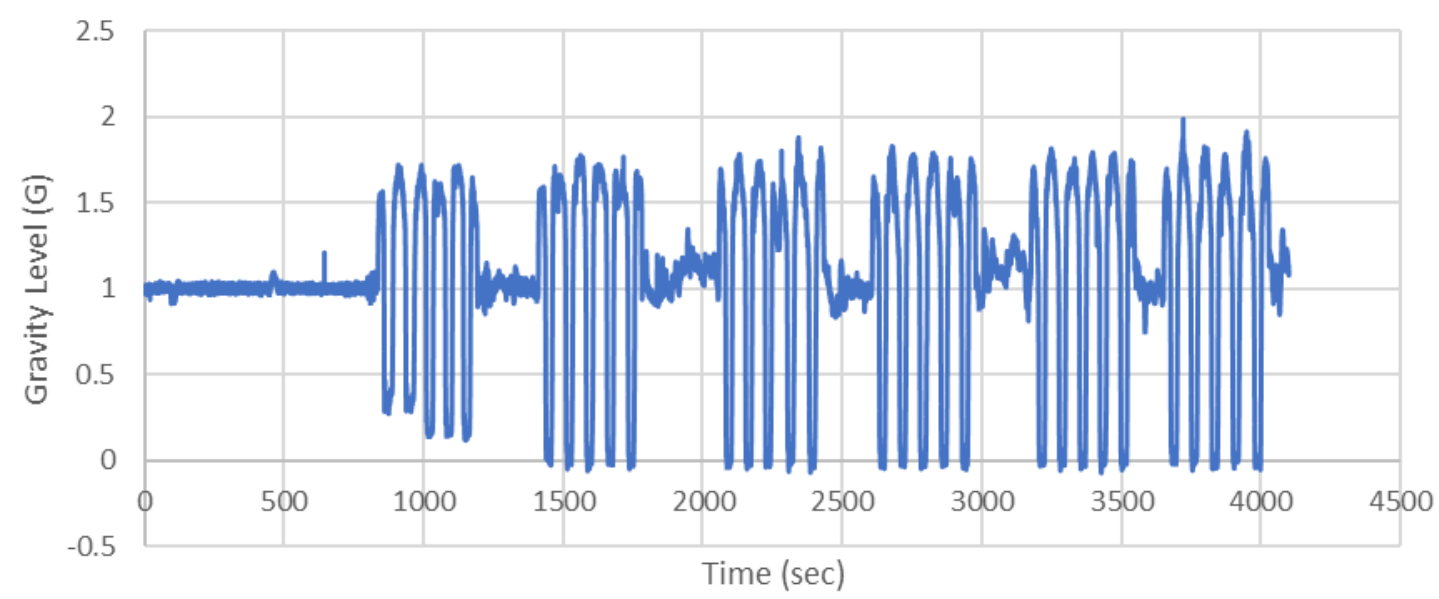

Figure 12: The vertical acceleration experienced by the payload

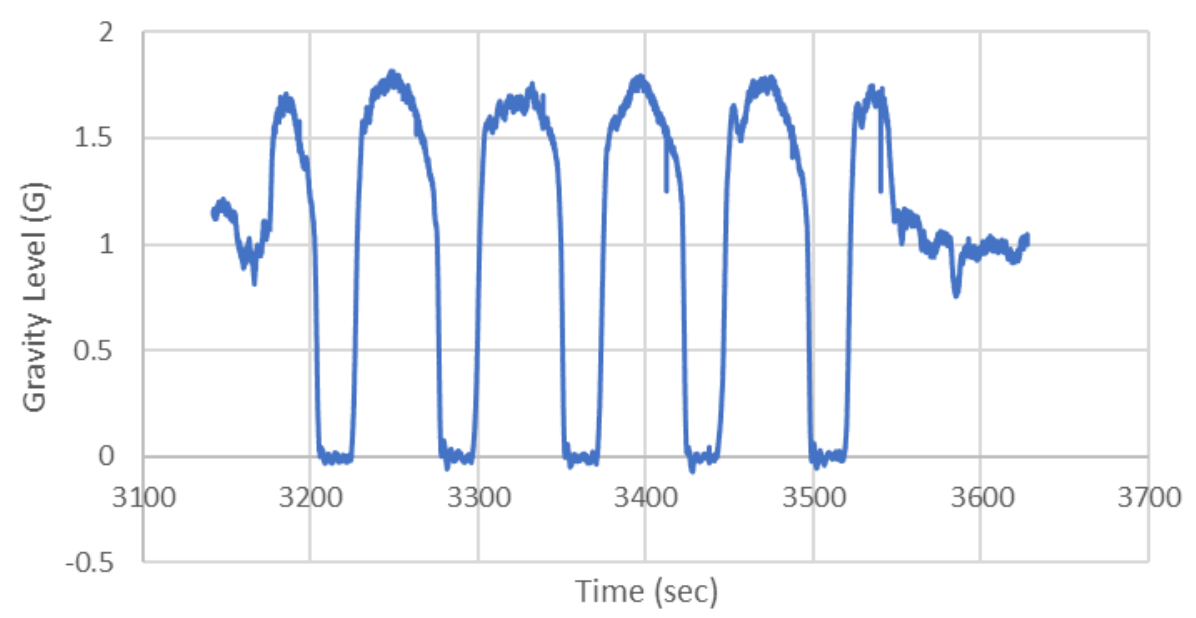

Figure 13: Gravity levels for one set of flight parabolas, parabolas 16 through 20

Along with general plots of gravity levels, the data was also reviewed to determine the statistical characteristics of each microgravity period (note that parabolas 1-5 were reduced gravity, not microgravity). To characterize the microgravity periods, what time interval constitutes microgravity must be defined. For the purpose of defining microgravity, three gravity levels were identified as possible cutoffs as the microgravity point: times when the acceleration was below 
$10 \% \mathrm{G}, 5 \% \mathrm{G}$, and $3 \% \mathrm{G}$, and the quality of the parabolas were analyzed. Based on the defined levels, the microgravity periods were evaluated by the average gravity level and gravity level RMS, for the period during each parabola that the gravity level was continuously below the defined level. The length of that period was also determined. Using this data, the quality of the microgravity period can be assessed; the average and RMS acceleration levels are presented in Figure 14 and Figure 15, respectively. The length of time spent in microgravity during each parabola is presented in Figure 16 with numerical values in Table 3. The data these tables were generated from can be found Appendix B: Parabolic Aircraft Gravity Data.

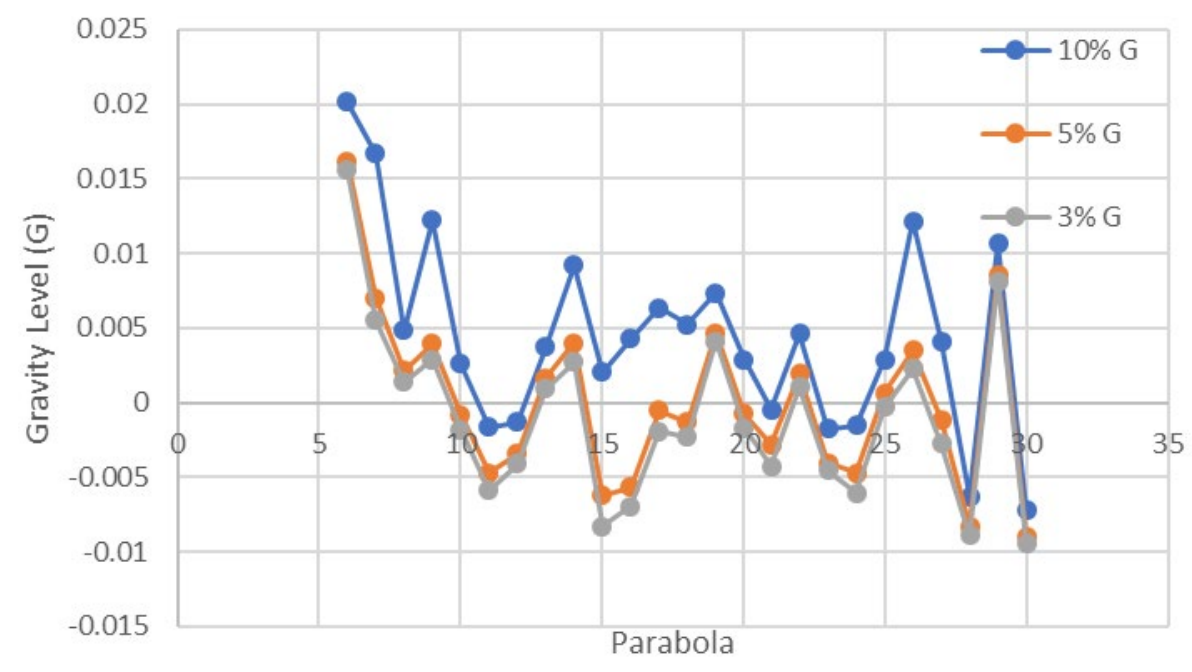

Figure 14: Average gravity level measured during each microgravity period 


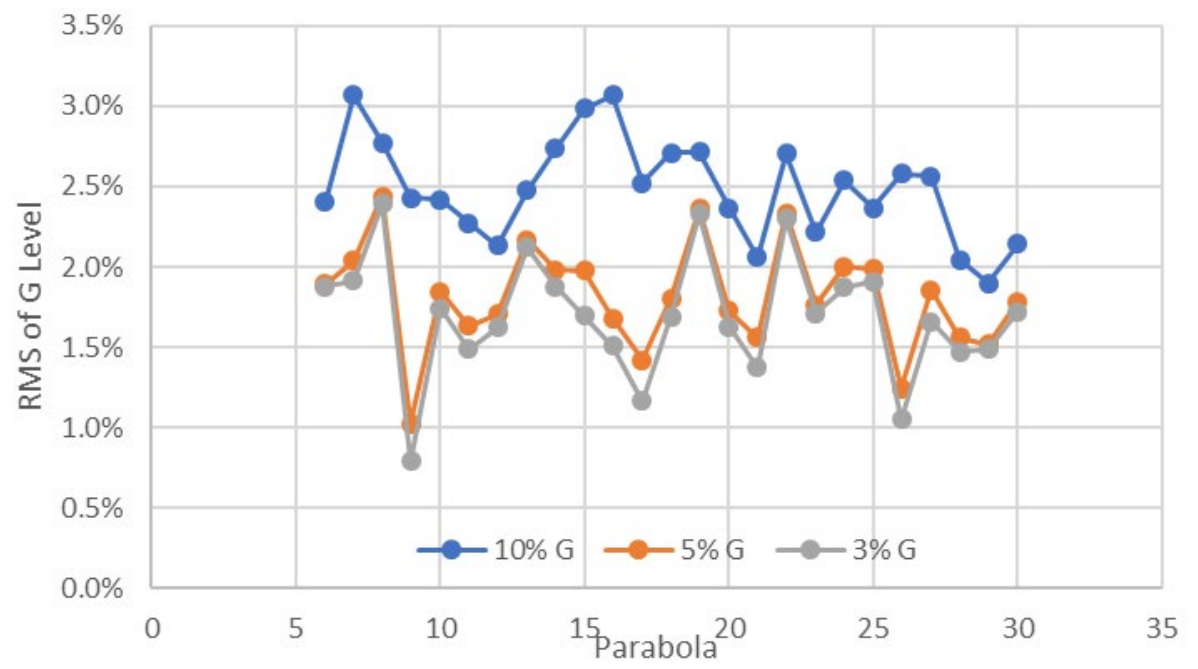

Figure 15: RMS of gravity level measurements during each microgravity period

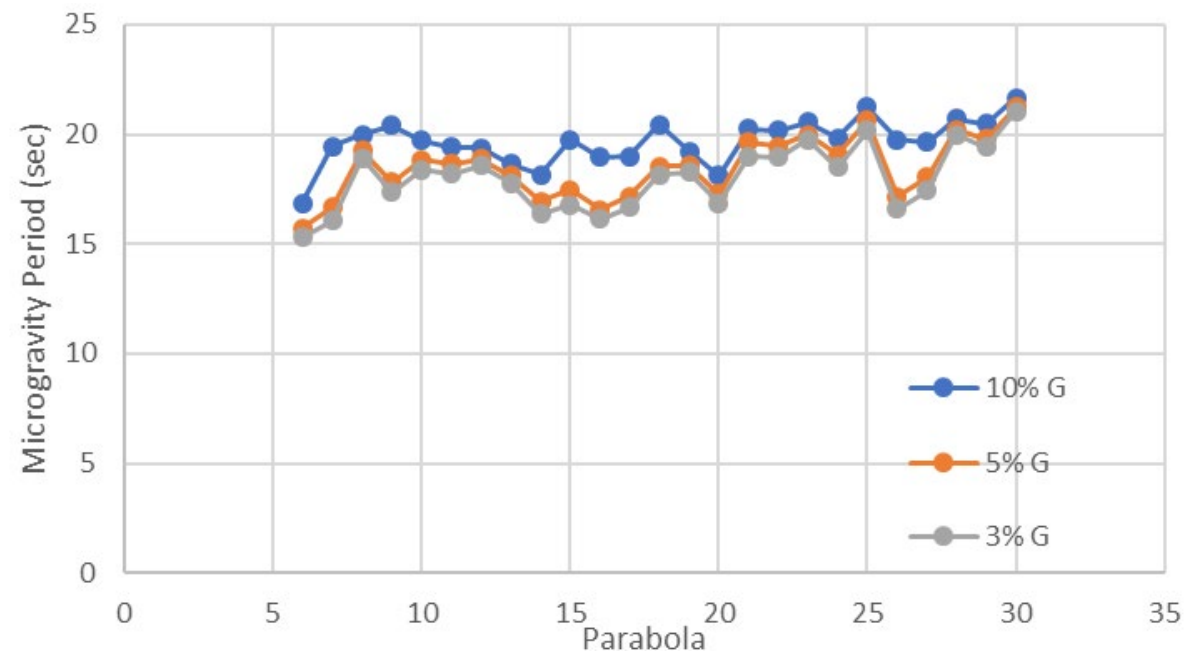

Figure 16: Length of microgravity periods in flight 
Table 3: Length of microgravity periods in flight

\begin{tabular}{|c|c|c|c|}
\hline \multicolumn{4}{|c|}{ Time in Microgravity } \\
\hline Parabola \#: & $10 \% \mathrm{G}$ & $5 \% \mathrm{G}$ & $3 \% \mathrm{G}$ \\
\hline 6 & 16.851 & 15.735 & 15.286 \\
\hline 7 & 19.46 & 16.675 & 16.073 \\
\hline 8 & 19.989 & 19.255 & 18.909 \\
\hline 9 & 20.388 & 17.825 & 17.345 \\
\hline 10 & 19.704 & 18.846 & 18.401 \\
\hline 11 & 19.395 & 18.659 & 18.18 \\
\hline 12 & 19.381 & 18.868 & 18.574 \\
\hline 13 & 18.635 & 18.119 & 17.766 \\
\hline 14 & 18.174 & 16.927 & 16.386 \\
\hline 15 & 19.763 & 17.457 & 16.788 \\
\hline 16 & 18.961 & 16.57 & 16.124 \\
\hline 17 & 18.987 & 17.17 & 16.691 \\
\hline 18 & 20.428 & 18.54 & 18.154 \\
\hline 19 & 19.237 & 18.565 & 18.278 \\
\hline 20 & 18.119 & 17.263 & 16.879 \\
\hline 21 & 20.241 & 19.657 & 18.975 \\
\hline 22 & 20.187 & 19.454 & 18.95 \\
\hline 23 & 20.558 & 19.985 & 19.761 \\
\hline 24 & 19.841 & 19.077 & 18.504 \\
\hline 25 & 21.246 & 20.637 & 20.16 \\
\hline 26 & 19.76 & 17.185 & 16.614 \\
\hline 27 & 19.628 & 18.043 & 17.439 \\
\hline 28 & 20.699 & 20.222 & 19.966 \\
\hline 29 & 20.486 & 19.786 & 19.413 \\
\hline 30 & 21.674 & 21.224 & 21.035 \\
\hline
\end{tabular}

Based on the data presented above, some observations can be made about the quality of the microgravity periods. All 25 parabolas had average acceleration levels that were within $0.02 \mathrm{G}$ of zero. After the tenth parabola, all but two parabolas averaged gravity within $0.01 \mathrm{G}$ of zero. Also, based on RMS levels, no parabolas were identified as having an excessive or abnormal amount of variation or G-jitter. Finally, the length of parabolas continually, but gradually extended throughout the 
duration of the flight, from about 15 seconds early in the flight seconds to slightly over 21 seconds near the end.

\subsubsection{Flyer Procedures}

Each flyer, or ground operator, had a standard process for sample generation that was repeated for every parabola. Other than initial setup and starting procedure at the beginning of the flight and shut down procedures at the end of the flight, all assigned tasks were repeated processes to be done every parabola or every break in parabolas.

Before the parabolas began, the experiment was made ready by powering on all equipment, and allowing heating elements to preheat to desired temperatures, then performing a test cycle of all equipment without soldering. The flyers then set up cameras throughout the payload to observe the experiment and bumped the DAQ system to sync the flight data with the cameras. As final step before beginning the parabolas, the flyers opened sample boxes to make the boards accessible.

Once the parabolas began, the flyers began the procedures for generating sample solder joints. Hand solderers began soldering at the onset of microgravity, beginning at the top left of the first sample board in their sample boxes and moved right soldering as many joints as could complete in that row, up to four possible joints; then the iron tips were cleaned. On subsequent parabolas solderers would 
move to the next row down the board and repeat this process. During breaks between parabolas, they were instructed to clean their tips thoroughly and adjust the temperature setting on the irons. The temperature started the first set of parabolas at $290 \mathrm{C}$ during the flight and $250 \mathrm{C}$ on the ground and increased by $20 \mathrm{C}$ at every break. For ground testing the third break had a larger increase, by $60 \mathrm{C}$ to $350 \mathrm{C}$, which matches the flight temperature at that time.

The reflow oven operator was responsible for the use of both ovens onboard. This flyer's soldering process required the heaviest workload and began before the microgravity period. During hypergravity, the designated pair of samples for the parabola, were moved from the sample storage container to the board clips in the ovens and the ovens were set to a holding temperature, or 'soak temperature', below the solder's melting point. Approximately 5 to 10 seconds before the microgravity period began, based on the operator's judgement, the oven operator would flip a switch on the control boxes to increase the oven temperature in order to melt the solder. Then 10 seconds into the microgravity period the oven was opened to cool the boards for the remainder of the microgravity period. At this point a magnet above the upper oven was moved into position over the top board to stimulate flow in the molten magnetic solder and motion of the vapor bubbles. The sample boards were then moved to a holding box and the next set of boards placed in the oven and this process repeated for each parabola. 
The induction heating system was the most automated of the solder melting systems; in that all operations, except for exchanging samples, were controlled or initiated by buttons and computer control. This flyer would start the heating coil and the linear actuator with a button, then, once the sample was in place above the magnet, the coil was powered off and the actuator returned to its original position. The samples were then placed back in the holding box and a new sample placed for the next parabola. As with the reflow ovens, it was necessary for the heating process to begin slightly before entering microgravity, and again this timing was left to the judgement of the operator.

After the final parabola, all equipment was shut off and the power to the payload cut was off, sample boxes were then again closed, and the cameras stopped and stowed for landing. Once on the ground the sample boxes were removed from the payload to prevent any damage to them during unloading and transportation of the payload.

\subsection{Data Acquisition and Reduction}

\subsubsection{Sample Processing}

After generating samples during the flight and ground tests, the boards were cleaned. As the solder paste melts, flux flows from the joint and can create a layer of solidified flux residue on top of and around the solder joints. In some cases, this flux layer can incorporate particles of unmelted paste to create an opaque layer that prevents the joint from being accurately assessed. Some solder fluxes are 
acidic, so if flux is left on the board it could erode the surface of the board or the joints on it. For these reasons, the sample boards were cleaned as part of the postflight processing. An example of a sample box prior to cleaning of the boards can be seen in Figure 17 with Figure 18 showing a closer image of an uncleaned board. The dull gray material covering some of the joints is the residue from unmelted paste, and the yellow/brown areas around joints are the result of flux flowing onto the boards from the joints.

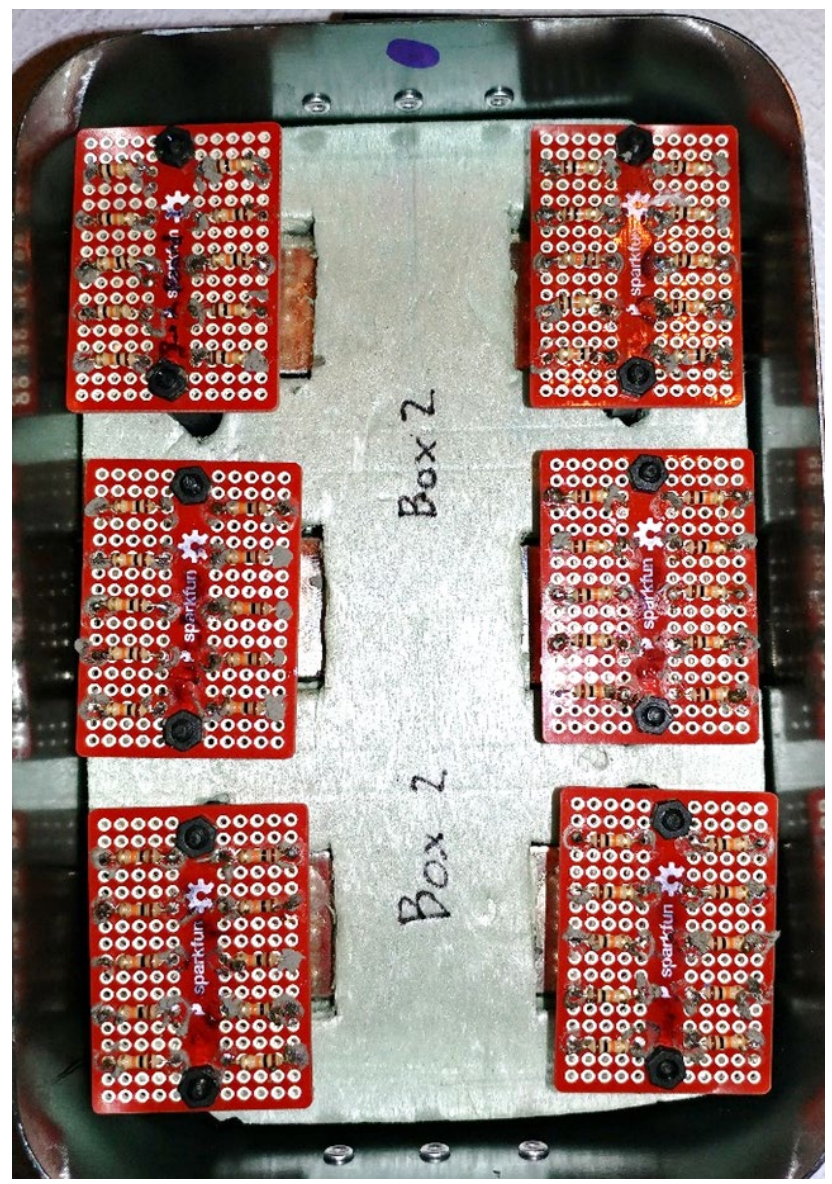

Figure 17: A hand-soldering sample box after flight, prior to board cleaning 


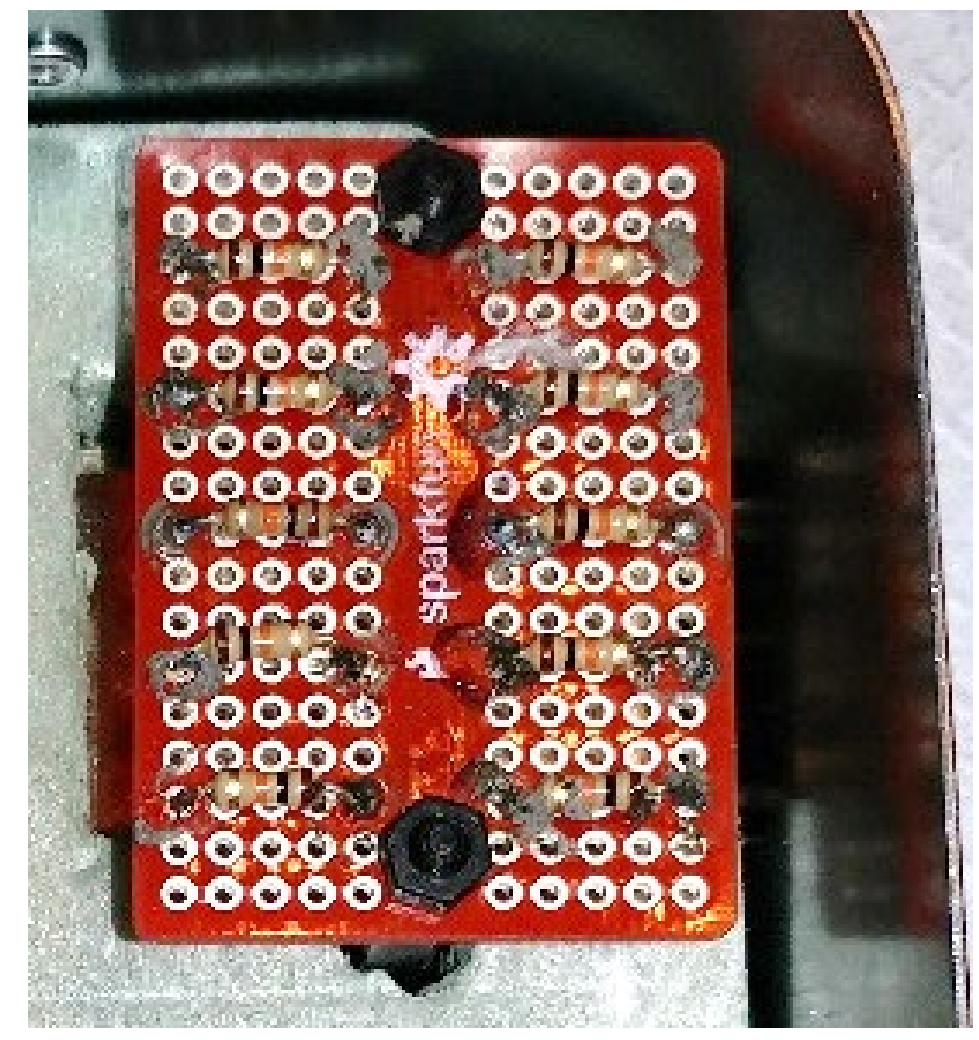

Figure 18: A hand soldering board before cleaning

To clean the boards, they were first soaked in an alcohol-based flux remover compound for a period of 5-10 minutes, then scrubbed with a plastic brush to remove residual flux and any solder that was not melted during testing. After the boards were cleaned, a visual inspection was performed on the solder joints; the details of this inspection are presented in the Visual Analysis section below.

Once the visual inspection was complete, each board had its resistors clipped off and was cut into sections as illustrated in Figure 19, so that each joint could be cross-sectioned for interior voidage analysis. The board was cut using a Dremel cutting tool so that each row of solder joints was its own piece of board, 
then these pieces were cut down the middle, so that they could be put into labeled sample jars and later would more easily fit into SEM sample holders for analysis. To keep the pieces organized during the cutting process each board had its left side (when viewed from the top) marked with black ink. Then, as the pieces were cut off of the main section of boards, the hand soldered sections were separated by what parabola the piece corresponds to, while the reflow oven samples had their edges marked in different colors of ink to indicate their positions on the board. For the oven samples the colored pieces were placed in the same sample jar, but the hand soldering samples were keep separated, this way both sets of joints could easily be identified by the parabola during which the joints were made.

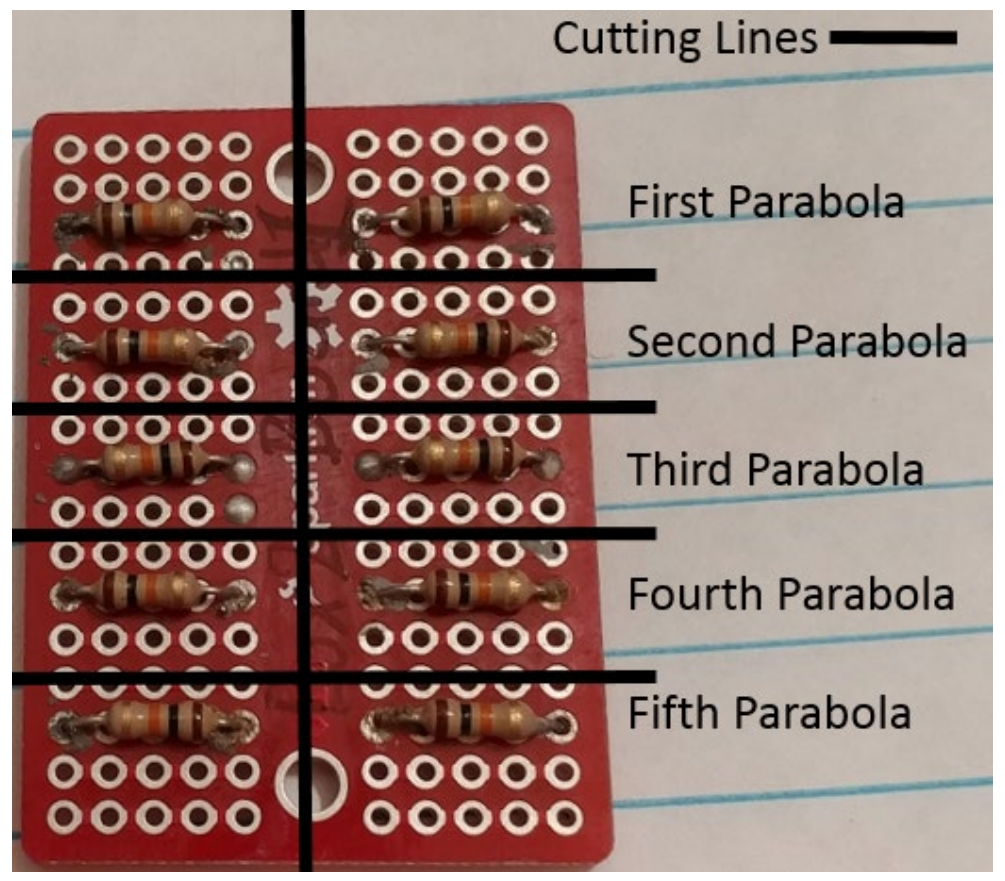

Figure 19: Illustration of cutting lines on a hand soldering sample board 
Once the boards were cut into sections, they were manually sanded and polished to create a flat cross-sectional surface. Starting with the cut sections, the boards were sanded to the edge of the through holes containing the solder joints using 120 grit sandpaper. Once the edges of the through holes were exposed, the joints themselves were sanded down to nearly the center of the joint using progressively finer sandpapers, up to 1500 grit, as the exposed surface neared the center. After reaching the desired location in the joint, the samples were polished, first with 3000 grit paper, then 5000 grit, and eventually 7000 grit to remove any scratching that occurred during the coarser sanding. Once the joints were thoroughly polished, they had a sufficiently flat surface that the majority of disturbances remaining in the surface were the voids in the solder.

\subsubsection{Visual Analysis}

The first assessment of the solder joints took place once the solder joints had been returned to WVU's campus after the flight. This analysis took the form of a visual inspection for exterior quality of the hand soldered joints. The joints were graded using a visual inspection guide [24] and each joint given a grade 0-3; with 0 being a failed joint or no joint attempted, 1 for joints with joints with visible defects, 2 for acceptable joints, and 3 for good joints which met the standard.

These evaluation results are in Appendix C: Hand Soldering Visual Inspection. As these joints were inspected, the ones that were found to be defective had their defects recorded to allow for comparing the types of defects 
seen in flight and on ground. Figure 20 and Figure 21 show examples of the visual inspection; these figures display the grades a box of hand soldered joints received (Figure 20) and the reasons some were marked as defective (Figure 21). In total, 11 types of defects were identified in the hand soldered joints created through both flight and ground testing, Table 4 lists each of these defect types as well as a brief description of their meaning.

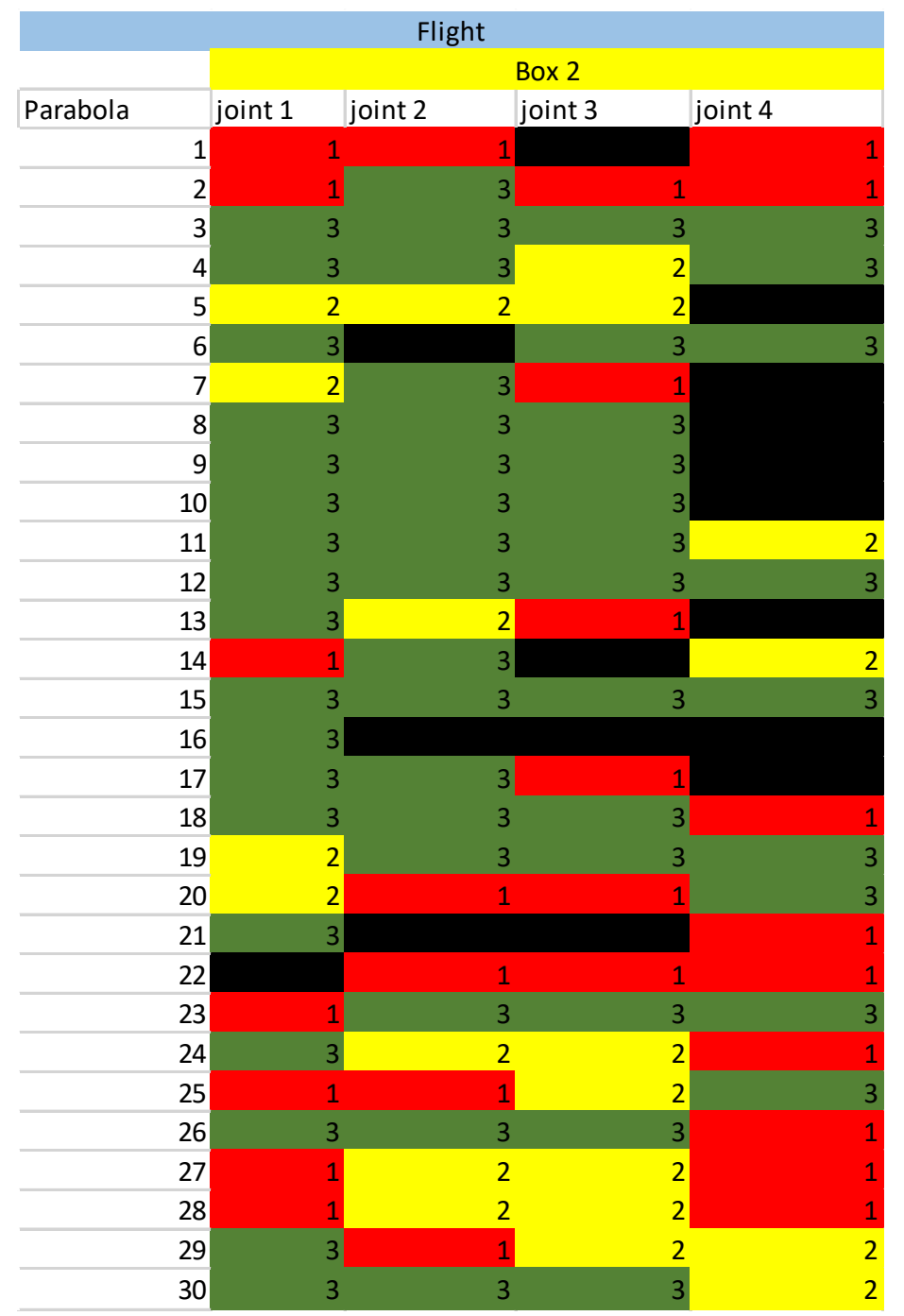

Figure 20: An example of the grading of joints in a sample box 


\begin{tabular}{|c|c|c|c|c|c|c|c|}
\hline \multicolumn{8}{|c|}{ Flight } \\
\hline \multirow[b]{2}{*}{ Parabola } & \multicolumn{7}{|c|}{ Box 2} \\
\hline & \multicolumn{2}{|c|}{ joint 1} & \multicolumn{2}{|c|}{ joint 2} & \multicolumn{2}{|c|}{ joint 3} & \multirow{2}{*}{$\begin{array}{l}\text { joint } 4 \\
\text { fill }\end{array}$} \\
\hline 1 & fill & & fill & & & & \\
\hline 2 & fill & & & & fill & & fill \\
\hline 3 & & & & & & & \\
\hline 4 & & & & & & & \\
\hline 5 & & & & & & & \\
\hline 6 & & & & & & & \\
\hline 7 & & & & & fill & & \\
\hline 8 & & & & & & & \\
\hline 9 & & & & & & & \\
\hline 10 & & & & & & & \\
\hline 11 & & & & & & & \\
\hline 12 & & & & & & & \\
\hline 13 & & & & & fill & & \\
\hline 14 & contact angle & & & & & & \\
\hline 15 & & & & & & & \\
\hline 16 & & & & & & & \\
\hline 17 & & & & & wet angle & wetting & \\
\hline 18 & & & & & & & surface void \\
\hline 19 & & & & & & & \\
\hline 20 & & & fill & & wet angle & & \\
\hline 21 & & & & & & & surface void \\
\hline 22 & & & fill & contact angle & & & fill \\
\hline 23 & surface void & & & & & & \\
\hline 24 & & & & & & & surface void \\
\hline 25 & & & & & & & \\
\hline 26 & & & & & & & \\
\hline 27 & fill & & & & & & fill \\
\hline 28 & through hole & wet angle & & & & & fill \\
\hline 29 & & & through hole & wet angle & & & \\
\hline 30 & & & & & & & \\
\hline
\end{tabular}

Figure 21: An example log of defects showing what errors were present in joints marked defective 
Table 4: Hand soldering defects observed in samples and their description

\begin{tabular}{|l|l|}
\hline \multicolumn{1}{|c|}{ Defect } & \multicolumn{1}{c|}{ Defects and their Descriptions } \\
\hline fill & The joint did not fill the entirety of the through hole \\
\hline contact angle & Solder balled on the surface \\
\hline projection & Spike like feature on the surface of the solder joint \\
\hline bridged holes & The solder flow into an adjacent hole as well as the intended hole \\
\hline surface void & The joints surface had a cavity \\
\hline wet angle & An area of the through hole or surface pad was not wetted by the solder \\
\hline wetting & Solder did not wet the wire and/or through hole \\
\hline through hole & The joint had a continuous hole through the entire joint \\
\hline climbed wire & Solder wicked up the wire further than is acceptable \\
\hline wrong hole & Solder melted into a hole without a resistor \\
\hline surface spread & Solder spread on the board beyond the soldering pad \\
\hline
\end{tabular}

\subsubsection{Microscopy}

Each sample was imaged using the 18 MP CCD through an Omax compound microscope in the microgravity lab once they were cross-sectioned and polished. For this imaging, a 4x objective was used in the microscope, which, when combined with the 10x eyepiece magnification, results in a total magnification of 40x. At this magnification, most of the solder joints could have the entire joint in the viewing area.

Some joints with a larger area, due to an excess of paste used or spreading on the wires, were too large to be photographed in a single frame. For these samples, multiple images were taken, allowing the images to be combined into a single view of the entire joint. Other joints exhibited uneven polishing, and therefore also needed multiple images with focal adjustments between images. On uneven surfaces, the entire surface cannot be in focus at the same time, so by taking 
multiple images of these joints, and adjusting the microscope's focus between images, each area of the joint can be photographed in focus and these areas combined for void observation.

Some samples were selected to be further analyzed by scanning electron microscopy (SEM) and energy dispersive x-ray spectroscopy (EDS). Using SEM allows the samples to be inspected at higher magnifications, which was used on samples that had interesting features such as large voids wide enough to allow the bottom of the void to be imaged. The use of SEM is also necessary for EDS in order to determine atomic species present in images, as the EDS imager is an add-on to the SEM hardware. A pair of images of a cross-sectioned joint is shown in Figure 22. It should be noted that the scratches from polishing the samples can be seen in the optical microscope's image (left) and in many cases the scratches are more apparent than this.

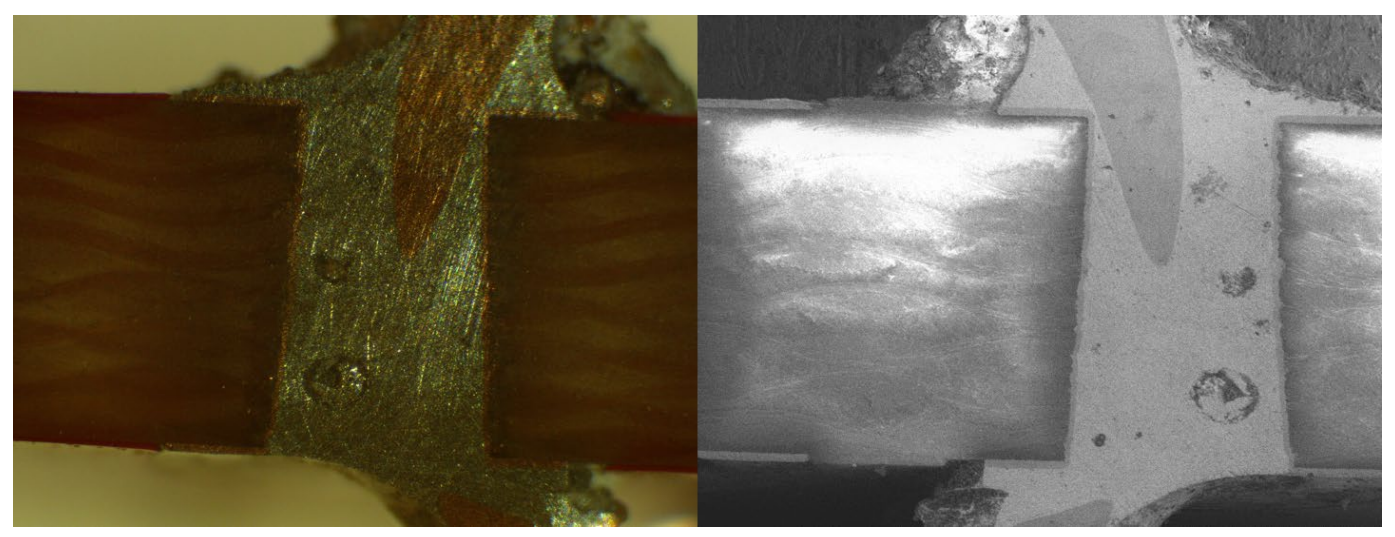

Figure 22: Microscope images of a solder joint. An optical microscope image (left) and an SEM image (right) both at $40 \mathrm{x}$ 
Energy dispersive x-ray spectroscopy analysis was used for a limited portion of the samples generated. During scanning electron microscopy, an EDS system can be used to determine atomistic species present within the area of a sample viewed by the SEM. This technology was used both to find iron particle locations within the solder for the purpose of determining the alignment of these particles in relations to each other and their proximity to locations of void, and also to attempt to gain insight into the sources of the voids found in the solder joints created.

The effects of iron were observed via EDS, primarily looking at the relationship between the iron dispersoids and voids in joints. Previous researchers $[19,20,17]$ have indicated that the presence of a dispersoid could cause an impedance to vapor flow through the molten solder. For this reason, the crosssectioned solder joints were analyzed to determine if areas around voids had higher concentrations of iron particles than areas with fewer voids.

EDS was also used to analyze the species of atoms on the interior surface of voids, to compare these areas of the joint cross-sections with areas that were comprised of void-free solder. It is expected that the interior surfaces of the voids contain higher concentrations of carbon and oxygen, which are the major components of the organic fluxes found in the solders used for this experiment. Therefore, finding higher carbon and oxygen in voids within the solder would 
indicate that the voids were created by trapping vaporized flux within the molten solder, as previous researchers have speculated. $[7,8,9,10]$

\subsubsection{Image Processing}

Microscopy images of solder joints were digitally processed to calculate the percent voidage. Using Adobe Photoshop, the images were edited to remove everything except the solder joint from in the images by creating a mask layer that covered all other areas of the image in black. This mask was created by tracing the area to be blacked out to create a border and using a fill function to color the area black. By creating this layer, the blacked-out areas can be transferred between images of the same joint, so that only in-focus areas are being used for the creation of the masking layer. This ensures that all areas of the joint are viewed at the same magnification and preserves details that would be lost if out of the focal area.

Once all non-solder areas of the images were blacked out, including wires through the joint and the samples board, the voids within the joint were identified and these areas were colored red in a similar process to selecting the blacked out area and on the same layer as the mask. This left the planar surface of the joint as the only uncolored area. Upon exporting this layer from Photoshop, any uncolored areas, representing the solder, were converted to white in the final image. This resulted in tricolor images that clearly identified the areas designated as solder (white), void (red), and background (black). Figure 23 gives an example of what a 
tricolor image looks like based on the polished cross- section used to create it, and more of these side-by-side images with porosity data can be seen in Appendix D: Visual Comparison of Microscope Images vs Processed Images with Voidage Percentages.

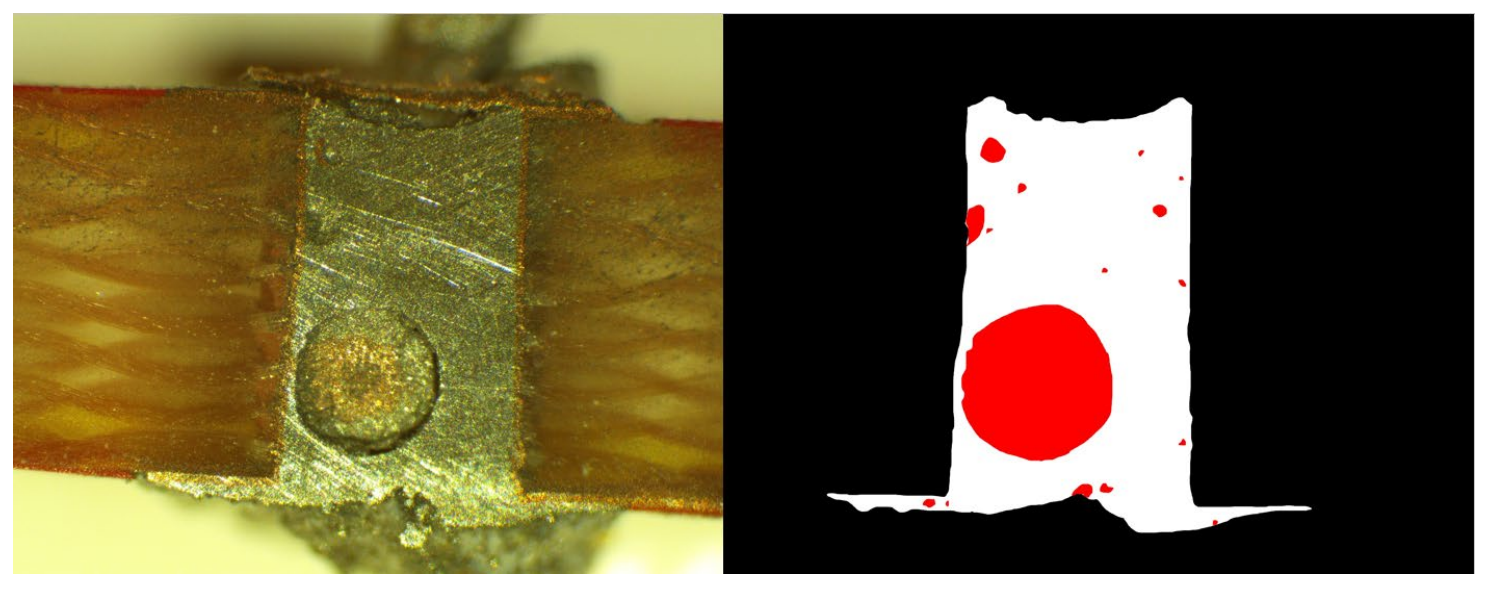

Figure 23: A microscope image and its tricolor counterpart

Using the tricolor images, a MATLAB script was written to calculate the percent voidage of the joints. The script identifies the white and red colored areas and, based on the ratio of areas that were identified as being voids in the tricolor images to the areas of void and planar solder (red/[red+white]), outputs a percent voidage for each tricolor image which indicates the voidage percent of that joints cross section. The output from this image processing is then compiled into tables like Table 5, which were used to calculate the results presented in 4.3 Interior Joint Porosity, these can be found in Appendix E: Sample Porosity. 
Table 5: Solder joint porosity

\begin{tabular}{|c|c|c|c|c|c|c|c|c|c|c|c|c|c|}
\hline \multicolumn{14}{|c|}{ Flight } \\
\hline \multicolumn{14}{|c|}{ Box 1} \\
\hline Parabola & Joint & & Colorization 1 & Colorization 2 & Colorization 3 & Average & Parabola & Joint & & Colorization 1 & Colorization 2 & Colorization 3 & Average \\
\hline \multirow[t]{4}{*}{1} & & 1 & & & & & 16 & & 1 & & & & \\
\hline & & 2 & & & & & & & 2 & & & & \\
\hline & & 3 & & & & & & & 3 & & & & \\
\hline & & 4 & & & & & & & 4 & & & & \\
\hline \multirow[t]{4}{*}{2} & & 1 & & & & & 17 & & 1 & & & & \\
\hline & & 2 & & & & & & & 2 & 1.3278 & & & 1.3278 \\
\hline & & 3 & & & & & & & 3 & & & & \\
\hline & & 4 & & & & & & & 4 & & & & \\
\hline \multirow[t]{4}{*}{3} & & 1 & & & & & 18 & & 1 & 2.032 & & & 2.032 \\
\hline & & 2 & & & & & & & 2 & & & & \\
\hline & & 3 & & & & & & & 3 & & & & \\
\hline & & 4 & & & & & & & 4 & & & & \\
\hline \multirow[t]{4}{*}{4} & & 1 & & & & & 19 & & 1 & 2.4946 & & & 2.4946 \\
\hline & & 2 & & & & & & & 2 & 1.2923 & & & 1.2923 \\
\hline & & 3 & & & & & & & 3 & & & & \\
\hline & & 4 & & & & & & & 4 & & & & \\
\hline \multirow[t]{4}{*}{5} & & 1 & & & & & 20 & & 1 & & & & \\
\hline & & 2 & & & & & & & 2 & & & & \\
\hline & & 3 & & & & & & & 3 & & & & \\
\hline & & 4 & & & & & & & 4 & & & & \\
\hline \multirow[t]{4}{*}{6} & & 1 & 3.5313 & 5.164 & & 4.34765 & 21 & & 1 & & & & \\
\hline & & 2 & 3.5813 & & & 3.5813 & & & 2 & 13.2706 & & & 13.2706 \\
\hline & & 3 & 2.1961 & & & 2.1961 & & & 3 & & & & \\
\hline & & 4 & 1.5612 & & & $\quad 1.5612$ & & & 4 & & & & \\
\hline \multirow[t]{4}{*}{7} & & 1 & 5.1635 & & & 5.1635 & 22 & & 1 & 1.2923 & & & 1.2923 \\
\hline & & 2 & 21.2104 & & & 21.2104 & & & 2 & & & & \\
\hline & & 3 & 1.8366 & & & 1.8366 & & & 3 & & & & \\
\hline & & 4 & & & & & & & 4 & & & & \\
\hline \multirow[t]{4}{*}{8} & & 1 & 8.5343 & & & 8.5343 & 23 & & 1 & 5.4788 & & & 5.4788 \\
\hline & & 2 & 1.9547 & & & 1.9547 & & & 2 & & & & \\
\hline & & 3 & 5.7641 & & & 5.7641 & & & 3 & & & & \\
\hline & & 4 & & & & & & & 4 & & & & \\
\hline \multirow[t]{4}{*}{9} & & 1 & 8.7643 & 7.007 & & 7.88565 & 24 & & 1 & & & & \\
\hline & & 2 & 2.8121 & 4.26 & & 3.53605 & & & 2 & 14.7379 & & & 14.7379 \\
\hline & & 3 & 4.6027 & 5.175 & & 4.88885 & & & 3 & & & & \\
\hline & & 4 & & & & & & & 4 & & & & \\
\hline 10 & & 1 & 3.9281 & 6.929 & & 5.42855 & 25 & & 1 & & & & \\
\hline & & 2 & 29.2792 & 35.432 & & 32.3556 & & & 2 & & & & \\
\hline & & 3 & & & & & & & 3 & & & & \\
\hline & & 4 & & & & & & & 4 & & & & \\
\hline 11 & & 1 . & 2.9526 & 9.853 & 6.701 & 6.5022 & 26 & & 1 & & & & \\
\hline & & 2 & 3.8926 & 11.355 & 4.897 & 6.714867 & & & 2 & & & & \\
\hline & & 3 & 4.8025 & 17.042 & & 10.92225 & & & 3 & & & & \\
\hline & & 4 & & & & & & & 4 & & & & \\
\hline 12 & & 1 & & & & & 27 & & 1 & & & & \\
\hline & & 2 & & & & & & & 2 & & & & \\
\hline & & 3 & & & & & & & 3 & & & & \\
\hline & & 4 & & & & & & & 4 & & & & \\
\hline 13 & & 1 & 1.0204 & & & 1.0204 & 28 & & 1 & & & & \\
\hline & & 2 & 2.7277 & & & 2.7277 & & & 2 & 1.3215 & & & 1.3215 \\
\hline & & 3 & & & & & & & 3 & & & & \\
\hline & & 4 & & & & & & & 4 & 2.0468 & & & 2.0468 \\
\hline 14 & & 1 & 1.5159 & & & 1.5159 & 29 & & 1 & & & & \\
\hline & & 2 & 1.027 & & & 1.027 & & & 2 & 3.0573 & 5.463 & & 4.26015 \\
\hline & & 3 & & & & & & & 3 & & & & \\
\hline & & 4 & & & & & & & 4 & & & & \\
\hline 15 & & 1 & 2.9773 & & & 2.9773 & 30 & & 1 & 2.3601 & & & 2.3601 \\
\hline & & 2 & & & & & & & 2 & & & & \\
\hline & & 3 & 1.0497 & & & 1.0497 & & & 3 & & & & \\
\hline & & 4 & & & & & & & 4 & & & & \\
\hline
\end{tabular}




\section{Results}

\subsection{Vision Inspection (First Round Qualitative Analysis)}

The first evaluation of the solder joints was a visual inspection of the exterior of the joints after the flight. This inspection included only those joints created by hand soldering. There were 595 joints ( 279 ground and 316 flight) out of a potential 840 that would have been the max possible based on the box setups, however when excluding the first 5 parabolas of the flight this reduces to 539 total joints and 260 in flight. Since the inspection criteria were based on the quality of attachment of an electrical component to a circuit board, the samples created by the reflow ovens, which were only paste flowed in holes in boards and did not attach any component, were not considered during this analysis. Nor were the samples created by the induction heating system as these samples also had no component connections and because there was no possible data comparison as this system was not flown in microgravity.

During the visual analysis, samples were rated as: good, acceptable, or defective. Samples made under varying conditions were compared based on the percentage of the samples of each of the solder compositions that were rated into each grade. In total there were six variables that were adjusted for the samples: gravity level, iron content of the solder paste, the base paste used, the presence of magnetic fields, the iron temperature, and the individual operating the soldering station. 
The variation of the iron temperature was of the least importance, as it had similar results for all conditions. As the iron temperature increased, the number of joints found in visual inspection to be good increased and the number of defective joints decreased. However, the increased quality was only seen in visual inspection of the joints and temperature had no consistent effect on the interior voidage of the joints. Due to the consistency of the effect of temperature change, illustrated in Figure 24, it will not be further considered as a variable affecting other conditions.

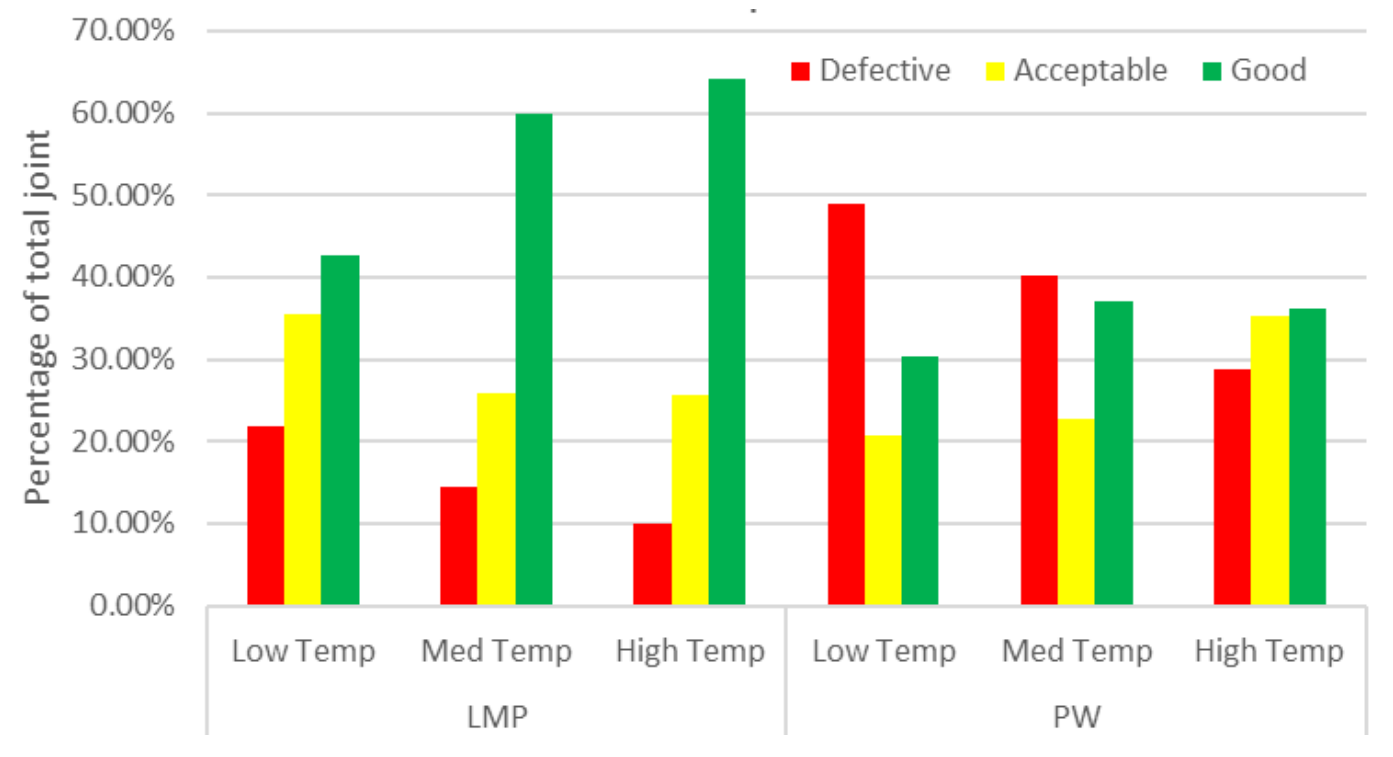

Figure 24: Effects of soldering iron temperature on quality of joints

Another source of variance between samples present, although not inherently by design, was the solderer using a sample box. Only two of the hand solderers were present during both the ground test and the flight, therefore, any 
direct comparison between fight and ground for an individual must be focused on these solderers. Solderer 1 used sample boxes without magnets during both flight and ground testing, while Solderer 2 had a magnet box during the flight testing but not during the ground testing. The results of the comparison of flight and ground visual inspection data for these two solderers, Figure 25 and Figure 26, indicate that based on exterior quality of joints the number of joints created changed between the two tests. When the data is normalized by the total number of joints made, the percentage of joints falling into each quality grade was similar, with both solderers having a slight increase in quality during the flight, but with Solderer 2 improving slightly more between the two tests, so that these two solderers had nearly identical percentages of defective joints during the microgravity flight.

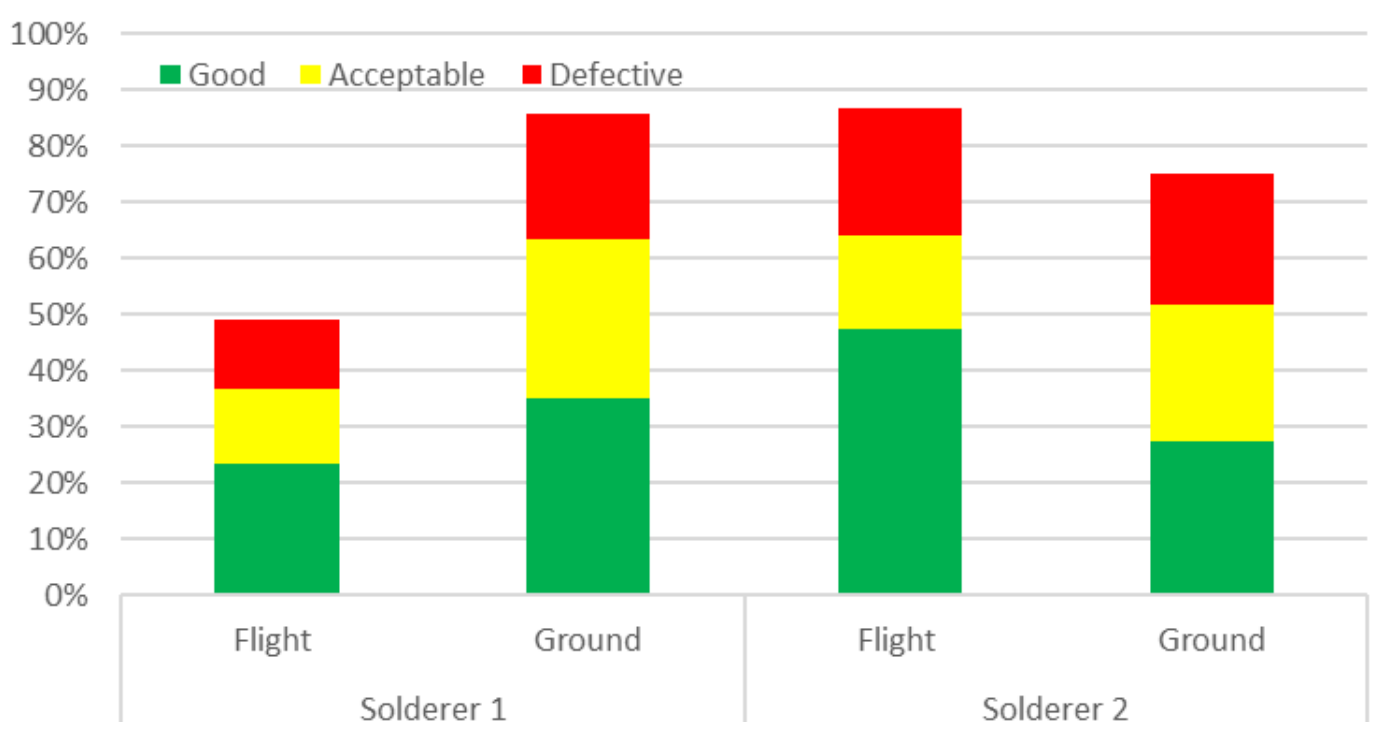

Figure 25: Quality of joints created by solderers on ground and in flight as percentage of total possible joints in each grade 


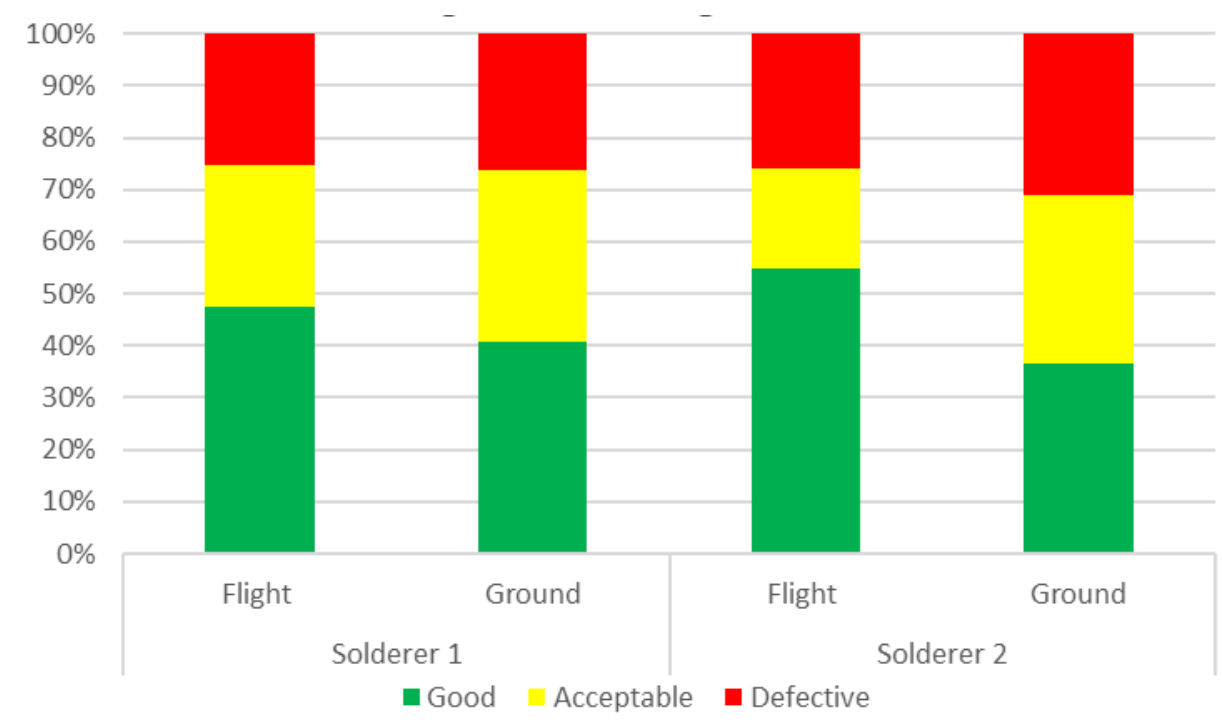

Figure 26: Quality of joints comparing flight and ground for solderers as percentage of joints they produced

The drop in the number of samples created by Solderer 1 during the flight was explained by the solderer in a post-flight briefing to be a result of being more cautious of soldering outside the microgravity times, being sure to not begin soldering before the beginning of microgravity and not soldering any joints that may still be molten when the microgravity period would end.

The rise in number of samples created by Solderer 2 , as well as the slight increase in percent of joints rated as 'good' for both solderers on the flight may be explained, at least in part, as a result of increased familiarity with the equipment. The ground test, the first of the two tests, was the first time either of these operators soldered inside the payload box. It is possible that as they became accustomed to the conditions imposed on them by the payload: restricted range of motion, 
separation from their soldering target, and using gloves while soldering, the joints they created would be of improved quality and be produced at a higher rate.

Although that is the only direct comparison that can be made how solderers performed between the two tests, but an overall evaluation can be made of the solderers' performances. By looking at the quality of joints created by each solderer, it is possible to measure how well they performed during the experiment. The metric used in this determination was the ratio of good joints created per defective joint, or good/defective ratio. Table 6 quantifies the performance of the solderers of each sample box as well as a comparison of the two solderers who were present for both tests, denoted as Solderers 1 and 2, who respectively soldered flight box 1 and ground box 1 , and flight box 2 and ground box 3 .

Table 6: Evaluation of solderer performance

\begin{tabular}{|c||r|r|}
\hline \multirow{2}{*}{ Conditions } & Samples & \multicolumn{1}{|l|}{$\begin{array}{l}\text { Good to } \\
\text { Defective } \\
\text { Ratio }\end{array}$} \\
\hline \multirow{2}{*}{ Flight } & Box 1 & 1.867 \\
\hline \multirow{2}{*}{ Ground } & Box 2 & 2.111 \\
\cline { 2 - 3 } & Box 3 & 2.059 \\
\hline & Box 4 & 0.818 \\
\hline Repeat Solderers & Box 2 & 1.556 \\
\cline { 2 - 3 } Overall Ratio & Solder 1 & 3.571 \\
\cline { 2 - 3 } & Solder 2 & 1.179 \\
\hline
\end{tabular}


From the data presented in Table 6, there are two noticeable outliers for soldering quality, these being ground box 2 which had a significantly higher good/defective ratio than any other box and flight box 4 , which is much lower than the others and the only box to contain more defective joints than good joints. Ground box 2 was the only box on the ground to use magnets, so it is unknown whether the improvement was due to the quality of the solderer or due to that box having a larger force exerted on the molten solder with both the gravity and magnetic forces combining.

Flight box 4's lower good/defective ratio may be explainable by events that occurred during the flight, the student who was posted to that station became ill during the first set of parabolas and had to be removed from the experiment area. Because of this, the majority of the joints created in that box were soldered by a 'flight coach', a Zero-G employee paired with the team to oversee the onboard activities and provide guidance in the microgravity environment. This coach lacked the familiarity with the experiment that the other solderers had which may have contributed to the poorer quality of joints. Although these two sample boxes are distinct from the others, neither of these outliers have a serious impact on the overall trends of the data.

Eliminating the operator and iron temperature as variables leaves: solder paste, iron content, gravity level, and magnetic field as the remaining variables during analysis. Table 7 breaks down all the sample joints created to show how 
many fit in each category based on combinations of these variables, composition and environment. These samples are further separated in Table 8 to show the results of the visual inspection, dividing each category into the three possible quality grades.

Table 7: Number of solder samples in each category

\begin{tabular}{|c|c|c|c|c|c|c|c|c|}
\hline \multirow{5}{*}{ Flight } & \multicolumn{4}{|c|}{ Magnet } & \multicolumn{4}{|c|}{ No magnet } \\
\hline & & $0 \%$ & $4 \%$ & $6 \%$ & & $0 \%$ & $4 \%$ & $6 \%$ \\
\hline & LMP & 19 & 30 & 26 & LMP & 6 & 11 & 9 \\
\hline & PW & 29 & 57 & 52 & PW & 5 & 9 & 7 \\
\hline & Total & 48 & 87 & 78 & Total & 11 & 20 & 16 \\
\hline \multirow{5}{*}{ Ground } & \multicolumn{4}{|c|}{ Magnet } & \multicolumn{4}{|c|}{ No magnet } \\
\hline & & $0 \%$ & $4 \%$ & $6 \%$ & & $0 \%$ & $4 \%$ & $6 \%$ \\
\hline & LMP & 17 & 11 & 19 & LMP & 37 & 34 & 30 \\
\hline & PW & 18 & 13 & 12 & PW & 39 & 26 & 23 \\
\hline & Total & 35 & 24 & 31 & Total & 76 & 60 & 53 \\
\hline
\end{tabular}


Table 8: Number of solder samples in each category, sorted by visual quality

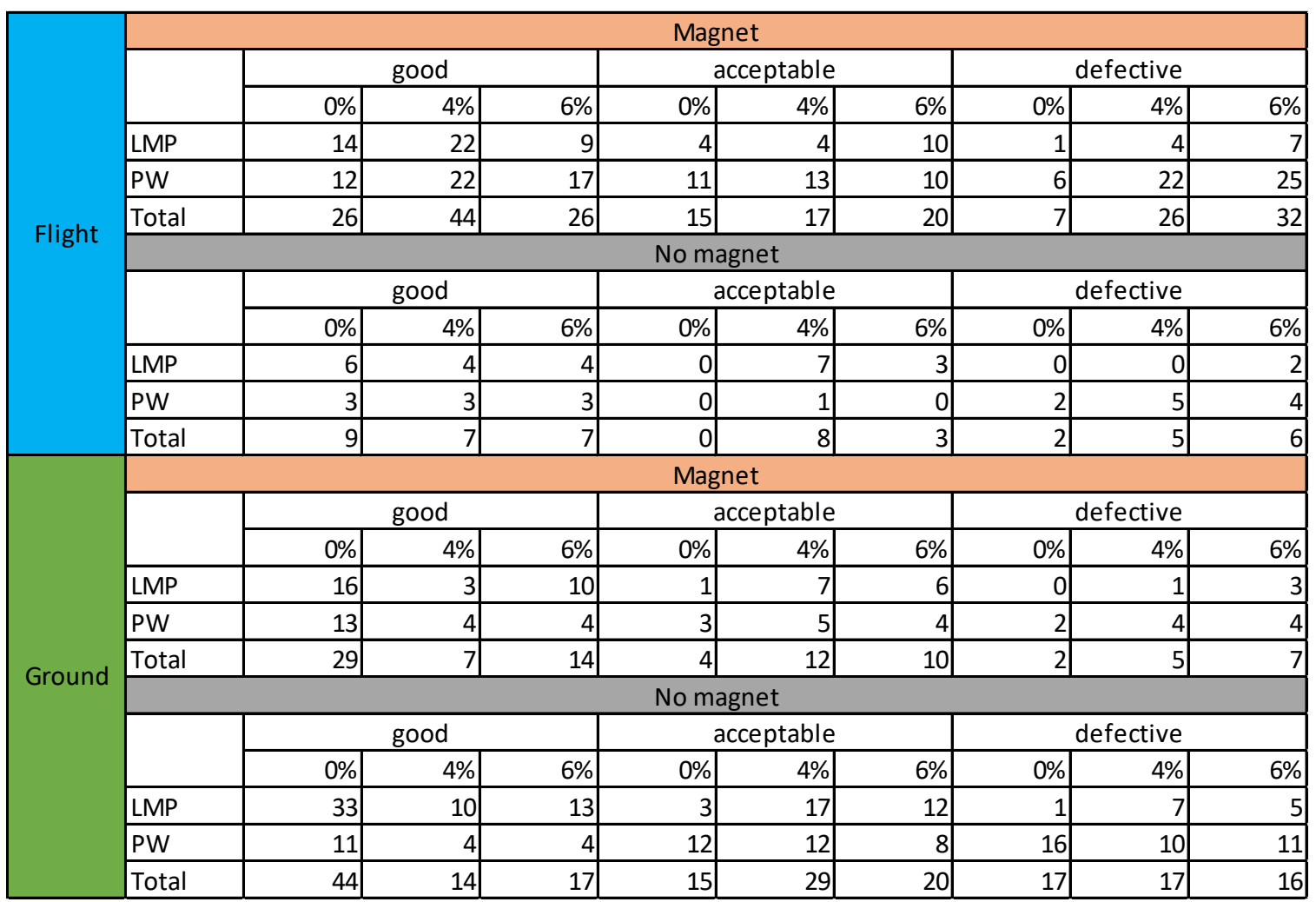

Using the sample breakdown in Table 8, comparisons between the categories show the effect each variable had on the quality of the solder joints. However, as seen previously the number of joints that fall into each category varies. Rather than basing judgement on total numbers, it would be more accurate to use the ratio of joints of each quality to the overall number of joints.

Table 9 shows how the data looks once normalized by the total number of joints created with each set of variables. From this table it seems that the LMP solder consistently out-performed the PW solder for all iron percentages. In each case the solder with $6 \%$ iron had the largest percentage of defective joints and in 
all but one instance, $4 \%$ showed a higher defect percentage than not having iron at all. This may be based on the dispersoid making the solder more viscous and less likely to properly flow fully into holes. This change was noticed even before the soldering began, as the paste was being applied to the sample boards, pushing the paste out of the syringe became more difficult when changing from $0 \%$ to $4 \%$ and was extremely difficult to apply to the boards at $6 \%$.

Table 9: Percentage of joints from each category that fall into each quality

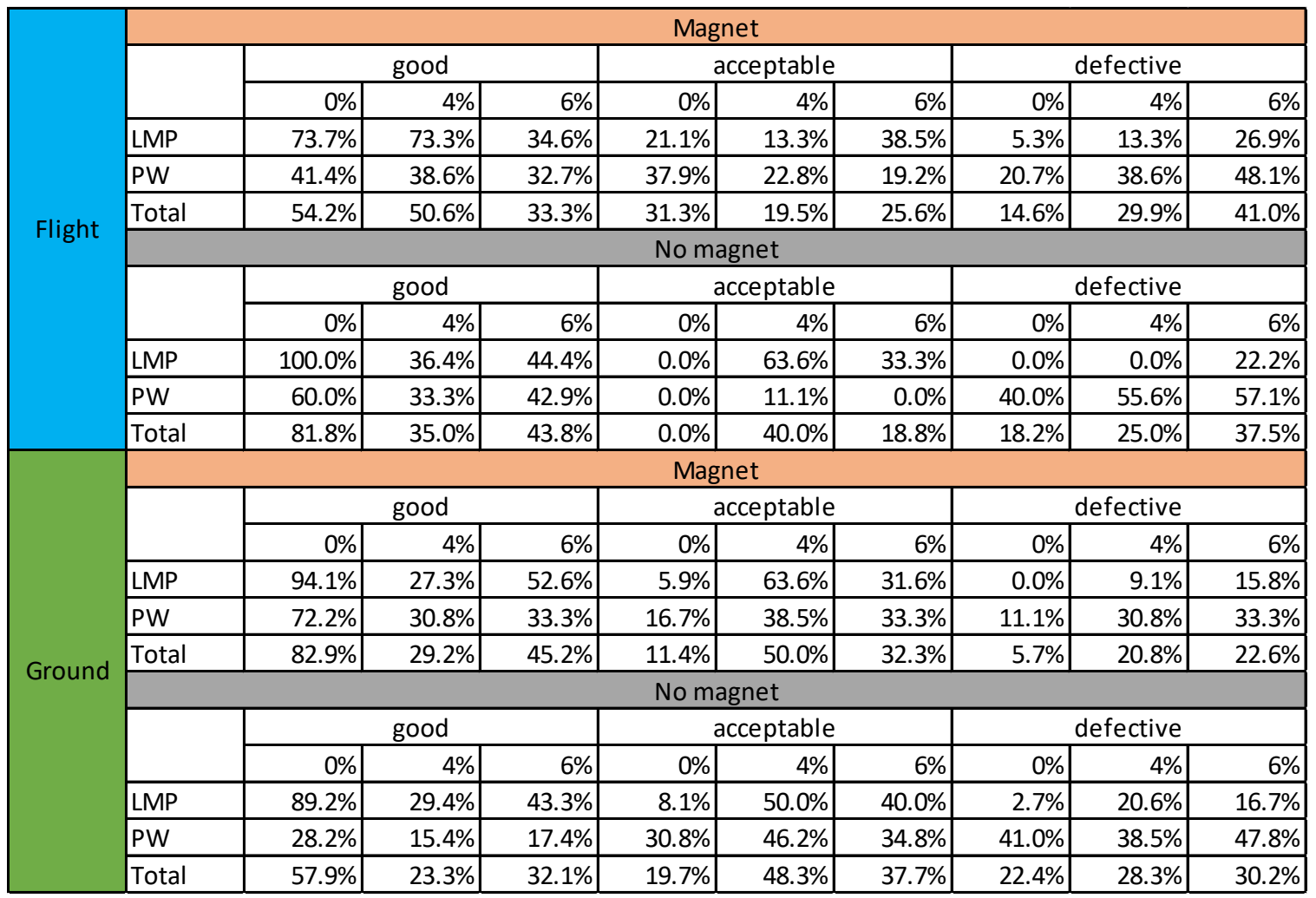

\subsection{Samples from reflow ovens and induction heating}

All the data presented above is the result of analysis of hand soldered joints.

This is the only system that will be considered for comparing data from microgravity 
and standard gravity conditions, via inspections of exterior joint quality and interior porosity. Since the induction heating system was not flown in microgravity and there is some question of whether the samples melted in the reflow ovens were sufficiently solidified during the microgravity period of the flight, these data sets will be excluded. Sufficient solidification of the joints was defined as complete solidification of the exterior of the joints, so that any gases that failed to escape during the microgravity period would remain in the solder samples permanently. Based on videos observing the experiment in flight, there is doubt that this was achieved during the intended period. Therefore, this data was excluded from the previous results. However, the samples created by the induction system and reflow ovens were processed and used in EDS analysis.

The samples created by the induction heating system had a unique feature that may give some insight on how voids form in solder joints. Melting solder samples via the induction system, described in section 3.2.5, resulted in several samples that had extremely large bubbles, in some cases greater than a millimeter in diameter. This is thought to be caused by the rapid heating and re-cooling of the samples. By limiting the time that the solder was molten, all gases contained within or created during the melting/heating process would have limited time to flow out of the solder, so the buoyant forces on any gas bubbles would be driven upward through the solder toward the top of the joint. The top of the joint was also the portion that cooled and solidified earliest in these samples due to being the farthest from the heating source and exposed to free air. These factors contributed to the 
formation of a shell of solid solder on the top surface of the joint which trapped gases inside the joint while the interior remained molten to allow bubbles to rise upward until encountering this solid shell. The size of the bubbles and consistency of their location just below the top surface of the joints supports this hypothesis.

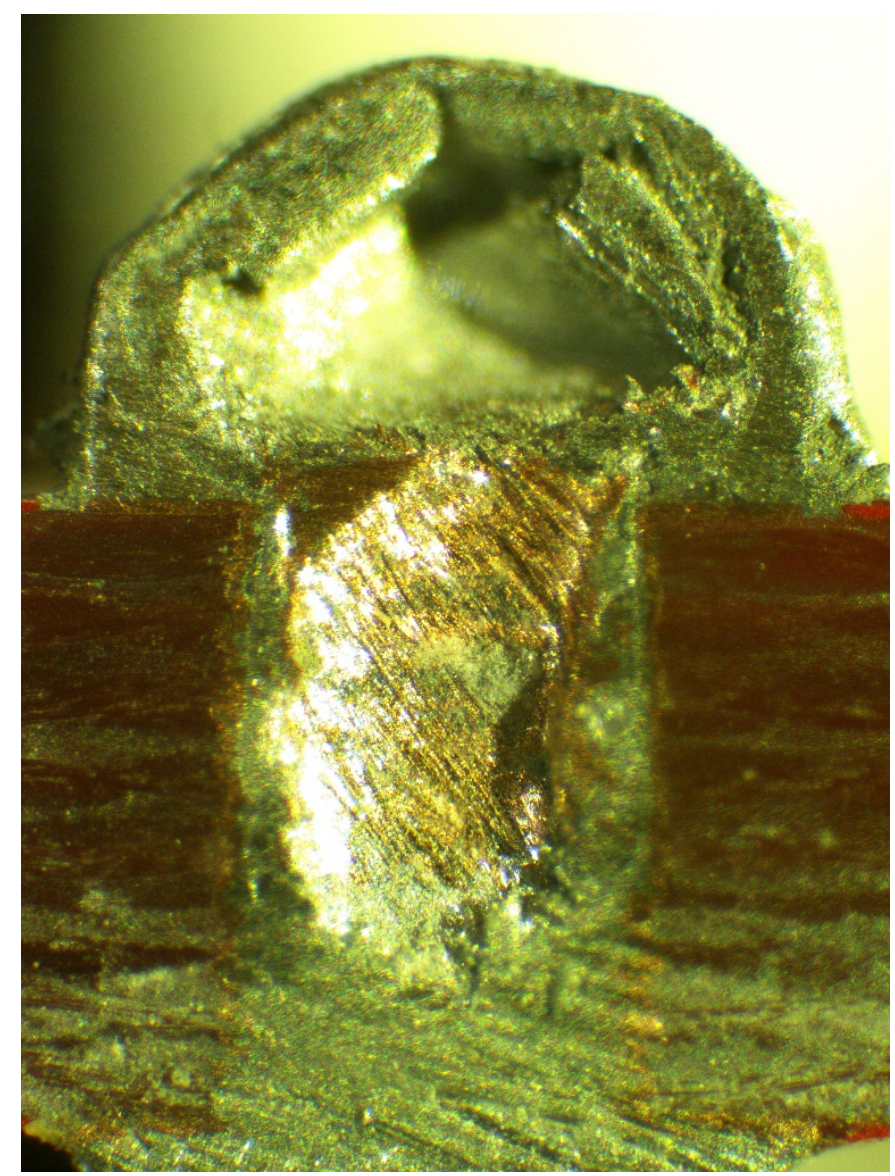

Figure 27: A joint from the induction heating system featuring a large void just beneath the top surface

\subsection{Interior Joint Porosity}

The second stage of analysis, that concerning the interior porosity of the joint quantitatively, only considers the joints created by the hand soldering stations. 
The interior joint porosity uses the same joints as the sample groups as the visual inspection evaluation, but a smaller group thereof, due to some samples being unusable for various reasons. These include: the joints created during the first five parabolas of the flight which were not formed in true microgravity, samples that were damaged during the processing, or microscope images were not of good enough quality to be converted to tricolor images. In total, 280 of the 599 hand soldered joints created moved on to interior porosity analysis (79 ground and 201 flight).

As with the visual inspection data of the samples, the effects of a few factors will each be considered separately. Again, the analysis begins with the effect of soldering iron temperature. Table 10 and Table 11 contain the data for the average interior voidage of joints separated by the iron temperature that was used to solder the joints. Table 10 leaves the data expanded to view the effect that temperature had in combination with the other solder and environmental factors being considered. Table 11 simplifies this to an overall view of how the temperature effected the two types of solder ignoring the influence of other factors. These tables indicated that contrary to the results of the visual inspection, there is no distinguishable correlation between the soldering iron temperature used and the quality of the joints, as the average interior voidage follows no pattern when the temperature is adjusted. However, it is again noted that the LMP solder paste performed better than the PW. 
Table 10: Effect of soldering iron temperature on the voidage of joints within each category

\begin{tabular}{|c|c|c|c|c|c|c|c|c|c|}
\hline \multicolumn{10}{|c|}{ Flight } \\
\hline \multicolumn{5}{|c|}{ No magnet } & \multicolumn{5}{|c|}{ Magnet } \\
\hline & $\mathrm{FE}$ & low & med & high & & $\mathrm{FE}$ & low & med & high \\
\hline \multirow{3}{*}{ LMP } & 0 & $x$ & 8.046 & 5.418 & LMP & 0 & $x$ & 4.602 & 4.766 \\
\hline & 4 & $x$ & 5.048 & 2.014 & & 4 & $x$ & 4.453 & 6.952 \\
\hline & 6 & $x$ & 13.199 & 1.573 & & 6 & $x$ & 2.066 & 2.066 \\
\hline \multirow{3}{*}{ PW } & 0 & 2.032 & 5.479 & $x$ & PW & 0 & 8.113 & 7.548 & 9.205 \\
\hline & 4 & 1.705 & 14.004 & 2.360 & & 4 & 5.593 & 6.182 & 9.398 \\
\hline & 6 & $x$ & 1.292 & 2.543 & & 6 & 2.920 & 7.961 & 6.300 \\
\hline \multicolumn{10}{|c|}{ Ground } \\
\hline \multicolumn{5}{|c|}{ No magnet } & \multicolumn{5}{|c|}{ Magnet } \\
\hline & $\mathrm{FE}$ & low & med & high & & $\mathrm{FE}$ & low & med & high \\
\hline \multirow{3}{*}{ LMP } & 0 & 1.230 & 1.093 & 0.907 & LMP & 0 & 1.114 & 4.152 & 10.141 \\
\hline & 4 & 1.190 & 0.803 & $\mathrm{X}$ & & 4 & 1.652 & 1.394 & $x$ \\
\hline & 6 & 1.036 & 1.787 & $\mathrm{x}$ & & 6 & 1.614 & 1.989 & 1.460 \\
\hline \multirow{3}{*}{ PW } & 0 & $x$ & 4.431 & 2.388 & PW & 0 & $x$ & 9.277 & $x$ \\
\hline & 4 & 2.340 & 5.253 & $\mathrm{X}$ & & 4 & 4.338 & 1.466 & 5.121 \\
\hline & 6 & 3.840 & 6.752 & 11.650 & & 6 & 6.887 & 2.282 & $x$ \\
\hline
\end{tabular}

Table 11: Average percent voidage based on temperature and solder only

\begin{tabular}{|l|c|c|c|}
\hline \multirow{2}{*}{} & \multicolumn{3}{|c|}{ Void Percent } \\
\cline { 2 - 4 } & Low Temp & Med Temp & High Temp \\
\hline LMP & 1.32 & 5.08 & 4.11 \\
\hline PW & 5.30 & 7.03 & 6.94 \\
\hline
\end{tabular}

The next factor, the solderers themselves, yields the results presented in Table 12. The highest and lowest average porosity values seen were both created by Solderer 2, as denoted in 4.1 Vision Inspection (First Round Qualitative Analysis), with the flight samples having significantly more voidage than the ground samples. This decrease in interior quality is opposite to the change seen between this individual's two sample boxes seen during the exterior quality evaluation. A similar trend, although to a lesser degree, is seen in Solderer 1's voidage data, again the porosity of the joints rose when in microgravity. Table 12 also indicates some other trends in the voidage, the ground samples having lower porosity than 
the flight samples, and iron additives and magnets not having a clearly discernable effect on the interior quality of the joints.

Table 12: Average joint porosities in each sample box

\begin{tabular}{|c|c|c|c|c|c|c|c|c|}
\hline & \multirow[b]{2}{*}{$\mathrm{Fe}$} & \multicolumn{4}{|c|}{ Flight } & \multicolumn{3}{|c|}{ Ground } \\
\hline & & Box 1 & Box 2 & Box 3 & Box 4 & Box 1 & Box 2 & Box 3 \\
\hline \multirow{3}{*}{ LMP } & 0 & 6.732 & 7.562 & 3.801 & 2.988 & 1.042 & 4.644 & $x$ \\
\hline & 4 & 3.558 & 10.559 & 2.037 & 2.442 & 0.748 & 1.523 & 2.184 \\
\hline & 6 & 8.032 & 6.047 & 1.529 & 4.055 & 1.036 & 1.601 & 1.787 \\
\hline \multirow{3}{*}{ PW } & 0 & 3.755 & 11.668 & 4.146 & 7.815 & 3.388 & 9.277 & 2.953 \\
\hline & 4 & 5.914 & 8.467 & 3.469 & 8.397 & 2.547 & 3.961 & 1.141 \\
\hline & 6 & 2.230 & 7.936 & 2.387 & 7.994 & 8.504 & 5.736 & 0.788 \\
\hline \multirow[t]{2}{*}{ Total } & all & 5.462 & 8.701 & 2.993 & 5.763 & 3.386 & 3.761 & 1.842 \\
\hline & & Solderer 1 & Solderer 2 & & & Solderer 1 & & Solderer 2 \\
\hline
\end{tabular}

The observation of higher porosity in flight samples than ground samples is consistent with the results presented by Watson, et al [8], that soldering in a microgravity environment results in more and/or larger voids within the solder joints. Nearly all the sample boxes, regardless of the presence of magnets, followed this trend and performed better in ground testing than in flight testing. When grouping all of the samples from each set of conditions together, rather than dividing them by the sample box that produced them, as in Table 13, there is also evidence of an overall better quality in the ground samples, but there are some categories of joints that either don't follow the trend (PW6 without a magnet and PW0 with a magnet both performed better in flight) or are too similar to make a worthwhile judgement (example: LMP0 with a magnet $4.693 \%$ in flight and $4.644 \%$ on ground). Looking at the standard deviation of the samples, as shown in Table 14 , shows that the variation in joint porosity is relatively large. Because of this, the 
trends in the averages are not definitive, but as this is the data produced by the experiment, these are the trends conclusions that can be made from this experiment.

Table 13: Average joint porosity for each category of solder and environmental conditions

\begin{tabular}{|c|c|c|c|c|c|}
\hline \multicolumn{6}{|c|}{ Average Porosities of Joints } \\
\hline & & \multicolumn{2}{|c|}{ Flight } & \multicolumn{2}{|c|}{ Ground } \\
\hline & & No magnet & Magnet & No magnet & Magnet \\
\hline & $\mathrm{FE}$ & porosity & porosity & porosity & porosity \\
\hline \multirow{3}{*}{ LMP } & 0 & 6.7 & 4.7 & 1.0 & 4.6 \\
\hline & 4 & 3.6 & 5.8 & 1.0 & 1.5 \\
\hline & 6 & 8.0 & 4.8 & 1.4 & 1.6 \\
\hline \multirow{3}{*}{ PW } & 0 & 3.8 & 8.4 & 3.3 & 9.3 \\
\hline & 4 & 5.9 & 7.4 & 3.5 & 4.0 \\
\hline & 6 & 2.2 & 6.5 & 7.7 & 5.7 \\
\hline
\end{tabular}

Table 14: Standard deviations of averages presented in Table 13

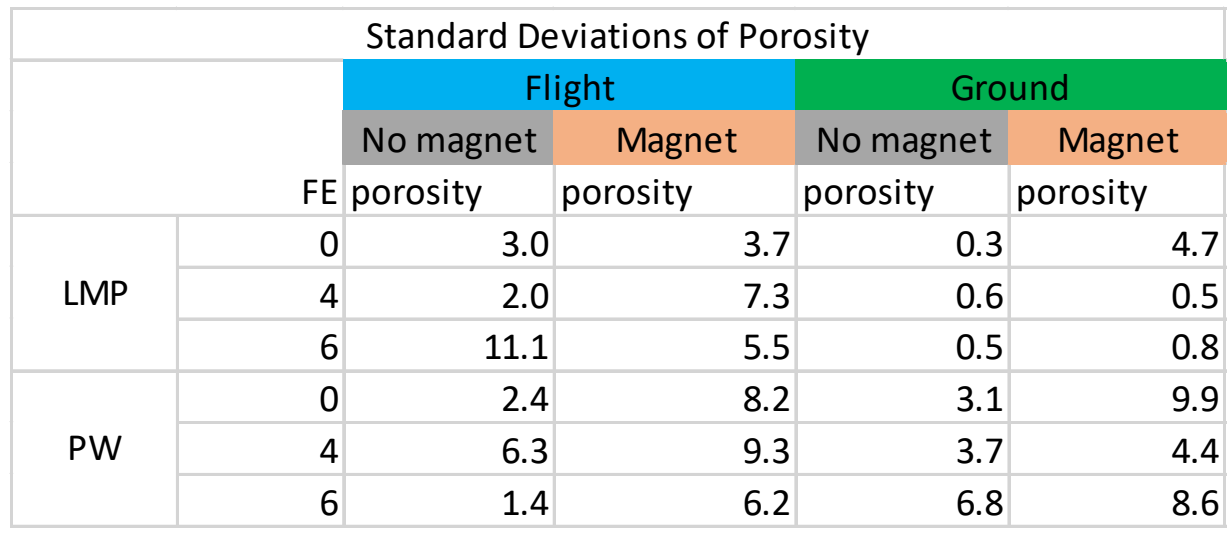

Since the trends in the analysis between exterior quality and interior quality don't correspond, a table was created to compare the two analyses. Table 15 explores whether the joints' interior porosity would be related to their evaluated exterior quality. Based on this table there appears to be no correlation between the 
evaluated exterior and interior quality of the joints. This indicates that for overall quality of the joints, the interior quality of the joints is independent of the exterior. Therefore, the intended improvement of the interior, which was the basis of the experimentation with iron additives and magnets, cannot be evaluated by the easily judged surface inspection.

Table 15: Interior voidage percent compared to visual joint quality

\begin{tabular}{|c|c|c|c|c|c|}
\hline \multicolumn{6}{|c|}{ Good } \\
\hline & & \multicolumn{2}{|c|}{ Flight } & \multicolumn{2}{|c|}{ Ground } \\
\hline & & No magnet & Magnet & No magnet & Magnet \\
\hline & $\mathrm{Fe}$ & porosity & porosity & porosity & porosity \\
\hline \multirow{3}{*}{ LMP } & 0 & 6.732 & 5.318 & 1.042 & 5.457 \\
\hline & 4 & 5.431 & 6.877 & 0.859 & $x$ \\
\hline & 6 & 9.283 & 6.931 & 1.218 & 5.584 \\
\hline \multirow{3}{*}{ PW } & 0 & 5.479 & 8.395 & 3.846 & 9.277 \\
\hline & 4 & 2.495 & 5.469 & 3.979 & 5.584 \\
\hline & 6 & 2.791 & 5.566 & 8.080 & 1.944 \\
\hline \multicolumn{6}{|c|}{ Acceptable } \\
\hline & & \multicolumn{2}{|c|}{ Flight } & \multicolumn{2}{|c|}{ Ground } \\
\hline & & No magnet & Magnet & No magnet & Magnet \\
\hline & $\mathrm{Fe}$ & porosity & porosity & porosity & porosity \\
\hline \multirow{3}{*}{ LMP } & 0 & $x$ & 3.265 & $x$ & 0.577 \\
\hline & 4 & 3.023 & 3.849 & 1.079 & 1.714 \\
\hline & 6 & 10.601 & 3.645 & 1.994 & 4.398 \\
\hline \multirow{3}{*}{ PW } & 0 & $x$ & 7.406 & 1.904 & $x$ \\
\hline & 4 & 13.271 & 11.492 & 3.941 & 4.398 \\
\hline & 6 & $x$ & 5.391 & 7.004 & 2.282 \\
\hline \multicolumn{6}{|c|}{ Defective } \\
\hline & \multicolumn{3}{|c|}{ Flight } & \multicolumn{2}{|c|}{ Ground } \\
\hline & & No magnet & Magnet & No magnet & Magnet \\
\hline & $\mathrm{Fe}$ & porosity & porosity & porosity & porosity \\
\hline \multirow{3}{*}{ LMP } & 0 & $x$ & 2.277 & $x$ & $x$ \\
\hline & 4 & $x$ & 1.568 & $x$ & 0.951 \\
\hline & 6 & 1.676 & 5.751 & 1.217 & 2.295 \\
\hline \multirow{3}{*}{ PW } & 0 & 2.032 & 10.546 & $X$ & $x$ \\
\hline & 4 & 4.930 & 7.024 & 4.271 & 2.295 \\
\hline & 6 & 1.670 & 8.270 & 8.030 & 9.358 \\
\hline
\end{tabular}


These results suggest that the only condition that had a consistent and significant effect on the interior joint porosity is the gravitational level. The combination of iron and magnets did not have enough of an effect on the solder to replicate the effects of gravitational force on the molten solder. Neither the iron additives, the soldering iron temperature, or the magnets had much effect on their own.

\subsection{Energy Dispersive X-ray Spectroscopy}

Several samples were pulled from the main sample group to undergo EDS analysis to determine the source of voids in the solder joints. The hypothesis in this testing was that there may be traces of the gases that form the bubble left behind on the surface of the cavity. Based on this hypothesis, since the fluxes were mostly organic compounds, the amount of carbon, hydrogen, and oxygen present in the cavity was observed and compared to the cross-sectional surface of the solder. More carbon, hydrogen, and oxygen in the cavities, compared to the solder surface, would support the idea that the voids are created by vaporized flux as the organic fluxes in the solders are comprised primarily of these elements. Finding higher levels of hydrogen and oxygen with no significant increase in carbon would suggest that these bubbles are made up of water vapor either released from the circuit boards or taken in from the environment. 
Figure 28 presents an example of the output of EDS, when observing an area, the machine outputs an image of the area being observed and a mapping of the atomic species detected in that area with each colored dot on the map corresponding to the detection of a certain type of atom in that location. This map also provides images that contain only one type of atom, like shown in Figure 29, where all but the carbon is removed from the map.
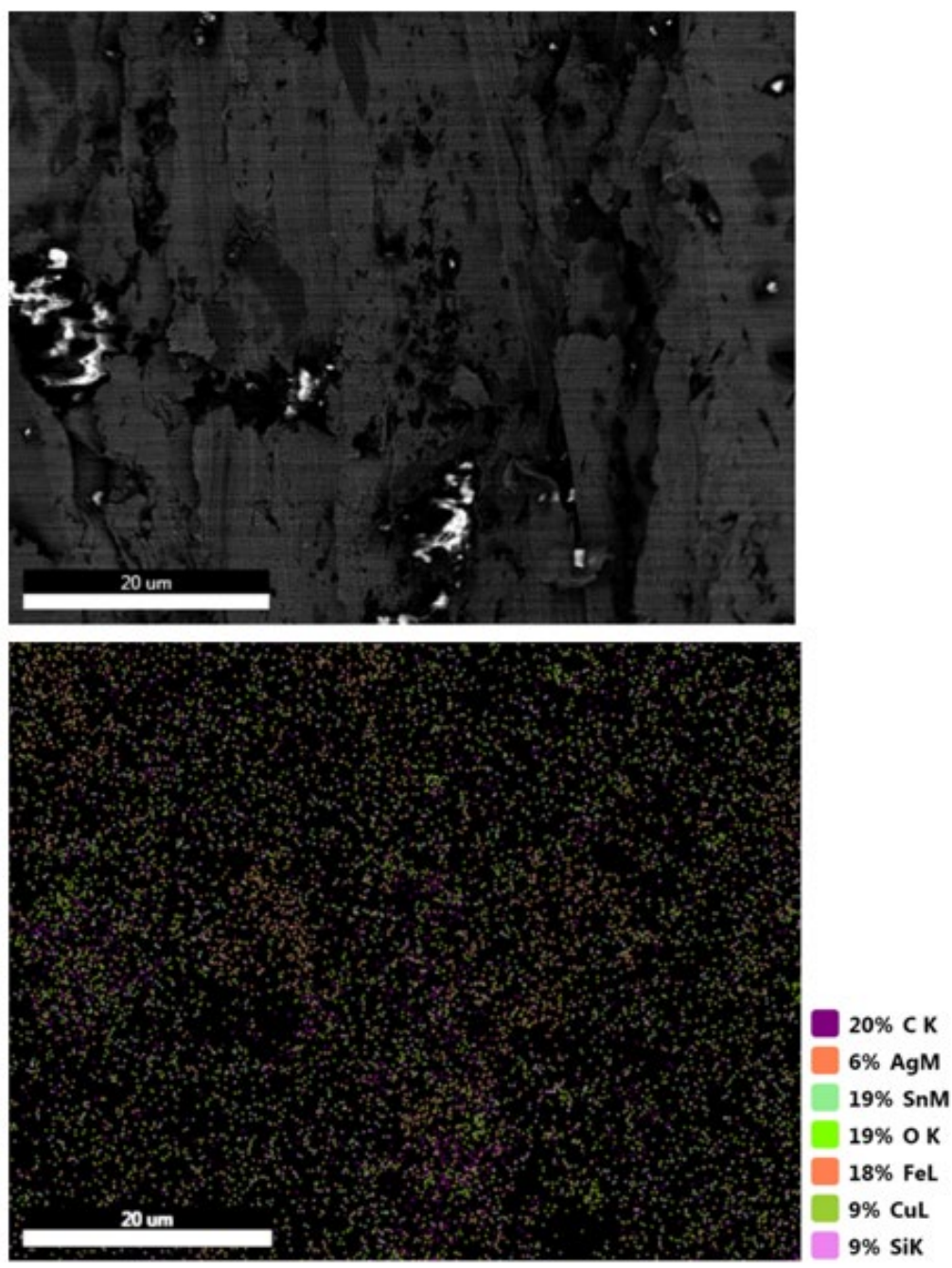

Figure 28: An example of the output from EDS, an image of the area being observed and the mapping of atomic species detection 


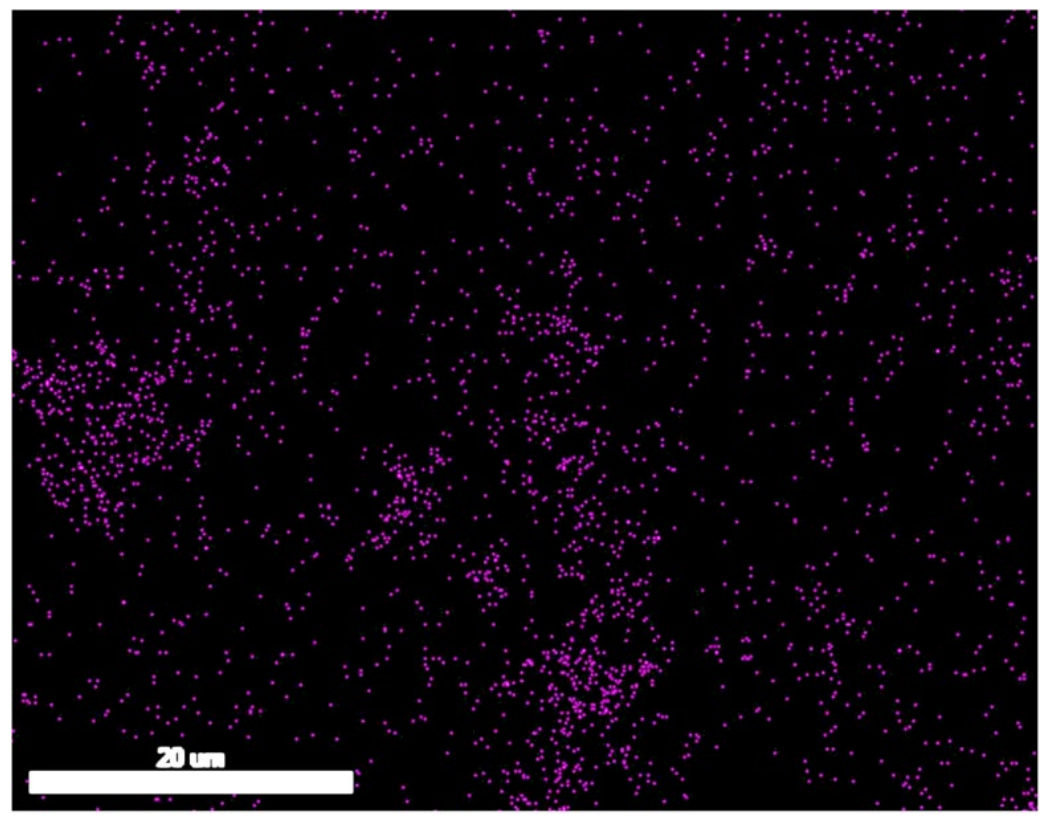

Figure 29: The area mapped in Figure 28, but showing only the presence of carbon

Early observations showed carbon to be the majority element on the surface of the cavities, which led to a belief that the bubbles were caused by flux vapor caught in the solder joint at the time of solidification. However, this was called into question when later observation of a sample with deep scratches on the surface from inadequate polishing showed higher amounts of carbon in the bottom of the scratches compared to the flatter surface areas. Figure 31 displays this tendency for scratches to be mapped high in carbon. Due to that observation it was concluded that the carbon observed was not necessarily due to vapor residue on the interior of the voids, but rather more likely came from contamination during the polishing process. This leaves the source of the voids unproven and indicates that the cross-sectioning process used to view the interior of these samples may prevent analysis of the makeup of the interior of the voids from being determined. 


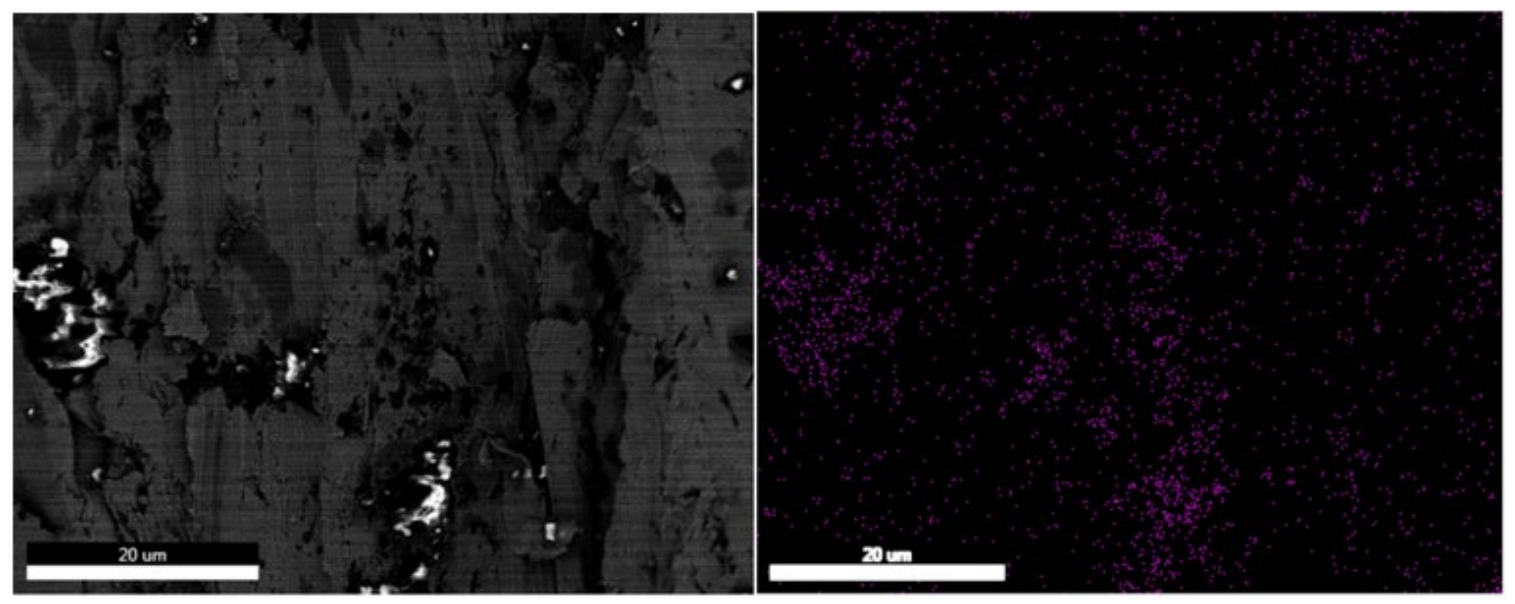

Figure 30: EDS carbon map from the interior of a large void, 20\% Carbon

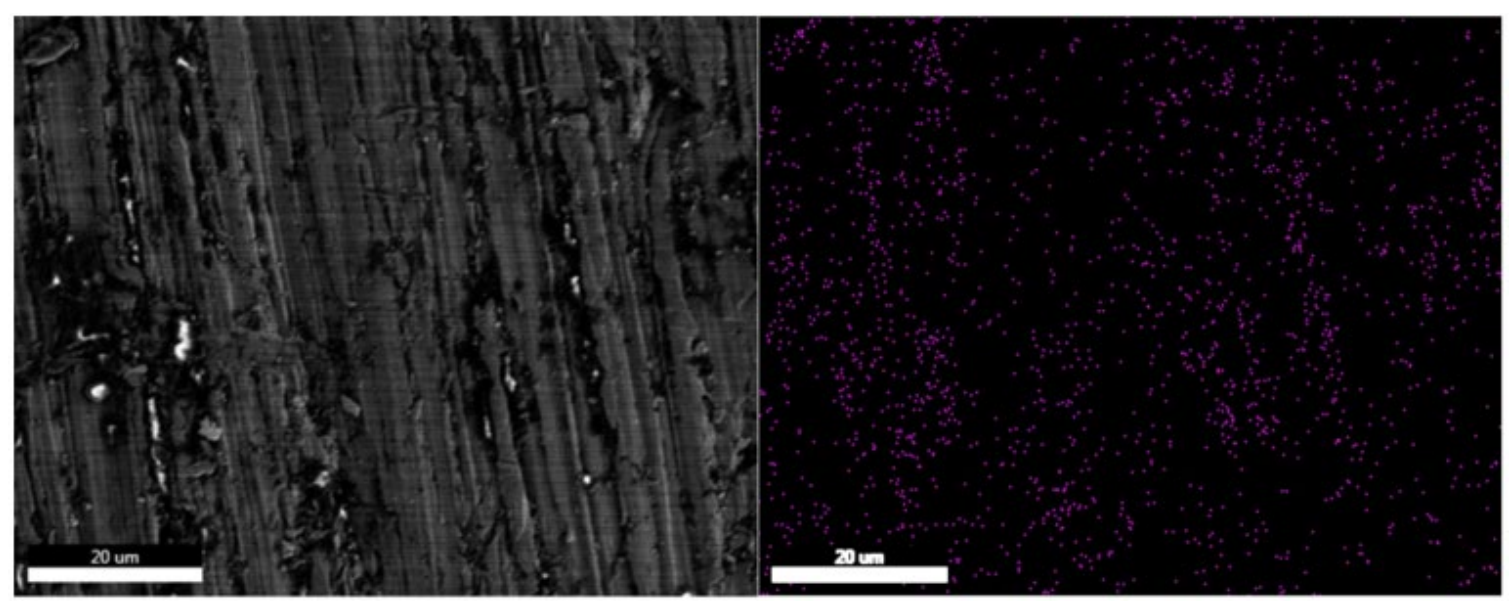

Figure 31: EDS carbon map from the polished surface of the same joint that Figure 30 was taken from, $18 \%$ carbon

During the EDS analysis, the areas around voids were examined looking for concentrations of iron particles to determine if the iron dispersoid created a blockage to the flow of the bubbles. None of the EDS images showed an increase in the amount of iron near voids, so the iron was not responsible for stopping the flow of the gases, but may have slowed the flow. This cannot be fully determined from EDS analysis. 


\section{Conclusions}

This experiment began with three goals: verify or refute previous results that solder joints made in microgravity have higher porosity than those made on Earth, investigate the source of the vapor bubbles that lead to joint porosity, and study the effects of a combination of iron additives and magnetic fields to determine if this was a viable way to reduce joint porosity. The results obtained suggest concurrence with previous researchers' results that joints created in microgravity generally have more voidage than those created in normal gravitational conditions. The investigation into the cause of vapor bubbles in solder was inconclusive but did provide some insight into the problem. And the attempt to use iron microparticles and magnetic fields to affect the flow of bubbles and therefore porosity seems at this point to have been inconclusive.

The data suggesting that the joints were of poorer quality in microgravity was an expected result since this result was seen by previous researchers. Seeing similar results here validates not only that the previous results were accurate, but also that there is some problem with soldering in microgravity that is worth investigating. If not for the difference in porosity between the two gravitation environments, the testing of the effects of the iron dispersoids would have been of little benefit. Although this experiment did not directly explore the reasons that the voidage is higher in microgravity, there are some hypotheses for this. 
The gravity force on the solder would provide a buoyant force that gives the bubbles in the molten solder a direction to flow. The large cavities in the top of the joint created by the induction heating system indicate that there is flow of the gases toward the upper surface of the joint, but without gravity there is no buoyant force to drive the flow of the bubbles, so they are more likely to remain sedentary and still be present in the joint at the time of solidification.

In trying to determine the composition of the bubbles, none of the suspected sources were directly determined to be the primary cause of voidage and the contents of the bubbles remains unknown. However, through some of the observations in this experiment, there are clues to the sources. Flux is likely to have been the main contributor to the bubbles in the solder, since most of the flux burns off and escapes as smoke during soldering. However, the fact that the flux is evenly distributed throughout the solder paste means that some of the smoke will form within the molten solder and need to flow out to prevent remaining in the joint as a void once the solder solidifies.

The EDS observation of the interior of the joints failed to prove that there were deposits of flux residue on the interior of the voids because the method for cross-sectioning the joints left contaminates from the polishing papers on all nonplanar surface of the joint (voids, scratches, divots) that couldn't be adequately distinguished from what was expected of the flux vapor residue. It is likely that there were more sources contributing to the bubbles than just the flux vapors. 
The fact that this experiment used solder paste rather than a solid wire solder may have played a part. Several steps in the process of preparing the pastes and applying them to the boards allow for opportunities for air to be mixed in or trapped in the paste. During the mixing to add the iron microparticles, the mixture was stirred to distribute the particles throughout the solder. The paste was then transferred to syringes that were used to apply the paste to boards, and even when the paste was being applied there was some chance of air being trapped between layers of the paste or between the paste and the board. At any stage when the paste was being manipulated or moved prior to soldering there was opportunity for air to be trapped by or mixed in with the solder paste.

Also, water vapor, which both Watson et al [8] and Easton et al. [9] pointed to as a potential source of bubbles would likely have contributed to the porosity. This experiment made no attempt to measure the effect of the water vapor coming from within the boards themselves, but Watson et al [8] results did indicate that baking the boards before using them reduced the water in the boards resulting in less water vapor from outgassing of the boards made its way into the joints. Since this experiment took no measures to decrease the water within the boards, some contribution to the bubbles from the water vapor is likely.

Each of the hypothesized sources of the bubbles in the joints from the previous paragraph could be tested to determine their effect on the porosity of the 
joint to determine how much of a role in the overall development of the bubbles each plays. The effect of flux vapor could be tested by repeating the test using fluxless solders. Traditionally, fluxless solders are not used because the flux cleans the surface and allows for better adherence of the solder, but, for the sake of determining the effect of flux on voidage, fluxless solder could be used to create joints to create solder samples for determination of interior porosity.

The second source stated, air in the paste, could be tested by using wire solder, which is the standard for this type of soldering and was not used in the present only because using paste more easily allowed for inclusion of the iron additives. With wire solder, there would be no air in the solder that could potentially form bubbles in the joint. Water vapor moving from the board to the joint is an effect that has previous been explored. Watson, et al. [8] baked boards before using them to reduce the amount of water present in the boards, this in turn lead to a reduction in the porosity of the joints.

The lack of consistent results from the inclusion of iron additives in the solder paste does not necessarily indicate that the idea will not work, but rather that in this experiment was of little to no benefit. With the samples that contained iron, there were some added negative effects that the interaction between the iron particles and magnets had to overcome to potentially see a positive effect from the dispersoid. The solid dispersoids would create obstacles to the flow of gases as 
they move through the liquid solder, this would slow the movement of the gases and increase the likelihood that they remain present at the time of solidification.

Based on the data presented in 4.1 Vision Inspection (First Round Qualitative Analysis), the joints with higher iron content were more likely to have defects. As stated in that section, this is likely due to the increase in viscosity brought on by the solid particles in the solder. To overcome both problems, the time that the solder is molten could be increased, allowing the bubbles more time to move through the solder. This would oppose the negative effect on the flow from the increased viscosity and give them more time to navigate around the solids that obstruct their movement. The manufacturer of the solder pastes used in the experiment recommends that the solder be molten for a period of approximately 40 seconds. This amount of time is not possible during a microgravity flight because the plane is only in microgravity conditions for $15-20$ seconds at a time, but using a molten period of 15 seconds may yield lower voidage results than the 5-7 second period the solderers on this flight used.

The effect of the iron could also be increased by using a stronger magnetic field; in a stronger magnetic field the iron particles would have a more powerful force pulling them down into the hole in the board. The iron moving toward the magnet drags solder along with it and provides a source of motion toward the bottom of the hole. This force was present during the current experiment, but since no trend of change in porosity with iron content was observed, increasing the 
magnetic force on the joint by either using stronger magnets or moving the boards closer to the surface of the boards would be worth exploring.

In summary, the results of this experiment supported previous researchers results that solder joint porosity increased for joints created in microgravity, the source of the bubbles that form voids was not determined, but the hypothesized sources are all thought to have some contribution to the total porosity. And the use of iron microparticles in conjunction with a magnetic field provided no conclusive result for either positive or negative effects on the overall joint quality. 


\section{Future Work}

MRT 14 has continued this research with a second, similar flight conducted in November of 2018. This second flight served as a continuation of the previous flight, gathering more samples in microgravity, and an opportunity to explore new solders and methods of soldering and processing samples.

Improvements to the solder that were tested included: an effort at making solder wires that contain iron particles as an additive to replace the solder pastes used in previous testing, developing solders that do not include flux, and coating iron particles in silver before adding them into the solder.

The use of solder wires is more common in hand soldering than solder pastes. Therefore, being able to use solder wire in testing is a more accurate representation of how an individual would likely solder while making a minor repair. This would give the person soldering better control over the placement and amount of solder used when compared to the pre-prepared boards utilizing solder paste as well as allowing the solderer to better observe the flow of solder into the joint.

The use of fluxless solder would eliminate a potential source of vapor from the soldering process, as without the vaporized flux in the joint, the only remaining potential sources of gas bubbles would be the surrounding air and any water vapor released during the soldering process. By using reactive metals in place of traditional fluxes, it is hypothesized that these additives could strip any oxidized layer from the soldering surface leaving a clean metal surface for the solder to adhere to. 
Coating iron particles in silver is also being explored, as it is potentially a way to introduce iron particles that will better wet to the rest of the solder. This may allow for the addition of more particles before clumping of the iron is seen, which would in turn prevent the iron particles from being drawn out of the solder once the magnetic field is applied.

In addition to these improvements to the solder, two improvements to the experiment setup were also tested. One change to the hand soldering setup was made to increase the magnetic field, and a new method for melting and testing solder samples, specifically melting them in a test section that can be used to quantify their shear strength.

The first experiment improvement was simply increasing the strength of the magnetic field at the location of the board. This was achieved by moving the board closer to the magnets in the sample boxes. This would increase the force acting on the iron in the solder and possibly result in better flow of the molten solder. Increasing the magnetic pull on the iron will help determine if using magnetic force to replace the gravity force is beneficial to the joints.

The second experiment improvement gave a method for quantifying the strength of the joints. By melting solder between two copper plates, a stress test can be performed on the solder section. The joined copper plates can be inserted into an Arcan disc [25], so that both the shear and tensile strength can be tested 
using an available tensile testing machine (see Figure 32). A test of this nature was previously performed by Imperial College London [7] to quantify the reduction of strength of microgravity solder joints. WVU's MRT conducted a similar experiment on its most recent flight to verify these results and compare strength data for solder joints using the magnetic solder paste to those formed under nonmagnetic conditions, both in microgravity and normal gravity environments.

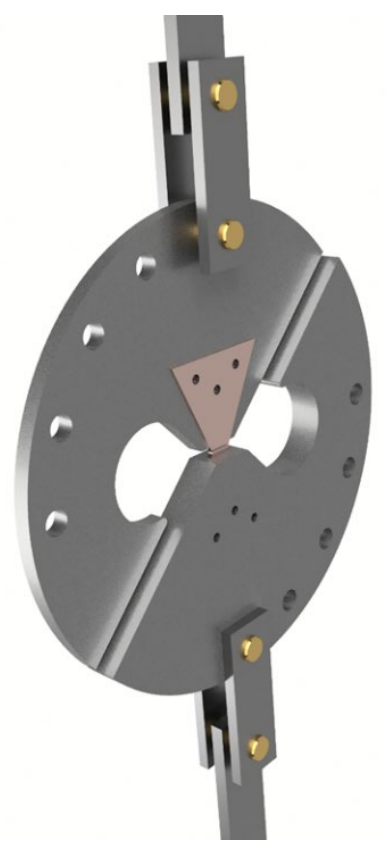

Figure 32: A model of an Arcan disk that could be used be used to test the strength of solders

Not all of these intended improvements to the solder were developed in time to be incorporated in the November 2018 flight. Forming the solder into a more usable wire form, was not accomplished, nor was the development of a fluxless solder. However, the silver coated iron particles were added to some of the solders and used during ground testing, but whether it was beneficial was inconclusive. 
Because of this it was not used in flight, so that students working on this could be diverted to other areas of the project. The result of this was that the second flight replicated the solders used in the initial flight to generate the new samples.

Although the improvements to the solder were not implemented, both proposed changes to the experiment setup were. The distance between the magnets and the sample boards was cut in half for the second flight and the system for testing the strength of the joints has been manufactured and the joints for its testing soldered both in the lab and in microgravity. However, these samples from the changes have not yet been tested, so there are not yet any results available. 


\section{References}

[1] R. D. Pettegrew, P. Struk, J. Watson and D. R. Haylet, "Experimental Methods in Reduced-Gravity Soldering Research," NASA TM 2002-211993, 2002.

[2] R. D. Pettegrew, J. Easton, P. Struk and E. Anderson, "In-Flight Manual Electronics Repair for Deep-Space Missions," in 44th AIAA Aerospace Sciences Meeting and Exhibit, Reno, NV, 2006.

[3] R. D. Pettegrew, J. E. Peter Struk, G. Gorecki and D. Truong, "Repair of Electronics for Long Duration Spaceflight," in 45th AIAA Aerospace Sciences Meeting and Exhibit, Reno,NV, 2007.

[4] R. C. Oeftering, R. P. Wade and A. Izadnegahdar, "Component-Level Electronic-Assembly Repair (CLEAR) Spacecraft Circuit Diagonostics by Analog and Complex Signature Analysis," NASA TM 2011-216952, 2011.

[5] R. C. Oeftering, M. A. Bradish, J. R. Juergens, M. J. Lewis and D. R. Vrnak, "Component-Level Electron-Assembly Repair (CLEAR) System Architecture," NASA TM 2011-216956, 2011.

[6] A. Accola, H. J. Fincannon, G. J. Williams and R. T. Meier, "Sensitivity Study of Space Station Freedom Operations Cost and Selected User Resources," in IAA Symposium on Space Systems Cost Estimation Methodogies and Applications, San Diego, CA, 1990. 
[7] B. Thomas, A. Atkinson and R. Dashwood, "Strength of Soldered Joints Formed under Microgravity Conditions," Journal of Electronic Materials, vol. 36, no. 1, pp. 1-5, 2007.

[8] J. Watson, P. Struk, R. Pettigrew and R. Downs, "Experimental Investigation of Solder Joint Defect Formation and Mitigation in Reduced-Gravity Environments," Journal of Spacecraft and Rockets, vol. 44, pp. 174-182, 2007.

[9] J. W. Easton and P. M. Struk, "Soldering in a Reduced Gravity Environment (SoRGE)," NASA/TM 2012-217119, 2012.

[10] T. Carlberg and M. Liljendahl, "Soldering Under Microgravity," in 4th European Symposium on Materials Science under Microgravity, Madrid, Spain, 1983.

[11] A. H. Habib, M. G. Ondeck, K. J. Miller, R. Swaminathan and M. E. McHenry, "Novel Solder-Magnetic Particle Composites and Their Reflow Using AC Magnetic Fields," IEEE Transactions on Magnetics, vol. 46, no. 6, pp. 21872190, 2010.

[12] H. Mavoori and S. Jin, "New, Creep-Resistant, Low Melting Point Solders with Ultrafine Oxide Dispersions," Journal of Electronic Materials, vol. 27, no. 11, 1998.

[13] H. Mavoori and S. Jin, "Significantly Enhanced Creep Resistance in LowMelting-Point Solders through Nanoscale Oxide Dispersions," Applied Physics Letters, vol. 73, no. 16, pp. 2290-2292, 1998. 
[14] J. D. Calabro, X. Huang, B. G. Lewis and A. G. Ramirez, "Magnetically Driven Three-Dimensional Manipulation and Inductive Heating of MagneticDispersion Containing Metal Alloys," in Proceedings of the National Academy of Sciences, 2010.

[15] H. S. Betrabet, O. H. Boser, R. H. Kane, S. McGee and T. Caulfield, "Dispersion Strengthened Lead-Tin Alloy Solder". US Patent 5066544, 19 November 1991.

[16] G. B. Alexander and R. A. Nadkarni, "Metal Articles Having a Plurality of Ultrafine Particles Dispersed therein". US Patent 5134039, 28 June 1992.

[17] F. Guo, "Composite Lead-Free Electronic Solders," Journal of Materials Science, vol. 18, pp. 129-145, 2007.

[18] J. H. Lee, D. J. Park, J. N. Heo, D. H. Shin and Y. S. Kim, "Reflow Characteristics of Sn-Ag Matrix In-Situ Composite Solders," Scripta Materialia, vol. 42, no. 8, pp. 827-831, 2000.

[19] M. McCormack, S. Jin and G. W. Kammlott, "Enhanced Solder Alloy Performance by Magnetic Dispersions," IEEE Transactions on Components, Packaging, and Manufacturing Technology, vol. 17, no. 3, pp. 452-457, 1994.

[20] S. Jin and M. McCormack, "Article Comprising Solder with Improved Mechanical Properties". US Patent 5,346,775, 1994.

[21] S. Itschner, Principal of Soldering, Morgantown, West Virginia: Lecture, 2013. 
[22] K. Phillips and J. M. Kuhlman, "Development of the WVU Small Microgravity Research Facility (SMiRF)," in 53rd AIAA Aerospace Sciences Meeting, Kissimmee, Florida, 2015.

[23] J. M. Kuhlman, B. Dietzius, G. Eberhart, C. Gary, S. Kosko, J. Milam and N. Ohi, "Development of a Microgravity Research Team Project Course," Journal of Thermophysics and Heat Transfer, vol. 30, no. 3, p. 622, 2016.

[24] IPC Electronics, Through Hole Solder Joint Evaluation; Training and Reference Guide, Bannockburn Illinois, 2014.

[25] T. H. McGaffigan, "Method, System, and Composition for Soldering by Induction Heating". US Patent 5093545, 3 March 1992.

[26] NASA Technology Roadmaps, "TA 3: Space Power and Energy Storage," NASA, 2015.

[27] NASA Technology Roadmaps, "TA 7: Human Exploration Destination Systems," NASA, 2015.

[28] M. Arcan and Z. H. A. Voloshin, "A Method to Produce Uniform Plane-Stress States with Applications to Fiber-Reinforced Materials," Experimental Mechanics, vol. 18, pp. 141-146, 1978.

[29] R. N. Grugel and L. N. Brush, "Solidification Dynamics of Spherical Drops in a Free Fall Environment," Microgravity Science and Technology, vol. 19, no. 1, pp. 32-44, 2007.

[30] R. N. Grugel, P. Luz, G. Smith, R. Spivey, L. Jeter, D. Gillies, F. Hua and A. V. Anilkumar., "Materials research conducted aboard the International Space 
Station: Facilities overview, operational procedures, and experimental outcomes," Acta Astronautica, vol. 62, pp. 491-498, 2008.

[31] S. Y. Hwang, J. W. Lee and Z. H. Lee, "Microstructure of a Lead-Free Composite Solder Produced by an In-Situ Process," Journal of Electronic Materials, vol. 31, no. 11, pp. 1304-1308, 2002.

[32] NASA Technology Roadmaps, "TA 5: Communications, Navigation, and Orbital Debris Tracking and Characterization Systems," NASA, 2015. 


\section{Appendix A: Soldering Pastes Used}

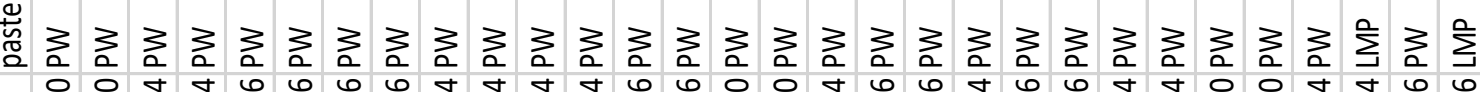

ષ

$\stackrel{2}{\stackrel{0}{2}}$

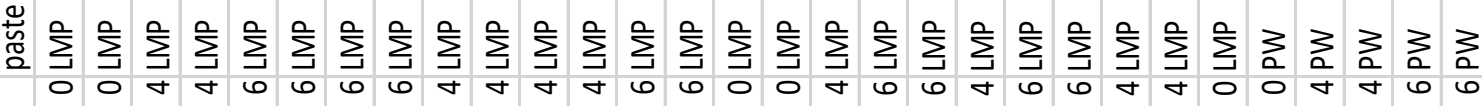
$\frac{\frac{0}{4}}{\dddot{0}}$

ㄴ.广

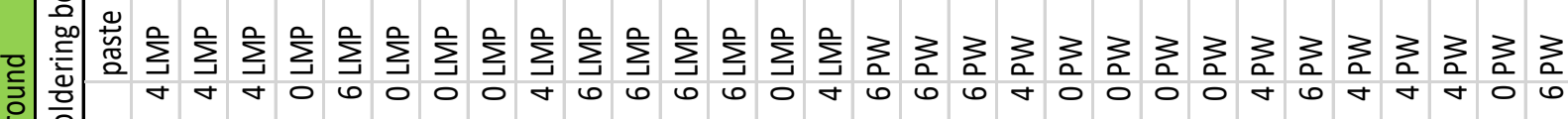

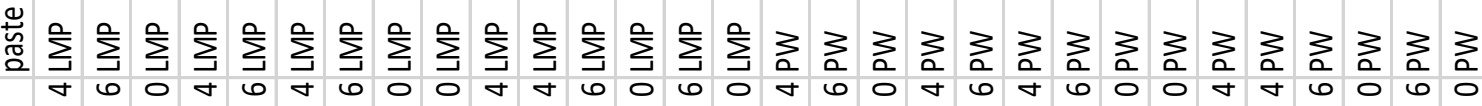
๗

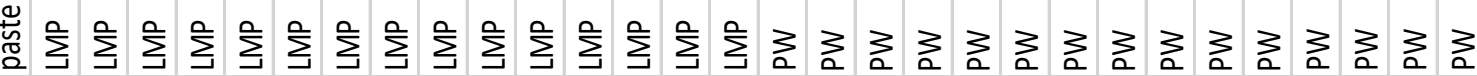

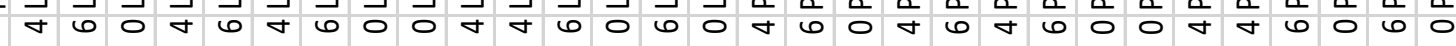
닌

$\stackrel{c}{\Phi}$

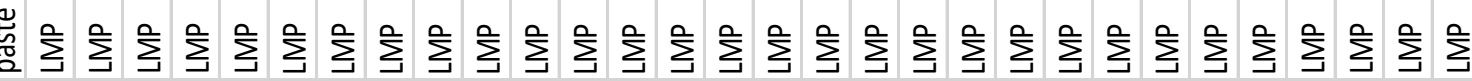
$\frac{0}{\frac{0}{4}}$

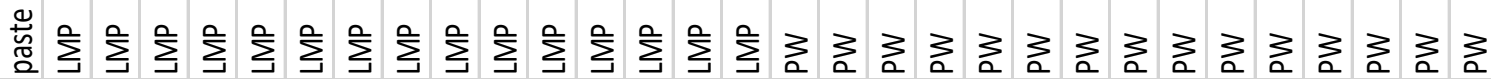

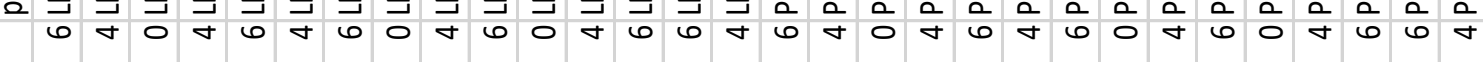
돈

¿

궁 $\stackrel{\frac{\pi}{2}}{2}$ 


\section{Appendix B: Parabolic Aircraft Gravity Data}

\begin{tabular}{|c|c|c|c|}
\hline \multicolumn{5}{|c|}{ G-Level Measurements in Flight } \\
\hline \multicolumn{3}{|c|}{} & \multicolumn{3}{|c|}{ G-level } \\
\hline Parabola \# & $10 \%$ G & $5 \%$ G & 3\% G \\
\hline 6 & -0.02017 & -0.01619 & -0.01554375 \\
\hline 7 & -0.0167 & -0.00702 & -0.005520792 \\
\hline 8 & -0.00483 & -0.00216 & -0.001405724 \\
\hline 9 & -0.01223 & -0.00395 & -0.002866055 \\
\hline 10 & -0.00258 & 0.000789 & 0.001823529 \\
\hline 11 & 0.001613 & 0.004728 & 0.005882456 \\
\hline 12 & 0.00128 & 0.0034 & 0.004090592 \\
\hline 13 & -0.00372 & -0.00169 & -0.000952899 \\
\hline 14 & -0.0092 & -0.00401 & -0.00271063 \\
\hline 15 & -0.00205 & 0.006218 & 0.00831094 \\
\hline 16 & -0.00428 & 0.005652 & 0.006926148 \\
\hline 17 & -0.00636 & 0.000497 & 0.001909266 \\
\hline 18 & -0.00525 & 0.001307 & 0.002296099 \\
\hline 19 & -0.00733 & -0.00469 & -0.00409507 \\
\hline 20 & -0.00289 & 0.000769 & 0.001708015 \\
\hline 21 & 0.000479 & 0.002794 & 0.004245315 \\
\hline 22 & -0.00464 & -0.00192 & -0.001056027 \\
\hline 23 & 0.001772 & 0.004034 & 0.004517185 \\
\hline 24 & 0.001553 & 0.004722 & 0.006073043 \\
\hline 25 & -0.00289 & -0.00064 & 0.000321027 \\
\hline 26 & -0.01217 & -0.0035 & -0.002256705 \\
\hline 27 & -0.00411 & 0.001125 & 0.002744526 \\
\hline 28 & 0.00628 & 0.008299 & 0.008894904 \\
\hline 29 & -0.01072 & -0.00861 & -0.008116585 \\
\hline 30 & 0.007238 & 0.008952 & 0.009420575 \\
\hline & & & \\
\hline
\end{tabular}

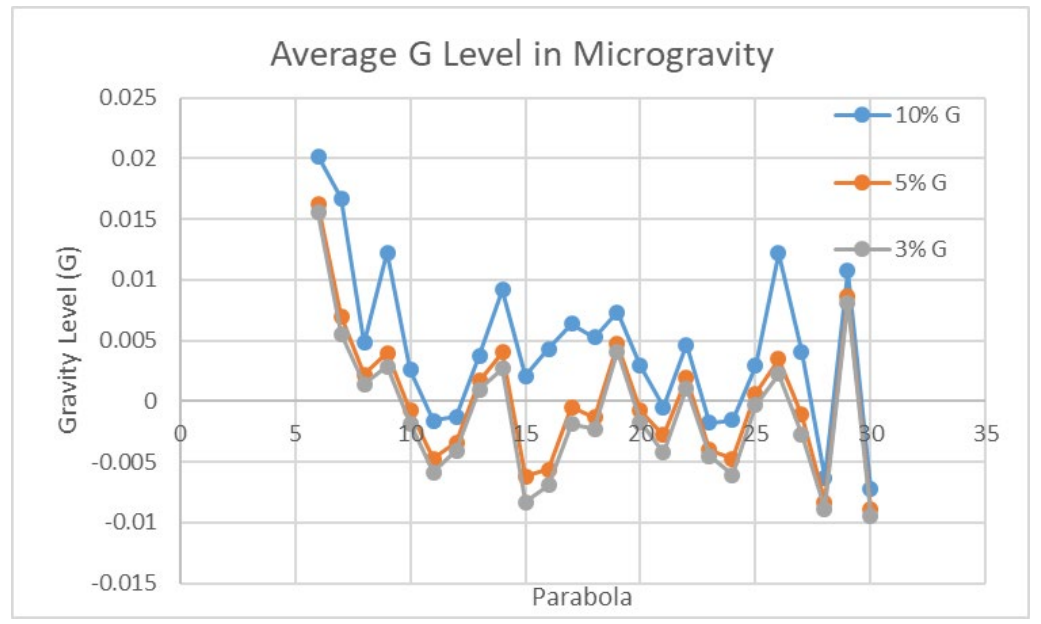




\begin{tabular}{|c|c|c|c|}
\hline \multicolumn{4}{|c|}{ In-Flight G Level RMS } \\
\hline \multirow[b]{2}{*}{ Parabola \#: } & \multicolumn{3}{|c|}{ G RMS } \\
\hline & $10 \% \mathrm{G}$ & $5 \% \mathrm{G}$ & $3 \% \mathrm{G}$ \\
\hline 6 & $2.40 \%$ & $1.90 \%$ & $1.88 \%$ \\
\hline 7 & $3.07 \%$ & $2.04 \%$ & $1.91 \%$ \\
\hline 8 & $2.77 \%$ & $2.44 \%$ & $2.39 \%$ \\
\hline 9 & $2.43 \%$ & $1.03 \%$ & $0.79 \%$ \\
\hline 10 & $2.42 \%$ & $1.85 \%$ & $1.74 \%$ \\
\hline 11 & $2.27 \%$ & $1.64 \%$ & $1.49 \%$ \\
\hline 12 & $2.13 \%$ & $1.70 \%$ & $1.62 \%$ \\
\hline 13 & $2.47 \%$ & $2.17 \%$ & $2.12 \%$ \\
\hline 14 & $2.74 \%$ & $1.98 \%$ & $1.88 \%$ \\
\hline 15 & $2.99 \%$ & $1.98 \%$ & $1.70 \%$ \\
\hline 16 & $3.07 \%$ & $1.68 \%$ & $1.51 \%$ \\
\hline 17 & $2.52 \%$ & $1.42 \%$ & $1.17 \%$ \\
\hline 18 & $2.71 \%$ & $1.81 \%$ & $1.69 \%$ \\
\hline 19 & $2.71 \%$ & $2.36 \%$ & $2.33 \%$ \\
\hline 20 & $2.37 \%$ & $1.73 \%$ & $1.63 \%$ \\
\hline 21 & $2.06 \%$ & $1.56 \%$ & $1.37 \%$ \\
\hline 22 & $2.70 \%$ & $2.33 \%$ & $2.30 \%$ \\
\hline 23 & $2.21 \%$ & $1.76 \%$ & $1.71 \%$ \\
\hline 24 & $2.54 \%$ & $2.00 \%$ & $1.87 \%$ \\
\hline 25 & $2.37 \%$ & $1.99 \%$ & $1.91 \%$ \\
\hline 26 & $2.58 \%$ & $1.24 \%$ & $1.05 \%$ \\
\hline 27 & $2.56 \%$ & $1.86 \%$ & $1.66 \%$ \\
\hline 28 & $2.04 \%$ & $1.56 \%$ & $1.47 \%$ \\
\hline 29 & $1.90 \%$ & $1.52 \%$ & $1.49 \%$ \\
\hline 30 & $2.14 \%$ & $1.79 \%$ & $1.72 \%$ \\
\hline
\end{tabular}

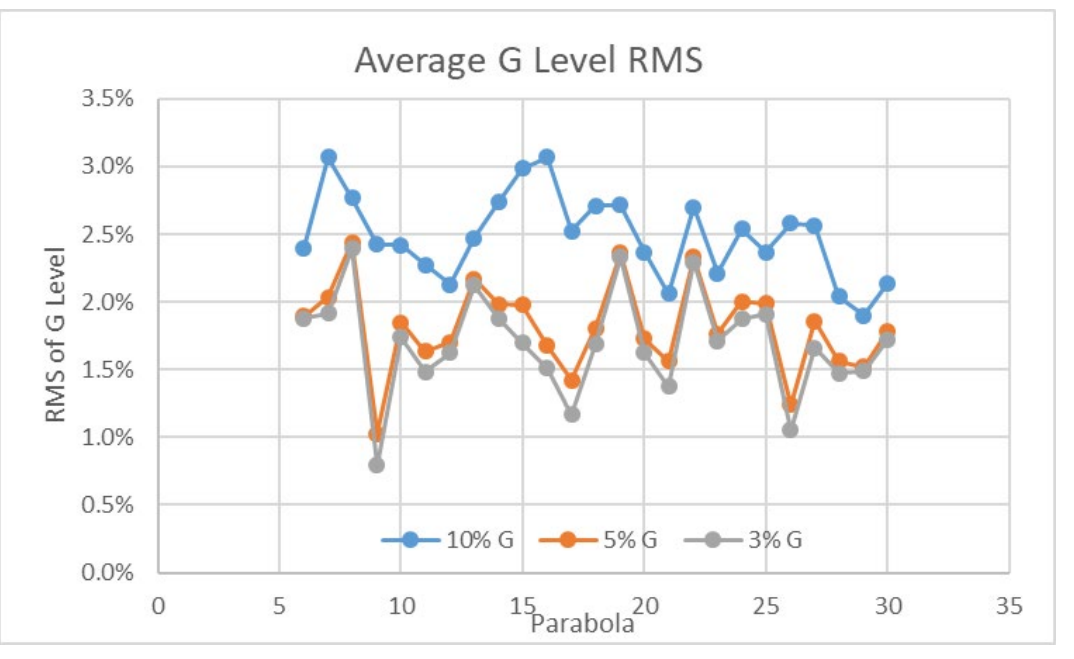




\begin{tabular}{|c|c|c|c|}
\hline \multicolumn{4}{|c|}{ Microgravity Period Length for Each Parabola } \\
\hline & \multicolumn{3}{|c|}{ Time Microgravity } \\
\hline Parabola \#: & $10 \%$ G & $5 \%$ G & $3 \%$ G \\
\hline 6 & 16.851 & 15.735 & 15.286 \\
\hline 7 & 19.46 & 16.675 & 16.073 \\
\hline 8 & 19.989 & 19.255 & 18.909 \\
\hline 9 & 20.388 & 17.825 & 17.345 \\
\hline 10 & 19.704 & 18.846 & 18.401 \\
\hline 11 & 19.395 & 18.659 & 18.18 \\
\hline 12 & 19.381 & 18.868 & 18.574 \\
\hline 13 & 18.635 & 18.119 & 17.766 \\
\hline 14 & 18.174 & 16.927 & 16.386 \\
\hline 15 & 19.763 & 17.457 & 16.788 \\
\hline 16 & 18.961 & 16.57 & 16.124 \\
\hline 17 & 18.987 & 17.17 & 16.691 \\
\hline 18 & 20.428 & 18.54 & 18.154 \\
\hline 19 & 19.237 & 18.565 & 18.278 \\
\hline 20 & 18.119 & 17.263 & 16.879 \\
\hline 21 & 20.241 & 19.657 & 18.975 \\
\hline 22 & 20.187 & 19.454 & 18.95 \\
\hline 23 & 20.558 & 19.985 & 19.761 \\
\hline 24 & 19.841 & 19.077 & 18.504 \\
\hline 25 & 21.246 & 20.637 & 20.16 \\
\hline 26 & 19.76 & 17.185 & 16.614 \\
\hline 27 & 19.628 & 18.043 & 17.439 \\
\hline 28 & 20.699 & 20.222 & 19.966 \\
\hline 29 & 20.486 & 19.786 & 19.413 \\
\hline 30 & 21.674 & 21.224 & 21.035 \\
\hline
\end{tabular}

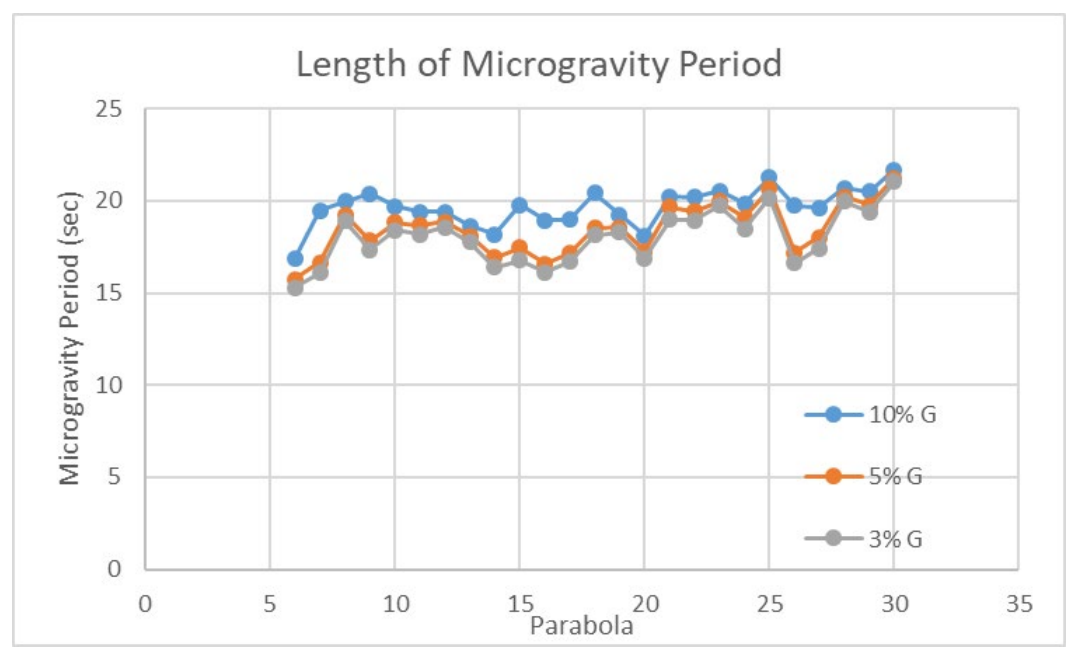




\section{Appendix C: Hand Soldering Visual Inspection}

\section{Visual Inspection Grading}

Flight samples

\begin{tabular}{|c|c|}
\hline & Key \\
\hline & no joint \\
\hline 1 & defective \\
2 & acceptable \\
3 & good \\
\hline
\end{tabular}




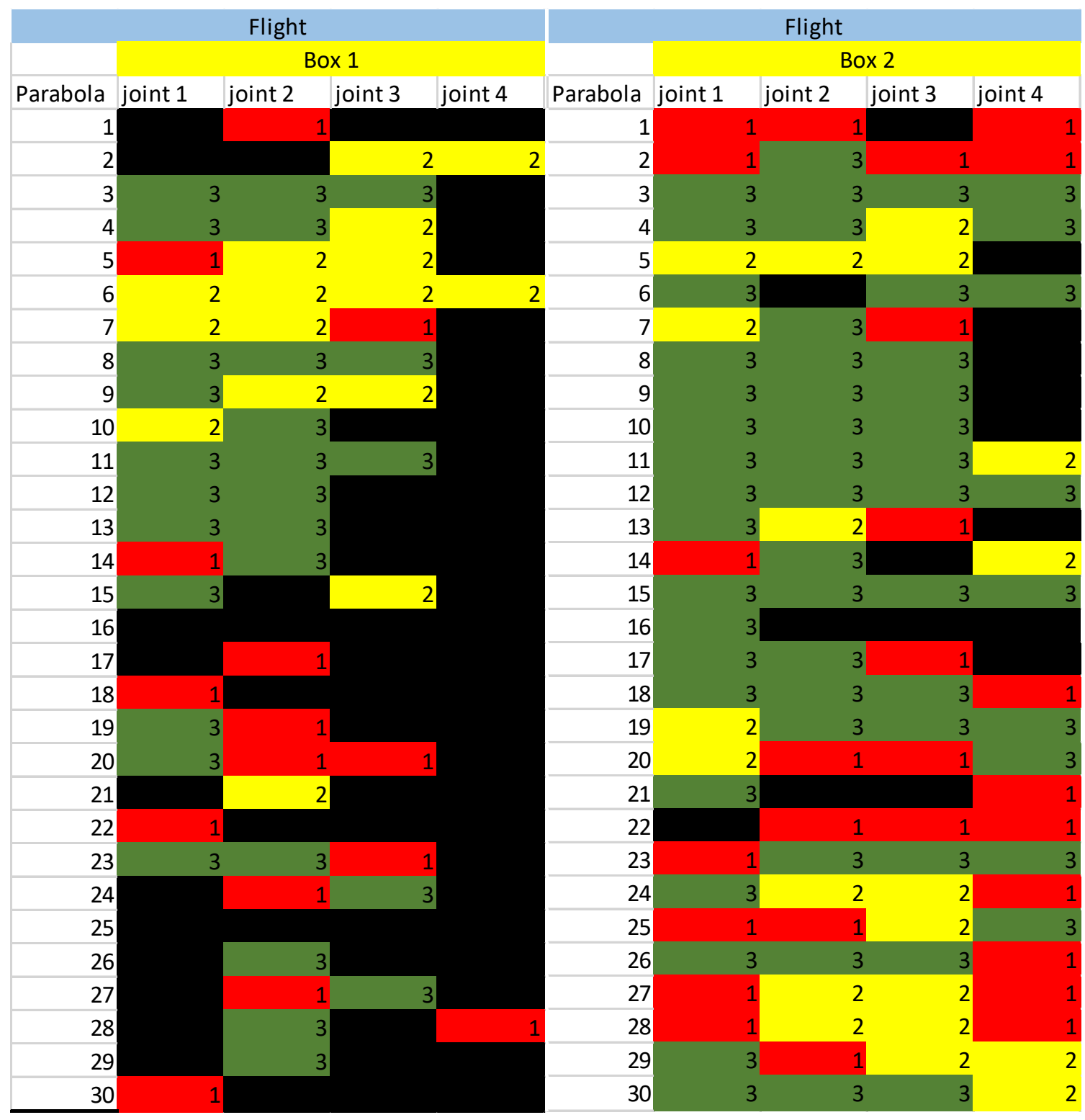




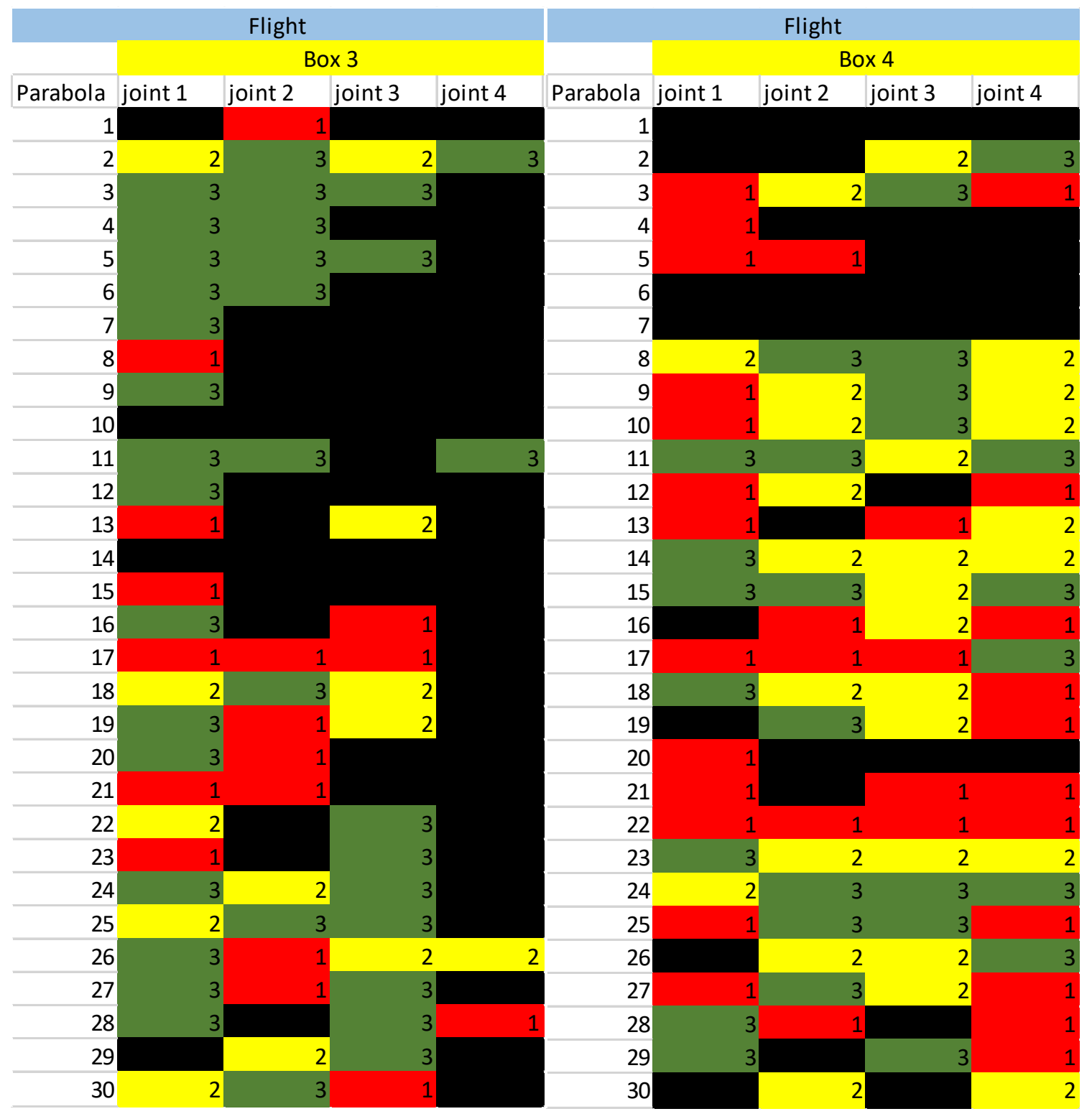


Ground samples 


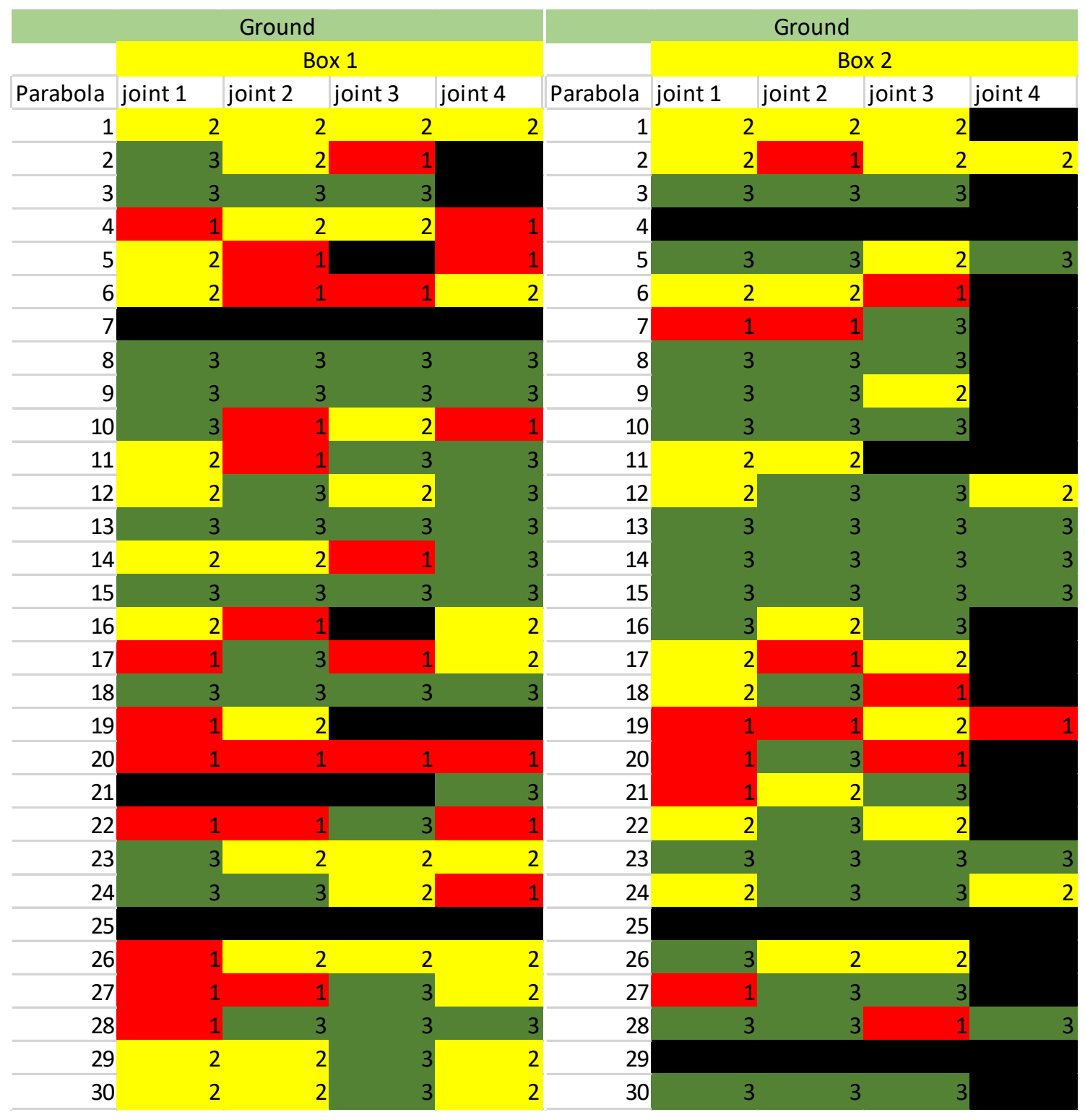




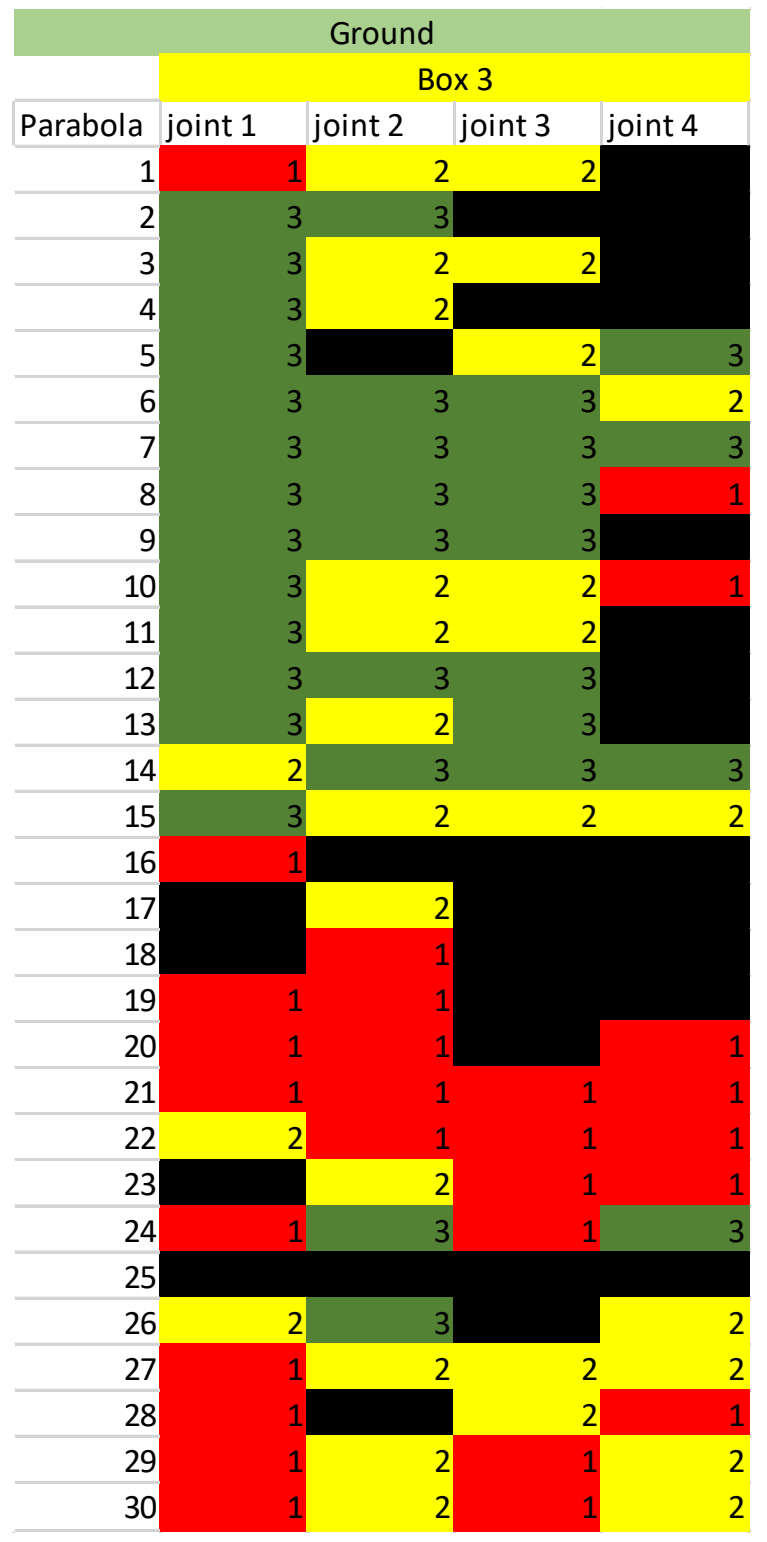




\section{Hand Soldering Defect Log}

Flight samples defects

\begin{tabular}{|l|l|}
\hline \multicolumn{1}{|c|}{ Defect } & \multicolumn{1}{c|}{ Defects and their Descriptions } \\
\hline fill & The joint did not fill the entirety of the through hole \\
\hline contact angle & Solder balled on the surface \\
\hline projection & Spike like feature on the surface of the solder joint \\
\hline bridged holes & The solder flow into an adjacent hole as well as the intended hole \\
\hline surface void & The joints surface had a cavity \\
\hline wet angle & An area of the through hole or surface pad was not wetted by the solder \\
\hline wetting & Solder did not wet the wire and/or through hole \\
\hline through hole & The joint had a continuous hole through the entire joint \\
\hline climbed wire & Solder wicked up the wire further than is acceptable \\
\hline wrong hole & Solder melted into a hole without a resistor \\
\hline surface spread & Solder spread on the board beyond the soldering pad \\
\hline
\end{tabular}




\begin{tabular}{|c|c|c|c|c|c|}
\hline \multicolumn{6}{|c|}{ Flight } \\
\hline \multirow[b]{2}{*}{ Parabola } & \multicolumn{5}{|c|}{ Box 1} \\
\hline & joint 1 & joint 2 & & joint 3 & joint 4 \\
\hline 1 & & fill & & & \\
\hline 2 & & & & & \\
\hline 3 & & & & & \\
\hline 4 & & & & & \\
\hline 5 & fill & & & & \\
\hline 6 & & & & & \\
\hline 7 & & & & & \\
\hline 8 & & & & & \\
\hline 9 & & & & & \\
\hline 10 & & & & & \\
\hline 11 & & & & & \\
\hline 12 & & & & & \\
\hline 13 & & & & & \\
\hline 14 & bridged holes & & & & \\
\hline 15 & & & & & \\
\hline 16 & & & & & \\
\hline 17 & & contact angle & & & \\
\hline 18 & fill & & & & \\
\hline 19 & & fill & & & \\
\hline 20 & & & & & \\
\hline 21 & & & & & \\
\hline 22 & fill & & & & \\
\hline 23 & & & fill & contact angle & \\
\hline 24 & & projection & & & \\
\hline 25 & & fill & & & \\
\hline 26 & & & & & \\
\hline 27 & & & & & \\
\hline 28 & & & & & projection \\
\hline 29 & & & & & \\
\hline 30 & fill & & & & \\
\hline
\end{tabular}




\begin{tabular}{|c|c|c|c|c|c|c|c|}
\hline \multicolumn{8}{|c|}{ Flight } \\
\hline \multirow[b]{2}{*}{ Parabola } & \multicolumn{7}{|c|}{ Box 2} \\
\hline & \multicolumn{2}{|c|}{ joint 1} & \multicolumn{2}{|c|}{ joint 2} & \multicolumn{2}{|c|}{ joint 3} & \multirow{2}{*}{$\begin{array}{l}\text { joint 4 } \\
\text { fill }\end{array}$} \\
\hline & fill & & fill & & & & \\
\hline $2 \mid f$ & fill & & & & fill & & fill \\
\hline 3 & & & & & & & \\
\hline 4 & & & & & & & \\
\hline 5 & & & & & & & \\
\hline 6 & & & & & & & \\
\hline 7 & & & & & fill & & \\
\hline 8 & & & & & & & \\
\hline 9 & & & & & & & \\
\hline 10 & & & & & & & \\
\hline 11 & & & & & & & \\
\hline 12 & & & & & & & \\
\hline 13 & & & & & fill & & \\
\hline 14 & contact angle & & & & & & \\
\hline 15 & & & & & & & \\
\hline 16 & & & & & & & \\
\hline 17 & & & & & wet angle & wetting & \\
\hline 18 & & & & & & & surface void \\
\hline 19 & & & & & & & \\
\hline 20 & & & fill & & wet angle & & \\
\hline 21 & & & & & & & surface void \\
\hline 22 & & & fill & contact angle & & & fill \\
\hline 23 & surface void & & & & & & \\
\hline 24 & & & & & & & surface void \\
\hline 25 & & & & & & & \\
\hline 26 & & & & & & & \\
\hline $27 f$ & fill & & & & & & fill \\
\hline $28 t$ & through hole & wet angle & & & & & fill \\
\hline 29 & & & through hole & wet angle & & & \\
\hline 30 & & & & & & & \\
\hline
\end{tabular}




\begin{tabular}{|r|r|l|l|l|l|}
\hline \\
\hline Parabola joint 1
\end{tabular}




\begin{tabular}{|c|c|c|c|c|c|c|c|c|}
\hline \multicolumn{9}{|c|}{ Flight } \\
\hline \multicolumn{9}{|c|}{ Box 4} \\
\hline Parabola & \multicolumn{2}{|c|}{ joint 1} & \multicolumn{2}{|c|}{ joint 2} & \multicolumn{2}{|c|}{ joint 3} & \multicolumn{2}{|c|}{ joint 4} \\
\hline 1 & & & & & & & & \\
\hline 2 & & & & & & & & \\
\hline & fill & & & & & & projection & \\
\hline 4 & climbed wire & & & & & & & \\
\hline & fill & & through hole & wet angle & & & & \\
\hline 6 & & & & & & & & \\
\hline 7 & & & & & & & & \\
\hline 8 & & & & & & & & \\
\hline 9 & fill & & & & & & & \\
\hline 10 & & & & & & & & \\
\hline 11 & & & & & & & & \\
\hline 12 & projection & & & & & & projection & \\
\hline 13 & projection & & & & projection & & & \\
\hline 14 & & & & & & & & \\
\hline 15 & & & & & & & & \\
\hline 16 & & & fill & projection & & & through hole & \\
\hline 17 & projection & & wet angle & & wet angle & through hole & & \\
\hline 18 & & & & & & & bridged holes & climbed wire \\
\hline 19 & & & & & & & fill & contact angle \\
\hline 20 & fill & projection & & & & & & \\
\hline 21 & fill & surface spread & & & fill & climbed wire & fill & surface spread \\
\hline 22 & fill & & wet angle & through hole & wet angle & through hole & fill & surface spread \\
\hline 23 & & & & & & & & \\
\hline 24 & & & & & & & & \\
\hline 25 & projection & & & & & & projection & \\
\hline 26 & & & & & & & & \\
\hline 27 & wet angle & & & & & & projection & \\
\hline 28 & & fill & & & & & climbed wire & \\
\hline 29 & & & & & & & climbed wire & \\
\hline 30 & & & & & & & & \\
\hline
\end{tabular}


Ground samples defects

\begin{tabular}{|c|c|c|c|c|}
\hline \multicolumn{5}{|c|}{ Ground } \\
\hline \multirow[b]{2}{*}{ Parabola } & \multicolumn{4}{|c|}{ Box 1} \\
\hline & joint 1 & joint 2 & joint 3 & joint 4 \\
\hline 1 & & & & \\
\hline 2 & & & fill & \\
\hline 3 & & & & \\
\hline 4 & fill & & & fill \\
\hline 5 & & fill & & fill \\
\hline 6 & & wrong hole & wrong hole & \\
\hline 7 & & & & \\
\hline 8 & & & & \\
\hline 9 & & & & \\
\hline 10 & fill & & & through hole \\
\hline 11 & & through hole & & \\
\hline 12 & & & & \\
\hline 13 & & & & \\
\hline 14 & & & fill & \\
\hline 15 & & & & \\
\hline 16 & & fill & & \\
\hline 17 & wetting & & wetting & \\
\hline 18 & & & & \\
\hline 19 & fill & & & \\
\hline 20 & projection & contact angle & projection & projection \\
\hline 21 & & & & \\
\hline 22 & fill & projection & & projection \\
\hline 23 & & & & \\
\hline 24 & & & & surface void \\
\hline 25 & & & & \\
\hline 26 & through hole & & & \\
\hline 27 & projection & surface void & & \\
\hline 28 & bridged holes & & & \\
\hline 29 & & & & \\
\hline 30 & & & & \\
\hline
\end{tabular}




\begin{tabular}{|c|c|c|c|c|}
\hline \multicolumn{5}{|c|}{ Ground } \\
\hline \multirow[b]{2}{*}{ Parabola } & \multicolumn{4}{|c|}{ Box 2} \\
\hline & joint 1 & joint 2 & joint 3 & joint 4 \\
\hline 1 & & & & \\
\hline 2 & & fill & & \\
\hline 3 & & & & \\
\hline 4 & & & & \\
\hline 5 & & & & \\
\hline 6 & & & fill & \\
\hline 7 & fill & through hole & & \\
\hline 8 & & & & \\
\hline 9 & & & & \\
\hline 10 & & & & \\
\hline 11 & & & & \\
\hline 12 & & & & \\
\hline 13 & & & & \\
\hline 14 & & & & \\
\hline 15 & & & & \\
\hline 16 & & & & \\
\hline 17 & & fill & & \\
\hline 18 & & & bridged holes & \\
\hline 19 & projection & projection & & projection \\
\hline 20 & projection & & projection & \\
\hline 21 & projection & & & \\
\hline 22 & & & & \\
\hline 23 & & & & \\
\hline 24 & & & & \\
\hline 25 & & & & \\
\hline 26 & & & & \\
\hline 27 & climbed wire & & & \\
\hline 28 & & & & \\
\hline 29 & & & & \\
\hline 30 & & & & \\
\hline
\end{tabular}




\begin{tabular}{|c|c|c|c|c|c|c|c|c|}
\hline \multicolumn{9}{|c|}{ Ground } \\
\hline \multicolumn{9}{|c|}{ Box 3} \\
\hline Parabola & \multicolumn{2}{|c|}{ joint 1} & \multicolumn{2}{|c|}{ joint 2} & \multicolumn{2}{|c|}{ joint 3} & \multicolumn{2}{|c|}{ joint 4} \\
\hline 1 & wetting & & & & & & & \\
\hline 2 & & & & & & & & \\
\hline 3 & & & & & & & & \\
\hline 4 & & & & & & & & \\
\hline 5 & & & & & & & & \\
\hline 6 & & & & & & & & \\
\hline 7 & & & & & & & & \\
\hline 8 & & & & & & & fill & \\
\hline 9 & & & & & & & & \\
\hline 10 & & & & & & & fill & \\
\hline 11 & & & & & & & & \\
\hline 12 & & & & & & & & \\
\hline 13 & & & & & & & & \\
\hline 14 & & & & & & & & \\
\hline 15 & & & & & & & & \\
\hline 16 & fill & wetting & & & & & & \\
\hline 17 & & & & & & & & \\
\hline 18 & & fill & & & & & & \\
\hline 19 & & & projection & & fill & & & \\
\hline 20 & fill & climbed wire & fill & contact angle & & & fill & \\
\hline 21 & fill & & fill & & fill & & fill & \\
\hline 22 & & & fill & projection & fill & wetting & fill & projection \\
\hline 23 & & & & & fill & & fill & \\
\hline 24 & fill & projection & & & fill & & & \\
\hline 25 & & & & & & & & \\
\hline 26 & & & & & & & & \\
\hline 27 & projection & & & & & & & \\
\hline 28 & projection & & & & & & climbed wire & \\
\hline 29 & surface void & & & & fill & & & \\
\hline 30 & projection & & & & projection & & & \\
\hline
\end{tabular}




\section{Appendix D: Visual Comparison of Microscope Images vs Processed Images with Voidage Percentages}

The following is a sampling of joints showing their original microscope images, colored images used for image processing, and percent void. To give a visual representation of what voids look like in an image and the correlation between these voids and an overall voidage percent, these samples have been laid out in order of ascending voidage.

Porosity $=0.7528 \% \quad$ joint designation $=$ FP30B2j2
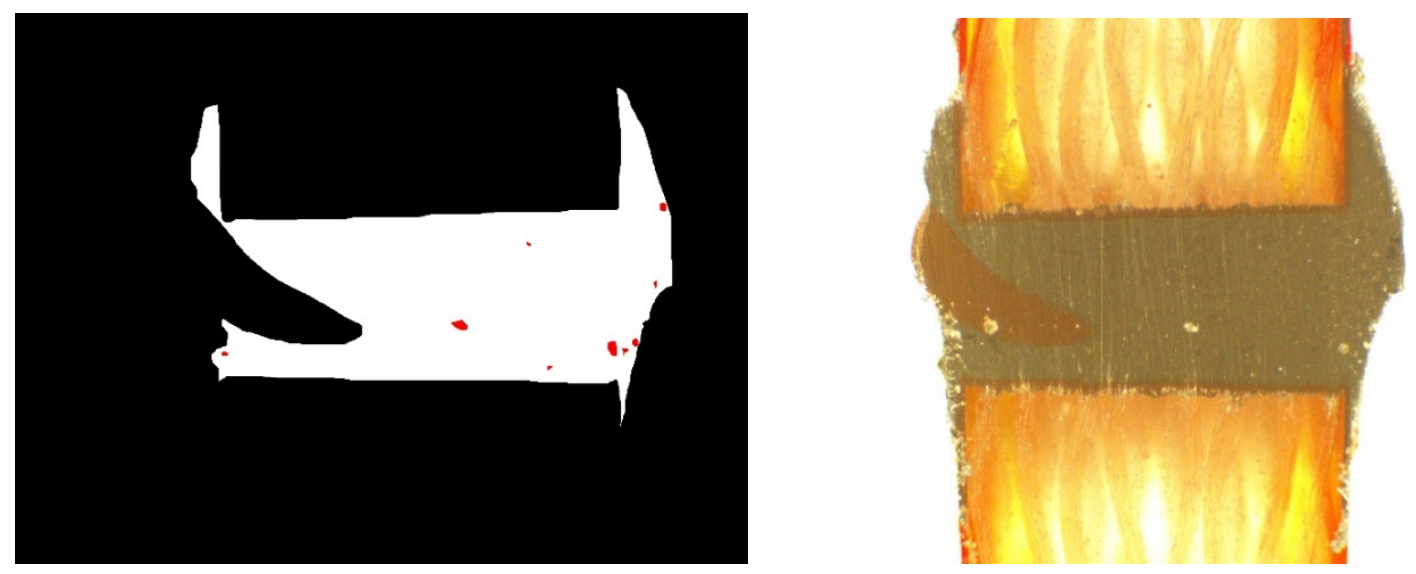

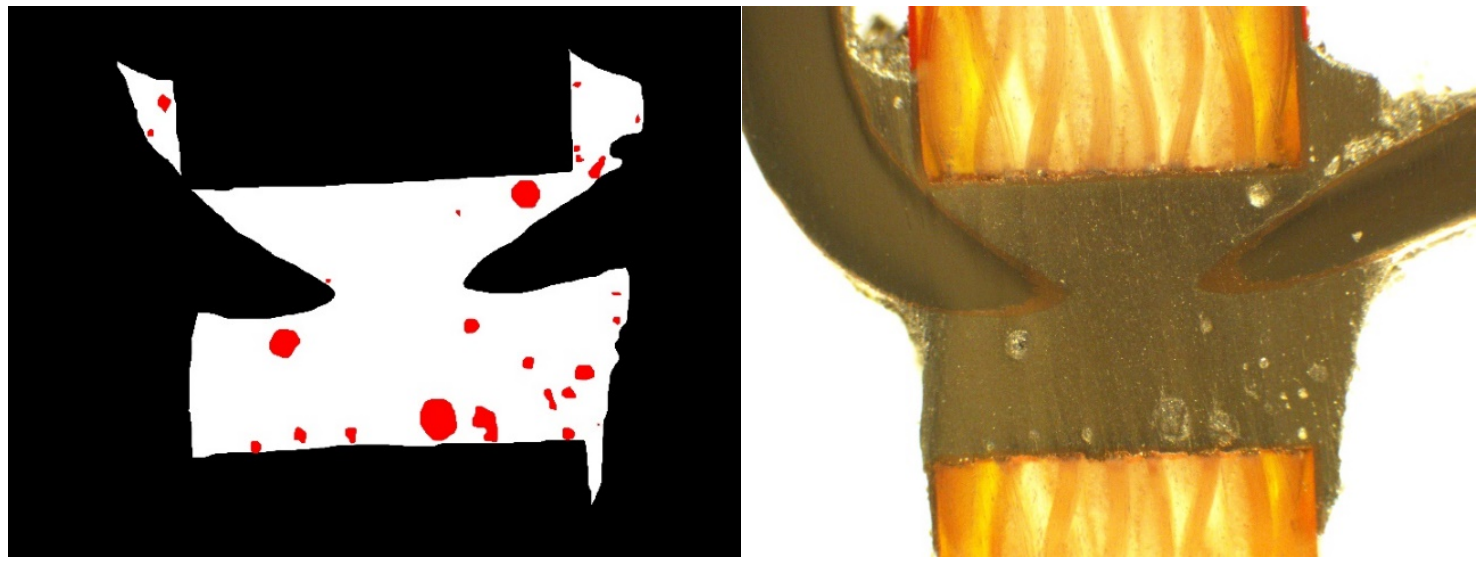

$1.5159 \%$

FP14B1j1
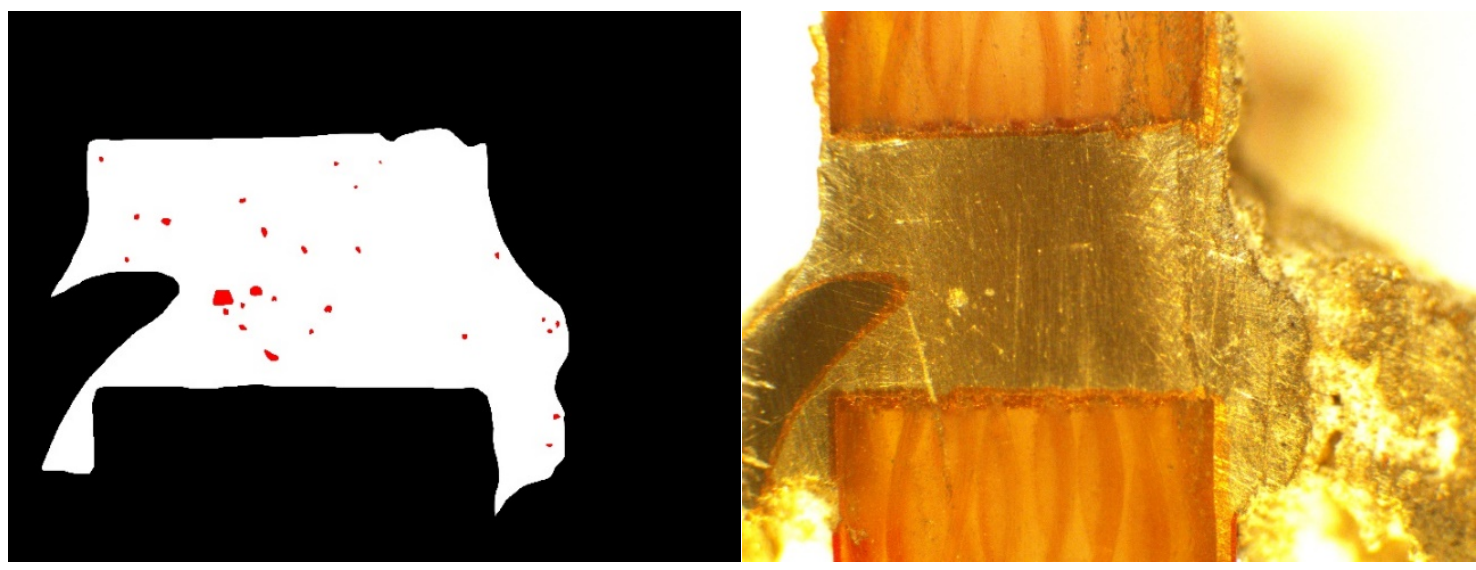

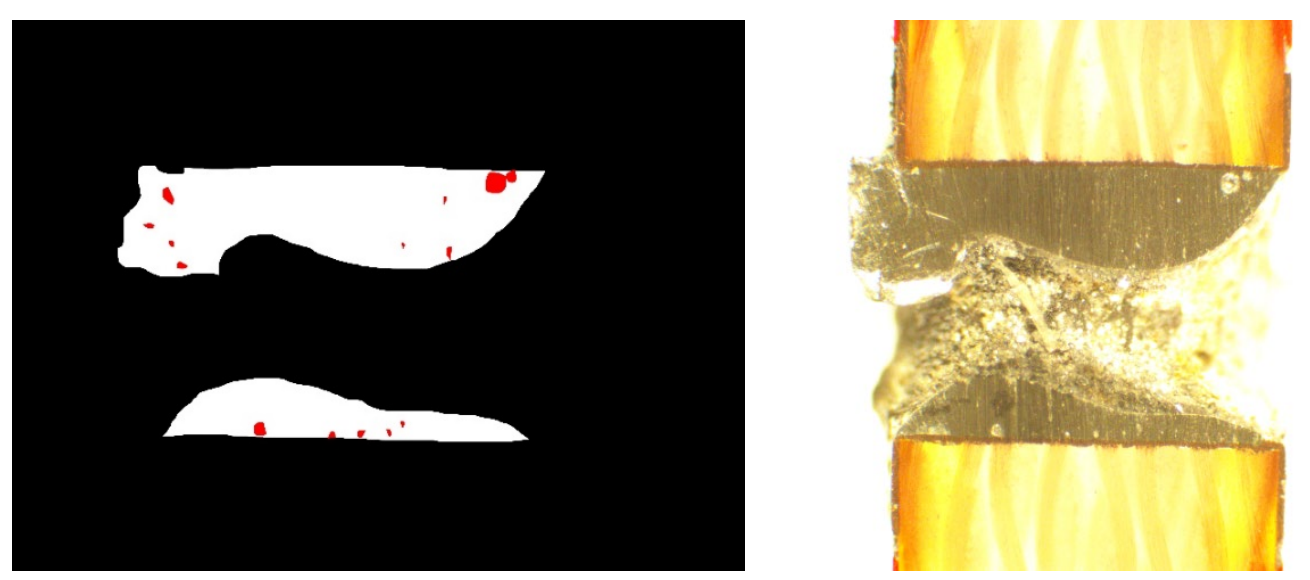

$2.7228 \%$

FP19B2j2
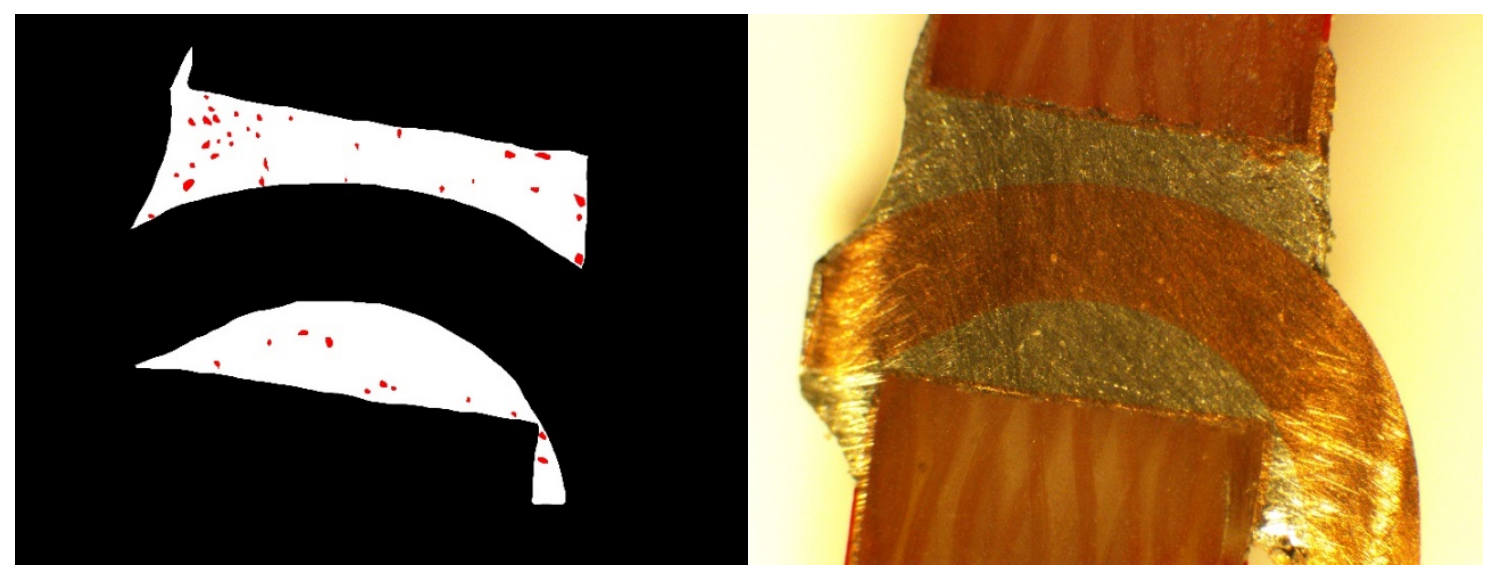
$2.7277 \% \quad$ FP13B1j2

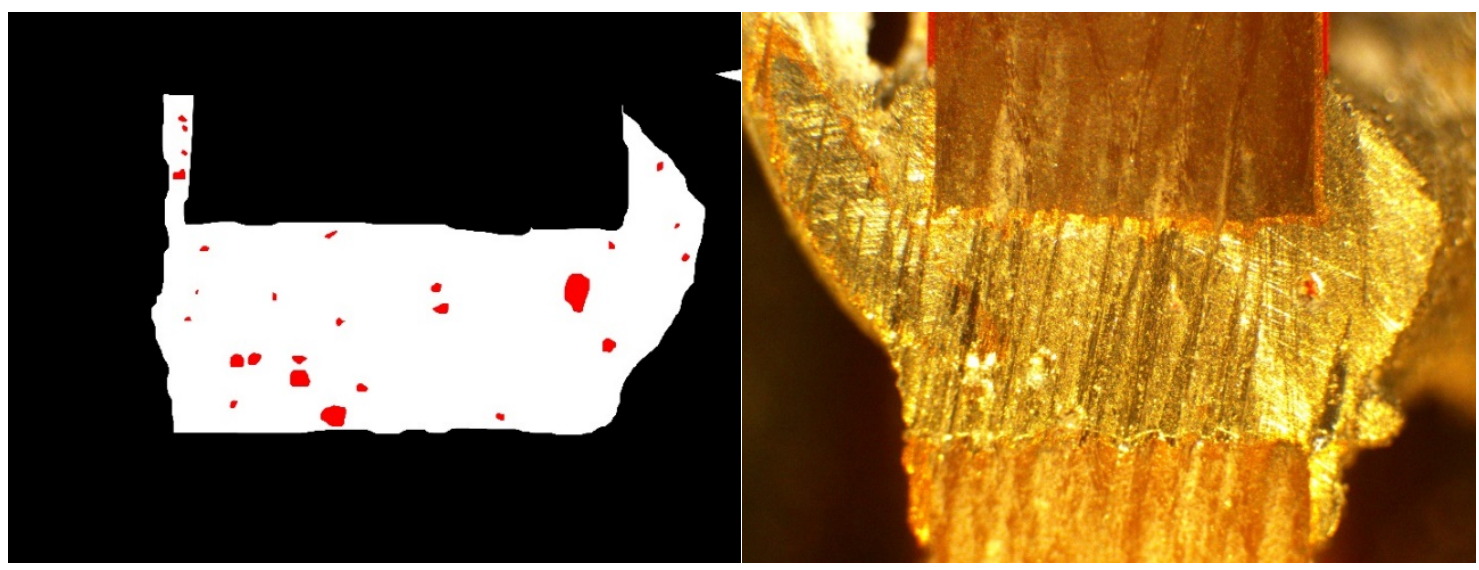

$3.2346 \% \quad F P 28 B 4 j 2$

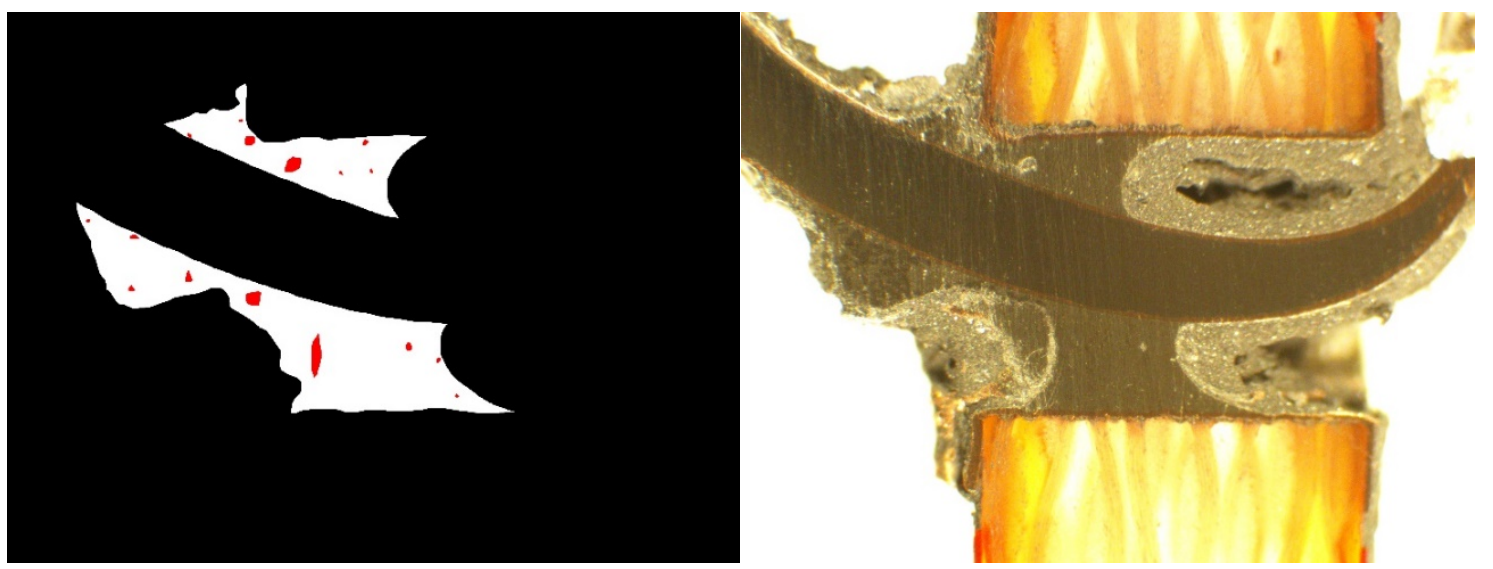




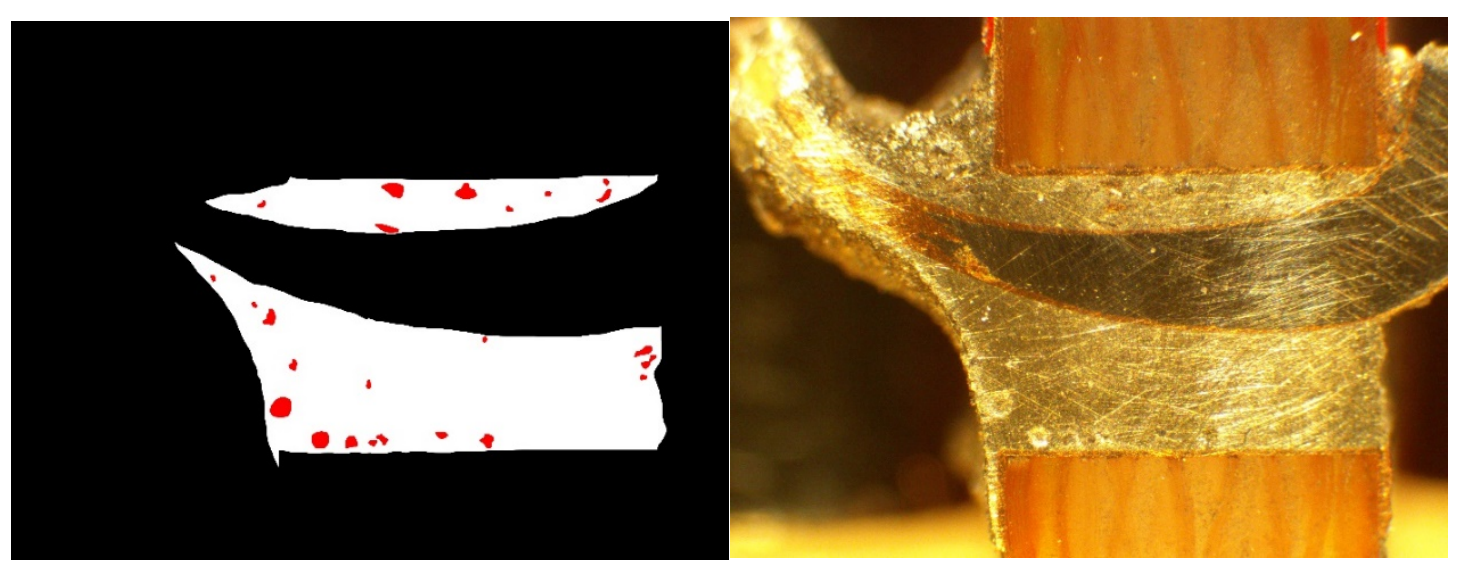

$3.9229 \%$

FP28B2j1
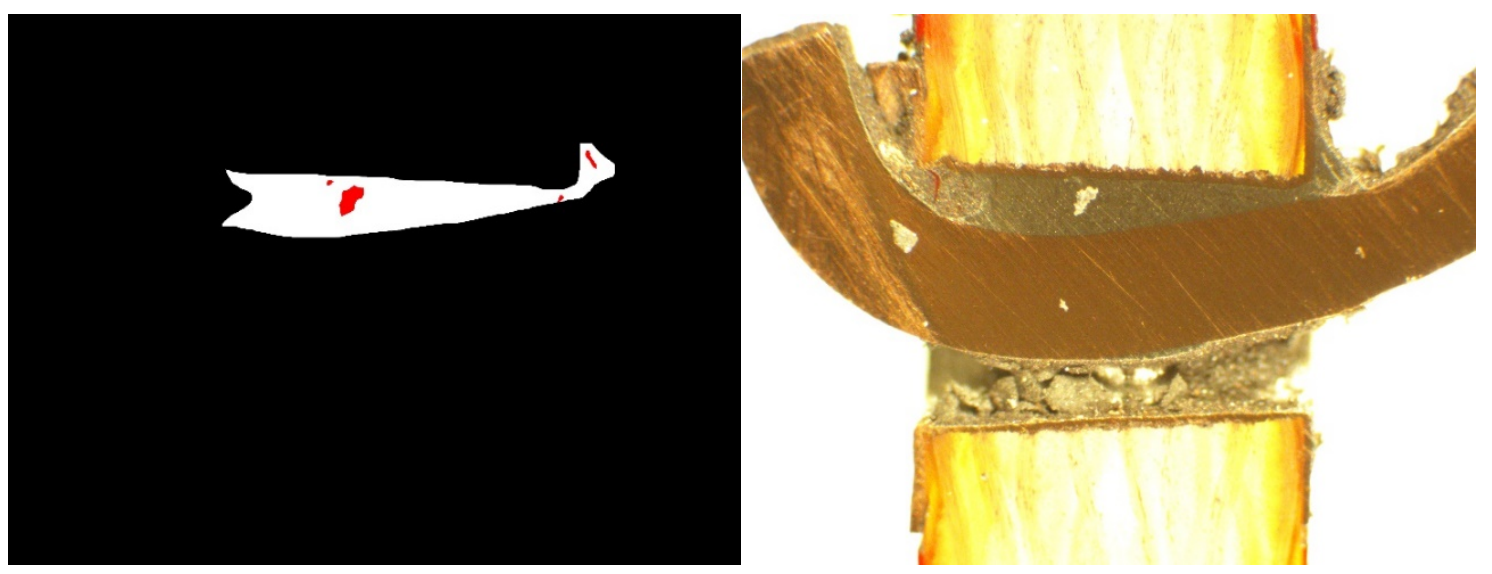

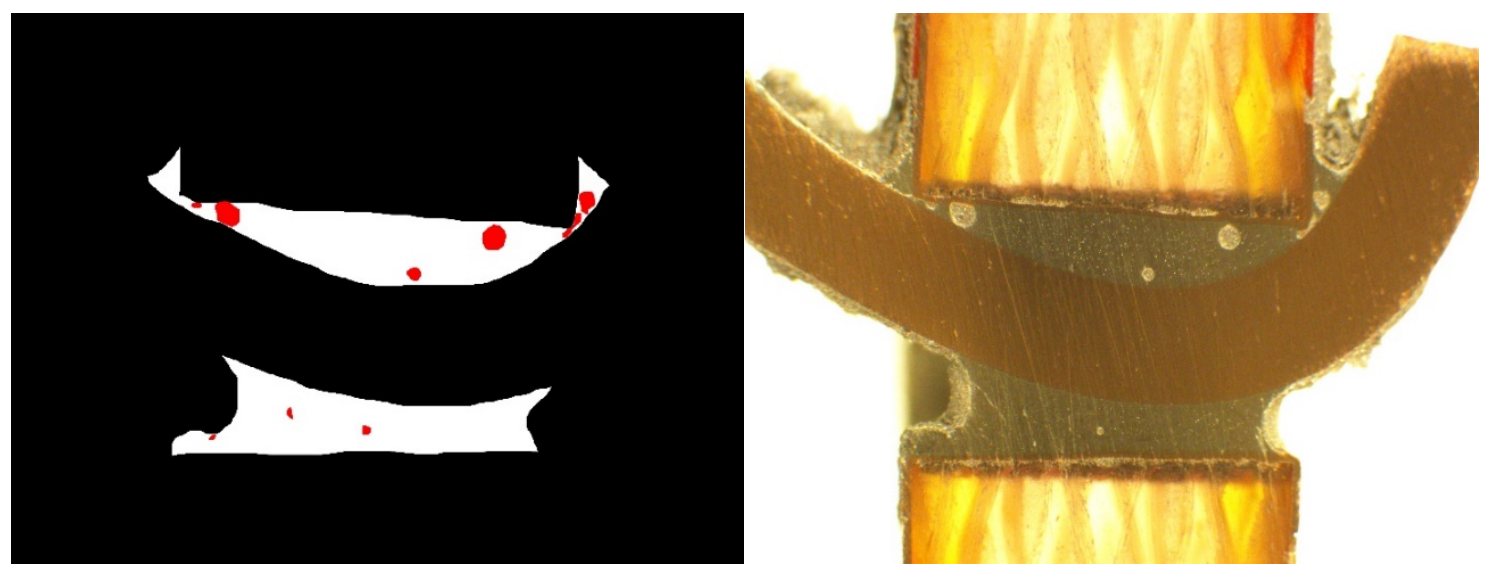

$5.42855 \% \quad F P 10 B 1 j 1$
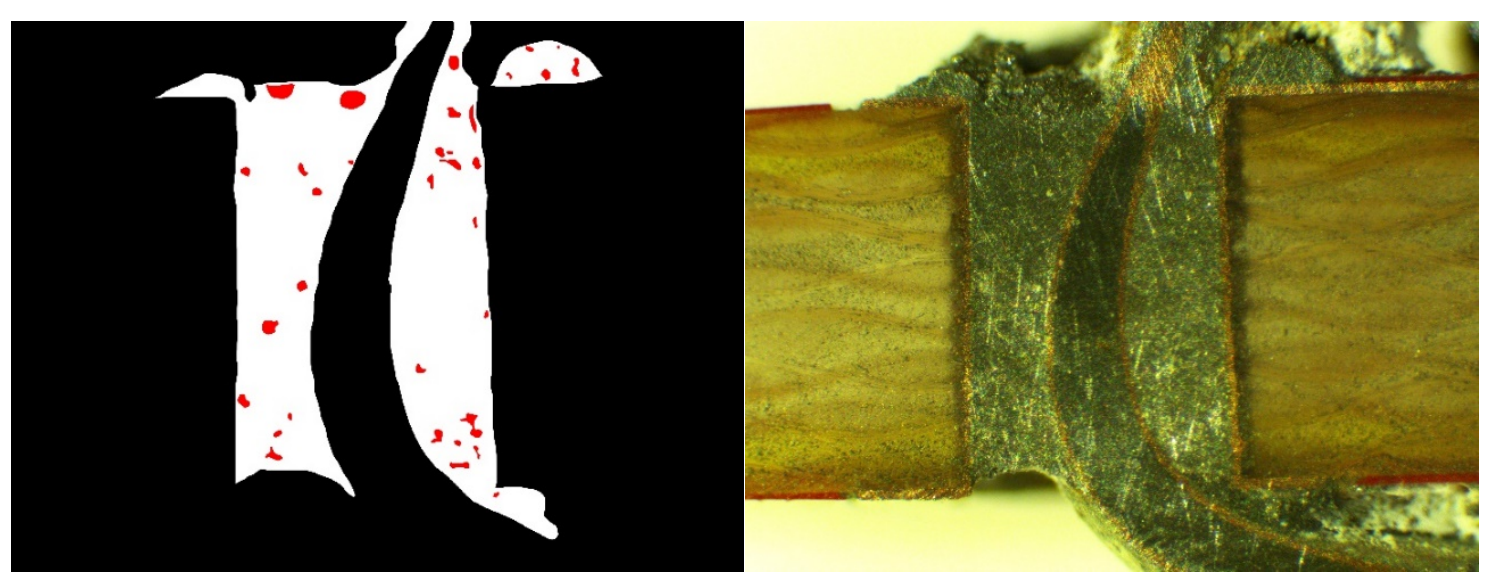

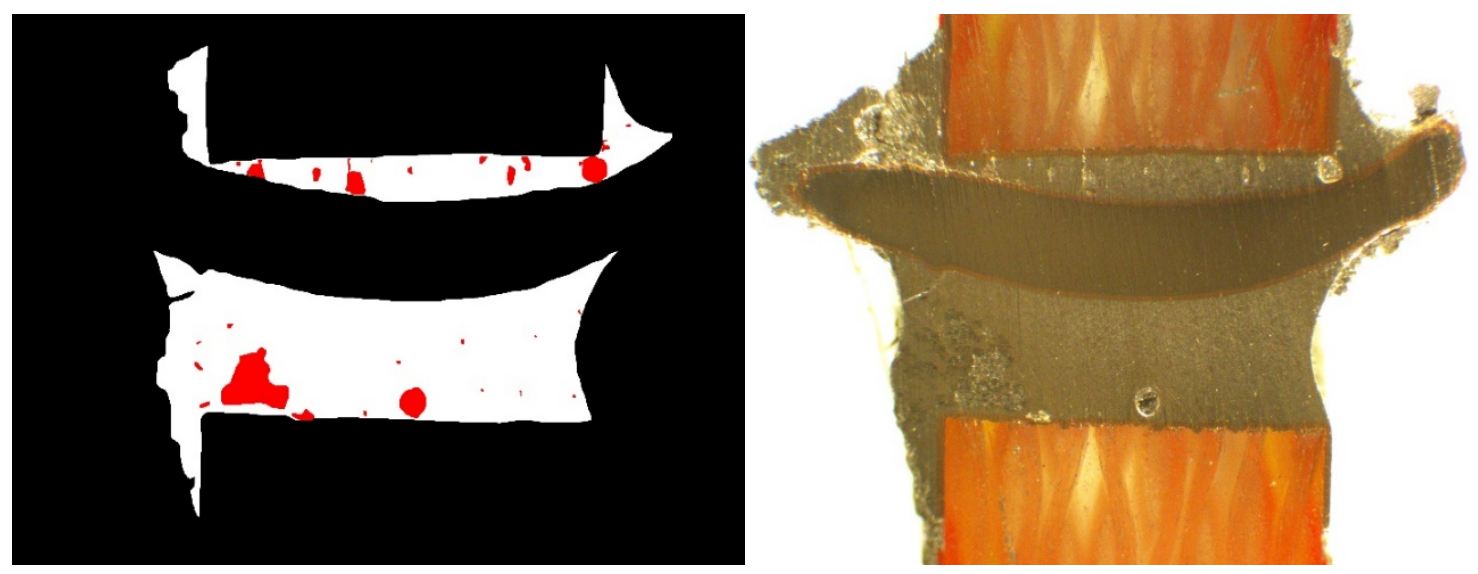

$18.2097 \% \quad$ FP28B4j1
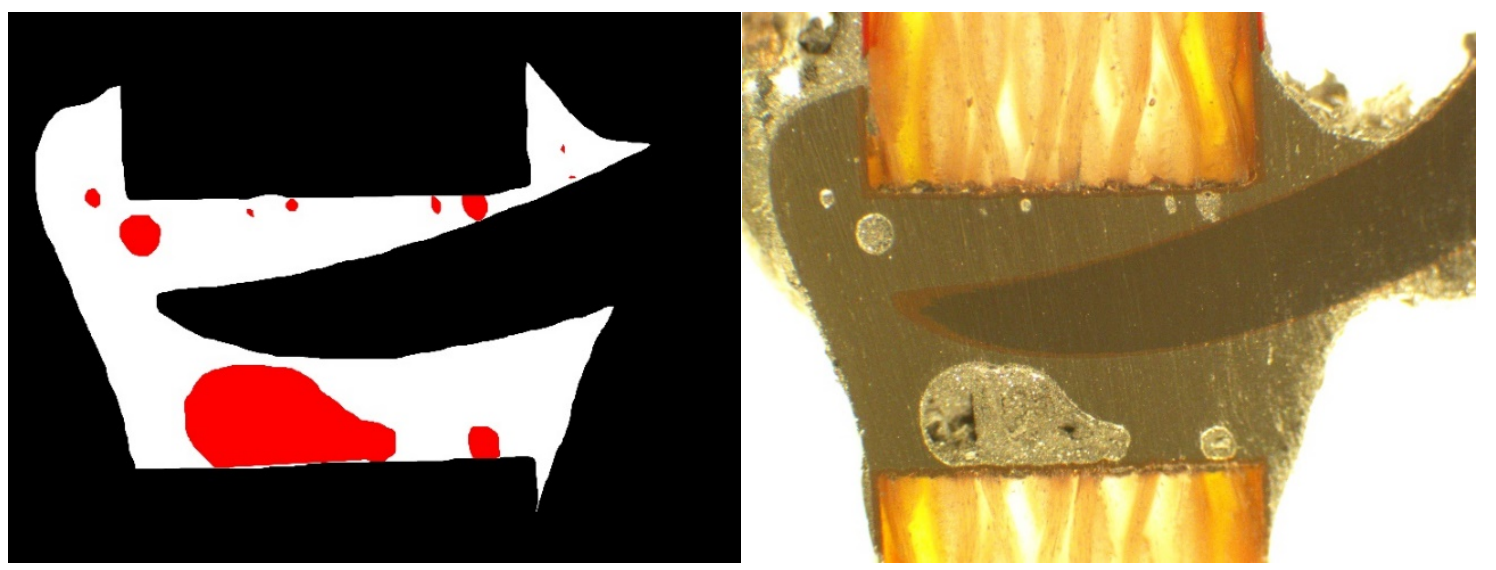
$22.9460 \% \quad F P 15 B 2 j 3$
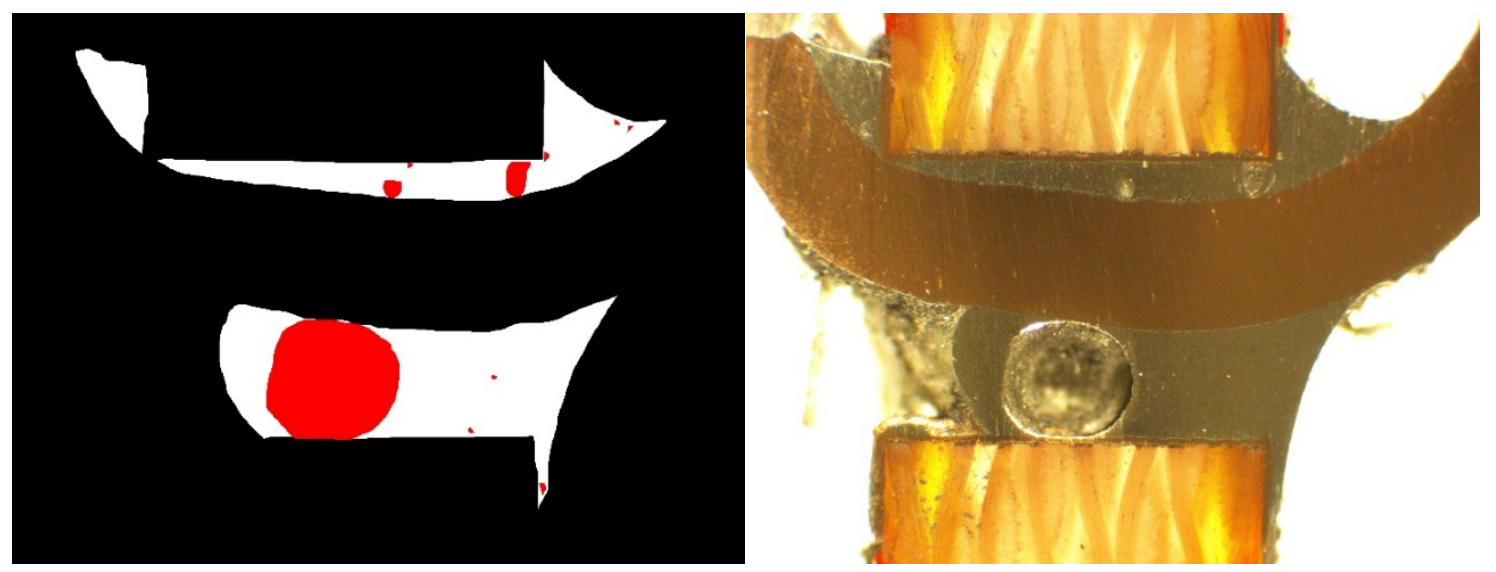

$32.3556 \% \quad$ FP10B1j2
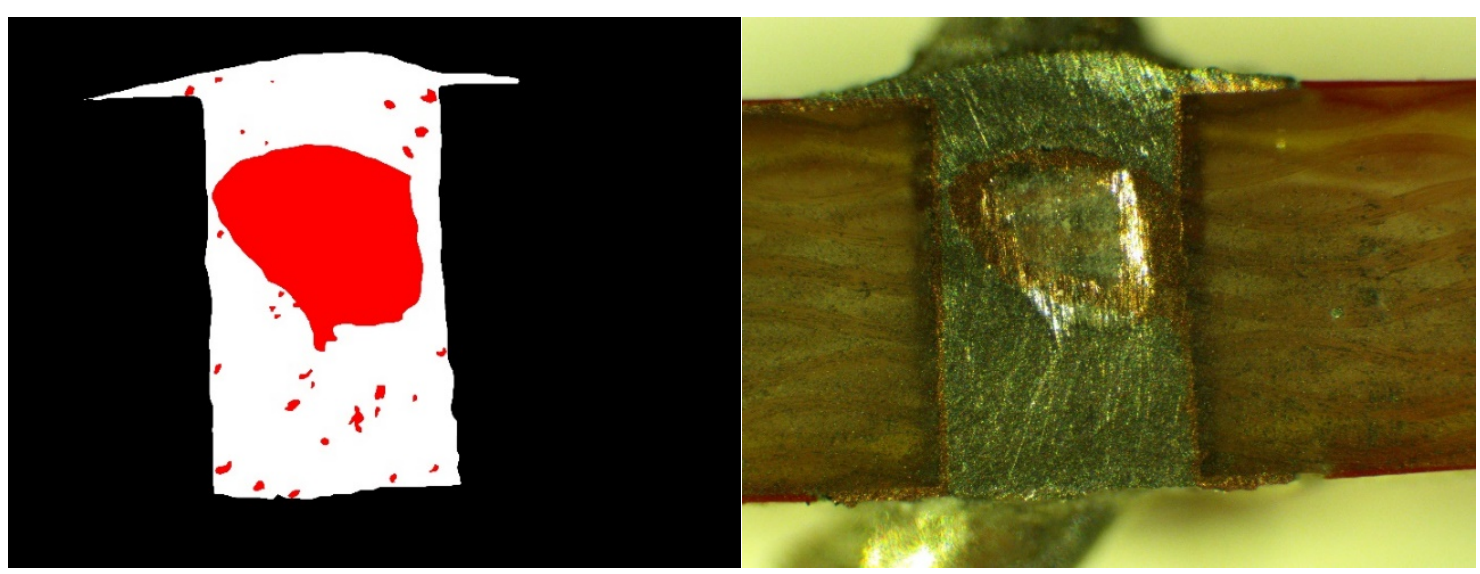


\section{Appendix E: Sample Porosity}

Flight sample porosity 


\begin{tabular}{|c|c|c|c|c|c|c|c|c|c|c|c|c|c|}
\hline \multicolumn{14}{|c|}{ Flight } \\
\hline \multicolumn{14}{|c|}{ Box 1} \\
\hline Parabola & Joint & & Colorization 1 & Colorization 2 & Colorization 3 & Average & Parabola & Joint & & Colorization 1 & Colorization 2 & Colorization 3 & Average \\
\hline \multirow[t]{4}{*}{1} & & $\overline{1}$ & & & & & 16 & & 1 & & & & \\
\hline & & 2 & & & & & & & 2 & & & & \\
\hline & & 3 & & & & & & & 3 & & & & \\
\hline & & 4 & & & & & & & 4 & & & & \\
\hline \multirow[t]{4}{*}{2} & & 1 & & & & & 17 & & 1 & & & & \\
\hline & & 2 & & & & & & & 2 & 1.3278 & & & 1.3278 \\
\hline & & 3 & & & & & & & 3 & & & & \\
\hline & & 4 & & & & & & & 4 & & & & \\
\hline \multirow[t]{4}{*}{3} & & 1 & & & & & 18 & & 1 & 2.032 & & & 2.032 \\
\hline & & 2 & & & & & & & 2 & & & & \\
\hline & & 3 & & & & & & & 3 & & & & \\
\hline & & 4 & & & & & & & 4 & & & & \\
\hline \multirow[t]{4}{*}{4} & & 1 & & & & & 19 & & 1 & 2.4946 & & & 2.4946 \\
\hline & & 2 & & & & & & & 2 & 1.2923 & & & 1.2923 \\
\hline & & 3 & & & & & & & 3 & & & & \\
\hline & & 4 & & & & & & & 4 & & & & \\
\hline \multirow[t]{4}{*}{5} & & 1 & & & & & 20 & & 1 & & & & \\
\hline & & 2 & & & & & & & 2 & & & & \\
\hline & & 3 & & & & & & & 3 & & & & \\
\hline & & 4 & & & & & & & 4 & & & & \\
\hline \multirow[t]{4}{*}{6} & & 1 & 3.5313 & 5.164 & & 4.34765 & 21 & & 1 & & & & \\
\hline & & 2 & 3.5813 & & & 3.5813 & & & 2 & 13.2706 & & & 13.2706 \\
\hline & & 3 & 2.1961 & & & 2.1961 & & & 3 & & & & \\
\hline & & 4 & 1.5612 & & & $\quad 1.5612$ & & & 4 & & & & \\
\hline \multirow[t]{4}{*}{7} & & 1 & 5.1635 & & & 5.1635 & 22 & & 1 & 1.2923 & & & 1.2923 \\
\hline & & 2 & 21.2104 & & & 21.2104 & & & 2 & & & & \\
\hline & & 3 & 1.8366 & & & 1.8366 & & & 3 & & & & \\
\hline & & 4 & & & & & & & 4 & & & & \\
\hline \multirow[t]{4}{*}{8} & & 1 & 8.5343 & & & 8.5343 & 23 & & 1 & 5.4788 & & & 5.4788 \\
\hline & & 2 & 1.9547 & & & 1.9547 & & & 2 & & & & \\
\hline & & 3 & 5.7641 & & & 5.7641 & & & 3 & & & & \\
\hline & & 4 & & & & & & & 4 & & & & \\
\hline \multirow[t]{4}{*}{9} & & 1 & 8.7643 & 7.007 & & 7.88565 & 24 & & 1 & & & & \\
\hline & & 2 & 2.8121 & 4.26 & & 3.53605 & & & 2 & 14.7379 & & & 14.7379 \\
\hline & & 3 & 4.6027 & 5.175 & & 4.88885 & & & 3 & & & & \\
\hline & & 4 & & & & & & & 4 & & & & \\
\hline 10 & & 1 & 3.9281 & 6.929 & & 5.42855 & 25 & & 1 & & & & \\
\hline & & 2 & 29.2792 & 35.432 & & 32.3556 & & & 2 & & & & \\
\hline & & 3 & & & & & & & 3 & & & & \\
\hline & & 4 & & & & & & & 4 & & & & \\
\hline 11 & & 1 & 2.9526 & 9.853 & 6.701 & 6.5022 & 26 & & 1 & & & & \\
\hline & & 2 & 3.8926 & 11.355 & 4.897 & $6.714867 \mid$ & & & 2 & & & & \\
\hline & & 3 & 4.8025 & 17.042 & & 10.92225 & & & 3 & & & & \\
\hline & & 4 & & & & & & & 4 & & & & \\
\hline 12 & & 1 & & & & & 27 & & 1 & & & & \\
\hline & & 2 & & & & & & & 2 & & & & \\
\hline & & 3 & & & & & & & 3 & & & & \\
\hline & & 4 & & & & & & & 4 & & & & \\
\hline 13 & & 1 & 1.0204 & & & 1.0204 & 28 & & 1 & & & & \\
\hline & & 2 & 2.7277 & & & 2.7277 & & & 2 & 1.3215 & & & 1.3215 \\
\hline & & 3 & & & & & & & 3 & & & & \\
\hline & & 4 & & & & & & & 4 & 2.0468 & & & 2.0468 \\
\hline 14 & & 1 & 1.5159 & & & 1.5159 & 29 & & 1 & & & & \\
\hline & & 2 & 1.027 & & & 1.027 & & & 2 & 3.0573 & 5.463 & & 4.26015 \\
\hline & & 3 & & & & & & & 3 & & & & \\
\hline & & 4 & & & & & & & 4 & & & & \\
\hline 15 & & 1 & 2.9773 & & & 2.9773 & 30 & & 1 & 2.3601 & & & 2.3601 \\
\hline & & 2 & & & & & & & 2 & & & & \\
\hline & & 3 & 1.0497 & & & 1.0497 & & & 3 & & & & \\
\hline & & 4 & & & & & & & 4 & & & & \\
\hline
\end{tabular}




\begin{tabular}{|c|c|c|c|c|c|c|c|c|c|c|c|c|}
\hline \multicolumn{13}{|c|}{ Flight } \\
\hline \multicolumn{13}{|c|}{ Box 2} \\
\hline Parabola & Joint & & Colorization 1 & Colorization 2 & Colorization 3 & Average & Parabola & Joint & Colorization 1 & Colorization 2 & Colorization 3 & Average \\
\hline \multirow[t]{4}{*}{1} & & 1 & & & & & 16 & 1 & 0.8115 & & & 0.8115 \\
\hline & & 2 & & & & & & 2 & & & & \\
\hline & & 3 & & & & & & 3 & & & & \\
\hline & & 4 & & & & & & 4 & & & & \\
\hline \multirow[t]{4}{*}{2} & & 1 & & & & & 17 & 1 & 1.0937 & & & 1.0937 \\
\hline & & 2 & & & & & & 2 & 21.352 & & & 21.352 \\
\hline & & 3 & & & & & & 3 & 6.0699 & & & 6.0699 \\
\hline & & 4 & & & & & & 4 & & & & \\
\hline \multirow[t]{4}{*}{3} & & 1 & & & & & 18 & 1 & 4.2514 & & & 4.2514 \\
\hline & & 2 & & & & & & 2 & 3.3092 & & & 3.3092 \\
\hline & & 3 & & & & & & 3 & 14.68 & & & 14.68 \\
\hline & & 4 & & & & & & 4 & 4.24 & & & 4.24 \\
\hline \multirow[t]{4}{*}{4} & & 1 & & & & & 19 & 1 & 1.7219 & & & 1.7219 \\
\hline & & 2 & & & & & & 2 & 2.7228 & & & 2.7228 \\
\hline & & 3 & & & & & & 3 & 4.0007 & & & 4.0007 \\
\hline & & 4 & & & & & & 4 & & & & \\
\hline \multirow[t]{4}{*}{5} & & 1 & 1.0914 & & & 1.0914 & 20 & 1 & & & & \\
\hline & & 2 & 0.8045 & & & 0.8045 & & 2 & & & & \\
\hline & & 3 & 1.3972 & & & 1.3972 & & 3 & & & & \\
\hline & & 4 & & & & & & 4 & & & & \\
\hline \multirow[t]{4}{*}{$\overline{6}$} & & 1 & & & & & 21 & 1 & 0.9053 & & & 0.9053 \\
\hline & & 2 & & & & & & 2 & & & & \\
\hline & & 3 & 6.8237 & & & 6.8237 & & 3 & & & & \\
\hline & & 4 & 3.1284 & & & 3.1284 & & 4 & 8.2472 & & & 8.2472 \\
\hline \multirow[t]{4}{*}{7} & & 1 & 14.7668 & & & 14.7668 & 22 & 1 & & & & \\
\hline & & 2 & 6.7774 & & & 6.7774 & & 2 & & & & \\
\hline & & 3 & 2.7787 & & & 2.7787 & & 3 & & & & \\
\hline & & 4 & & & & & & 4 & & & & \\
\hline \multirow[t]{4}{*}{8} & & 1 & 16.2247 & & & 16.2247 & 23 & 1 & 21.27 & & & 21.27 \\
\hline & & 2 & 1.9791 & & & 1.9791 & & 2 & 13.454 & & & 13.454 \\
\hline & & 3 & 9.4981 & & & 9.4981 & & 3 & 0.2222 & & & 0.2222 \\
\hline & & 4 & & & & & & 4 & & & & \\
\hline \multirow[t]{4}{*}{9} & & 1 & 3.9407 & 8.194 & & 6.06735 & 24 & 1 & 2.6108 & & & 2.6108 \\
\hline & & 2 & 5.084 & 6.082 & & 5.583 & & 2 & 2.0857 & & & 2.0857 \\
\hline & & 3 & 3.5694 & 11.135 & & 7.3522 & & 3 & 22.24 & & & 22.24 \\
\hline & & 4 & & & & & & 4 & 3.7815 & & & 3.7815 \\
\hline 10 & & 1 & 2.7063 & 4.936 & & 3.82115 & 25 & 1 & 24.439 & & & 24.439 \\
\hline & & 2 & 3.8406 & 5.596 & & 4.7183 & & 2 & 4.4005 & & & 4.4005 \\
\hline & & 3 & 21.538 & 25.406 & & 23.472 & & 3 & 15.223 & & & 15.223 \\
\hline & & 4 & & & & & & 4 & 15.772 & & & 15.772 \\
\hline 11 & & 1 & & & & & 26 & 1 & 17.631 & & & 17.631 \\
\hline & & 2 & 5.0174 & & & 5.0174 & & 2 & 7.889 & & & 7.889 \\
\hline & & 3 & 2.6937 & 9.898 & & 6.29585 & & 3 & 27.324 & & & 27.324 \\
\hline & & 4 & 4.2211 & 8.491 & & 6.35605 & & 4 & 14.077 & & & 14.077 \\
\hline 12 & & 1 & 2.9723 & & & 2.9723 & 27 & 1 & 4.6059 & & & 4.6059 \\
\hline & & 2 & 6.8322 & & & 6.8322 & & 2 & 3.1923 & & & 3.1923 \\
\hline & & 3 & & & & & & 3 & 44.693 & & & 44.693 \\
\hline & & 4 & & & & & & 4 & & & & \\
\hline 13 & & 1 & 5.4476 & & & 5.4476 & 28 & 1 & 3.9229 & & & 3.9229 \\
\hline & & 2 & 1.4515 & & & 1.4515 & & 2 & 13.889 & & & 13.889 \\
\hline & & 3 & 1.7438 & & & 1.7438 & & 3 & 2.4767 & & & 2.4767 \\
\hline & & 4 & & & & & & 4 & 1.6116 & & & 1.6116 \\
\hline 14 & & 1 & 5.186 & & & 5.186 & 29 & 1 & 2.0272 & 9.646 & & 5.8366 \\
\hline & & 2 & 1.1055 & & & 1.1055 & & 2 & 3.9269 & & & 3.9269 \\
\hline & & 3 & & & & & & 3 & 2.9311 & 8.067 & & 5.49905 \\
\hline & & 4 & 1.298 & & & 1.298 & & 4 & 5.3552 & & & 5.3552 \\
\hline 15 & & 1 & 5.6803 & & & 5.6803 & 30 & 1 & 21.557 & & & 21.557 \\
\hline & & 2 & 18.572 & & & 18.572 & & 2 & 0.7528 & & & 0.7528 \\
\hline & & 3 & 22.946 & & & 22.946 & & 3 & 3.5511 & & & 3.5511 \\
\hline & & 4 & 30.193 & & & 30.193 & & 4 & 5.6806 & & & 5.6806 \\
\hline
\end{tabular}




\begin{tabular}{|c|c|c|c|c|c|c|c|c|c|c|c|c|}
\hline \multicolumn{13}{|c|}{ Flight } \\
\hline \multicolumn{13}{|c|}{ Box 3} \\
\hline Parabola & Joint & & Colorization 1 & Colorization 2 & Colorization 3 & Average & Parabola & Joint & Colorization 1 & Colorization 2 & Colorization 3 & Average \\
\hline \multirow[t]{4}{*}{1} & & 1 & & & & & 16 & 1 & & & & \\
\hline & & 2 & & & & & & 2 & & & & \\
\hline & & 3 & & & & & & 3 & & & & \\
\hline & & 4 & & & & & & 4 & & & & \\
\hline \multirow[t]{4}{*}{2} & & 1 & & & & & 17 & 1 & & & & \\
\hline & & 2 & & & & & & 2 & 0.874 & & & 0.874 \\
\hline & & 3 & & & & & & 3 & 1.928 & & & 1.928 \\
\hline & & 4 & & & & & & 4 & & & & \\
\hline \multirow[t]{4}{*}{3} & & 1 & & & & & 18 & 1 & 8.361 & & & 8.361 \\
\hline & & 2 & & & & & & 2 & 3.301 & & & 3.301 \\
\hline & & 3 & & & & & & 3 & 0.701 & & & 0.701 \\
\hline & & 4 & & & & & & 4 & & & & \\
\hline \multirow[t]{4}{*}{4} & & 1 & & & & & 19 & 1 & & & & \\
\hline & & 2 & & & & & & 2 & & & & \\
\hline & & 3 & & & & & & 3 & & & & \\
\hline & & 4 & & & & & & 4 & & & & \\
\hline \multirow[t]{4}{*}{$\overline{5}$} & & 1 & & & & & 20 & 1 & & & & \\
\hline & & 2 & & & & & & 2 & & & & \\
\hline & & 3 & & & & & & 3 & & & & \\
\hline & & 4 & & & & & & 4 & & & & \\
\hline \multirow[t]{4}{*}{6} & & 1 & 0.898 & & & 0.898 & 21 & 1 & 4.967 & & & 4.967 \\
\hline & & 2 & 4.6367 & & & 4.6367 & & 2 & & & & \\
\hline & & 3 & 1.59235 & & & & & 3 & & & & \\
\hline & & 4 & & & & & & 4 & & & & \\
\hline \multirow[t]{4}{*}{7} & & 1 & 2.978 & & & 2.978 & 22 & 1 & 0.876 & & & 0.876 \\
\hline & & 2 & & & & & & 2 & & & & \\
\hline & & 3 & & & & & & 3 & 1.56 & & & 1.56 \\
\hline & & 4 & & & & & & 4 & & & & \\
\hline \multirow[t]{4}{*}{8} & & 1 & 2.277 & & & 2.277 & 23 & 1 & & & & \\
\hline & & 2 & & & & & & 2 & & & & \\
\hline & & 3 & & & & & & 3 & & & & \\
\hline & & 4 & & & & & & 4 & & & & \\
\hline \multirow[t]{4}{*}{$\overline{9}$} & & 1 & 2.155 & 2.32 & & 2.2375 & 24 & 1 & & & & \\
\hline & & 2 & & & & & & 2 & 9.084 & & & 9.084 \\
\hline & & 3 & & & & & & 3 & 1.715 & & & 1.715 \\
\hline & & 4 & & & & & & 4 & & & & \\
\hline 10 & & 1 & & & & & 25 & 1 & 1.08 & & & 1.08 \\
\hline & & 2 & & & & & & 2 & 1.641 & & & 1.641 \\
\hline & & 3 & & & & & & 3 & 1.035 & & & 1.035 \\
\hline & & 4 & & & & & & 4 & & & & \\
\hline 11 & & 1 & & 4.531 & & 4.531 & 26 & 1 & 4.169 & & & 4.169 \\
\hline & & 2 & & 5.423 & & 5.423 & & 2 & 1.965 & & & 1.965 \\
\hline & & 3 & & & & & & 3 & 10.226 & & & 10.226 \\
\hline & & 4 & & 2.972 & & 2.972 & & 4 & 0.298 & & & 0.298 \\
\hline 12 & & 1 & 1.432 & & & 1.432 & 27 & 1 & 4.035 & & & 4.035 \\
\hline & & 2 & & & & & & 2 & & & & \\
\hline & & 3 & & & & & & 3 & 1.678 & & & 1.678 \\
\hline & & 4 & & & & & & 4 & & & & \\
\hline 13 & & 1 & 0.908 & & & 0.908 & 28 & 1 & 1.381 & & & 1.381 \\
\hline & & 2 & & & & & & 2 & 1.065 & & & \\
\hline & & 3 & 0.7 & & & 0.7 & & 3 & 3.473 & & & 3.473 \\
\hline & & 4 & & & & & & 4 & & & & \\
\hline 14 & & 1 & & & & & 29 & 1 & & & & \\
\hline & & 2 & & & & & & 2 & & 7.951 & & 7.951 \\
\hline & & 3 & & & & & & 3 & 2.3027 & 2.666 & & 2.48435 \\
\hline & & 4 & & & & & & 4 & & & & \\
\hline 15 & & 1 & 0.983 & & & 0.983 & 30 & 1 & & & & \\
\hline & & 2 & & & & & & 2 & & & & \\
\hline & & 3 & & & & & & 3 & & & & \\
\hline & & 4 & & & & & & 4 & & & & \\
\hline
\end{tabular}




\begin{tabular}{|c|c|c|c|c|c|c|c|c|c|c|c|c|}
\hline \multicolumn{13}{|c|}{ Flight } \\
\hline \multicolumn{13}{|c|}{ Box 4} \\
\hline Parabola & Joint & & Colorization 1 & Colorization 2 & Colorization 3 & Average & Parabola & Joint & Colorization 1 & Colorization 2 & Colorization 3 & Average \\
\hline \multirow[t]{4}{*}{1} & 1 & 1 & & & & & 16 & 1 & & & & \\
\hline & & 2 & & & & & & 2 & 1.112351944 & & & 1.112352 \\
\hline & & 3 & & & & & & 3 & 6.003372191 & & & 6.003372 \\
\hline & & 4 & & & & & & 4 & 3.753877612 & & & 3.753878 \\
\hline \multirow[t]{4}{*}{2} & & 1 & & & & & 17 & 1 & & & & \\
\hline & & 2 & & & & & & 2 & & & & \\
\hline & & 3 & & & & & & 3 & 14.53177 & & & 14.53177 \\
\hline & & 4 & & & & & & 4 & 3.084408 & & & 3.084408 \\
\hline \multirow[t]{4}{*}{3} & & 1 & & & & & 18 & 1 & 4.338304 & & & 4.338304 \\
\hline & & 2 & & & & & & 2 & 1.95636 & & & 1.95636 \\
\hline & & 3 & & & & & & 3 & 32.92668 & & & 32.92668 \\
\hline & & 4 & & & & & & 4 & 6.70052 & 15.65752 & & 11.17902 \\
\hline \multirow[t]{4}{*}{4} & & 1 & & & & & 19 & 1 & 1.919401 & & & \\
\hline & & 2 & & & & & & 2 & 9.736357 & & & 9.736357 \\
\hline & & 3 & & & & & & 3 & & & & \\
\hline & & 4 & & & & & & 4 & & & & \\
\hline \multirow[t]{4}{*}{5} & & 1 & & & & & 20 & 1 & & & & \\
\hline & & 2 & & & & & & 2 & & & & \\
\hline & & 3 & & & & & & 3 & & & & \\
\hline & & 4 & & & & & & 4 & & & & \\
\hline \multirow[t]{4}{*}{6} & & 1 & & & & & 21 & 1 & & & & \\
\hline & & 2 & & & & & & 2 & & & & \\
\hline & & 3 & & & & & & 3 & & & & \\
\hline & & 4 & & & & & & 4 & & & & \\
\hline \multirow[t]{4}{*}{7} & & 1 & & & & & 22 & 1 & & & & \\
\hline & & 2 & & & & & & 2 & & & & \\
\hline & & 3 & & & & & & 3 & & & & \\
\hline & & 4 & & & & & & 4 & & & & \\
\hline \multirow[t]{4}{*}{8} & & 1 & 2.406 & & & 2.406 & 23 & 1 & 3.403561 & & & 3.403561 \\
\hline & & 2 & 1.737 & & & 1.737 & & 2 & 8.842582 & & & 8.842582 \\
\hline & & 3 & 1.358 & & & 1.358 & & 3 & 2.274266 & & & 2.274266 \\
\hline & & 4 & 1.334 & & & 1.334 & & 4 & 3.369182 & & & 3.369182 \\
\hline \multirow[t]{4}{*}{9} & & 1 & 1.763 & & & 1.763 & 24 & 1 & & & & \\
\hline & & 2 & 1.369 & 3.438 & & 2.4035 & & 2 & & & & \\
\hline & & 3 & 0.94 & 3.133 & & 2.0365 & & 3 & & & & \\
\hline & & 4 & 11.844 & 9.166 & & 10.505 & & 4 & & & & \\
\hline 10 & & 1 & 1.595 & 5.169 & & 3.382 & 25 & 1 & 3.528942 & & & 3.528942 \\
\hline & & 2 & 2.029 & 9.51 & & 5.7695 & & 2 & 2.978903 & & & 2.978903 \\
\hline & & 3 & & 12.287 & & 12.287 & & 3 & 15.29944 & & & 15.29944 \\
\hline & & 4 & & & & & & 4 & 15.66097 & & & 15.66097 \\
\hline 11 & & 1 & 11.379 & 3.68 & & 7.5295 & 26 & 1 & & & & \\
\hline & & 2 & 0.938 & 3.841 & & 2.3895 & & 2 & 10.68791 & & & 10.68791 \\
\hline & & 3 & 1.223 & 4.705 & & 2.964 & & 3 & 1.825198 & & & 1.825198 \\
\hline & & 4 & 1.008 & 7.359 & & 4.1835 & & 4 & 5.160568 & & & 5.160568 \\
\hline 12 & & 1 & 1.958 & & & 1.958 & 27 & 1 & 18.2097 & & & 18.2097 \\
\hline & & 2 & 1.398 & & & 1.398 & & 2 & 3.234621 & & & 3.234621 \\
\hline & & 3 & 1.617 & & & & & 3 & 1.584644 & & & 1.584644 \\
\hline & & 4 & & & & & & 4 & & & & \\
\hline 13 & & 1 & & & & & 28 & 1 & 18.2097 & & & 18.2097 \\
\hline & & 2 & & & & & & 2 & 3.234621 & & & 3.234621 \\
\hline & & 3 & & & & & & 3 & & & & \\
\hline & & 4 & & & & & & 4 & 14.46775 & & & 14.46775 \\
\hline 14 & & 1 & 1.774 & & & 1.774 & 29 & 1 & & 3.674 & & 3.674 \\
\hline & & 2 & 1.734 & & & 1.734 & & 2 & & & & \\
\hline & & 3 & 1.338 & & & 1.338 & & 3 & & 3.774 & & 3.774 \\
\hline & & 4 & 2.101 & & & 2.101 & & 4 & & 12.228 & & 12.228 \\
\hline 15 & & 1 & 0.951 & & & 0.951 & 30 & 1 & & & & \\
\hline & & 2 & 1.164010545 & & & 1.164011 & & 2 & & & & \\
\hline & & 3 & 1.087996039 & & & 1.087996 & & 3 & & & & \\
\hline & & 4 & 1.152402147 & & & 1.152402 & & 4 & & & & \\
\hline
\end{tabular}




\section{Ground sample porosity data}

\begin{tabular}{|c|c|c|c|c|c|c|c|c|c|c|c|c|}
\hline \multicolumn{13}{|c|}{ Ground } \\
\hline \multicolumn{13}{|c|}{ Box 1} \\
\hline Parabola & Joint & & Colorization 1 & Colorization 2 & Colorization 3 & Average & Parabola & Joint & Colorization 1 & Colorization 2 & Colorization 3 & Average \\
\hline \multirow{4}{*}{\multicolumn{2}{|c|}{1}} & 1 & & & & \multirow{3}{*}{0.602944} & 16 & 1 & & & & \\
\hline & & 2 & 0.602944 & & & & & 2 & 0.948784 & & & 0.948784 \\
\hline & & 3 & & & & & & 3 & & & & \\
\hline & & 4 & 0.782945 & & & 0.782945 & & 4 & 2.377487 & & & 2.377487 \\
\hline \multirow[t]{4}{*}{2} & & 1 & 1.217721 & & & 1.217721 & 17 & 1 & & & & \\
\hline & & 2 & & & & & & 2 & 1.430875 & & & 1.430875 \\
\hline & & 3 & 0.854789 & & & 0.854789 & & 3 & 4.27064 & & & 4.27064 \\
\hline & & 4 & & & & & & 4 & & & & \\
\hline \multirow{4}{*}{\multicolumn{2}{|c|}{3}} & 1 & 0.968708 & & & 0.968708 & 18 & 1 & & & & \\
\hline & & 2 & 1.354235 & & & 1.354235 & & 2 & & & & \\
\hline & & 3 & 1.366349 & & & 1.366349 & & 3 & & & & \\
\hline & & 4 & & & & & & 4 & & & & \\
\hline \multirow[t]{4}{*}{4} & & 1 & & & & & 19 & 1 & & & & \\
\hline & & 2 & & & & & & 2 & 1.31777 & & & 1.31777 \\
\hline & & 3 & & & & & & 3 & & & & \\
\hline & & 4 & & & & & & 4 & & & & \\
\hline \multirow[t]{4}{*}{5} & & 1 & & & & & 20 & 1 & & & & \\
\hline & & 2 & & & & & & 2 & 8.870811 & & & 8.870811 \\
\hline & & 3 & & & & & & 3 & & & & \\
\hline & & 4 & & & & & & 4 & & & & \\
\hline \multirow[t]{4}{*}{6} & & 1 & & & & & 21 & 1 & & & & \\
\hline & & 2 & & & & & & 2 & & & & \\
\hline & & 3 & & & & & & 3 & & & & \\
\hline & & 4 & & & & & & 4 & 0.827364 & & & 0.827364 \\
\hline \multirow[t]{4}{*}{7} & & 1 & & & & & 22 & 1 & 1.295624 & & & 1.295624 \\
\hline & & 2 & & & & & & 2 & 5.783986 & & & 5.783986 \\
\hline & & 3 & & & & & & 3 & & & & \\
\hline & & 4 & & & & & & 4 & 19.9299 & & & 19.9299 \\
\hline \multirow[t]{4}{*}{8} & & 1 & & & & & 23 & 1 & & & & \\
\hline & & 2 & 1.040277 & & & 1.040277 & & 2 & 1.95599 & & & 1.95599 \\
\hline & & 3 & 1.117631 & & & 1.117631 & & 3 & & & & \\
\hline & & 4 & & & & & & 4 & 1.657469 & & & 1.657469 \\
\hline 9 & & 1 & & & & & 24 & 1 & & & & \\
\hline & & 2 & 0.609908 & & & 0.609908 & & 2 & 9.678224 & & & 9.678224 \\
\hline & & 3 & 0.655646 & & & 0.655646 & & 3 & & & & \\
\hline & & 4 & 1.62733 & & & 1.62733 & & 4 & & & & \\
\hline 10 & & 1 & 0.858855 & & & 0.858855 & 25 & 1 & & & & \\
\hline & & 2 & & & & & & 2 & & & & \\
\hline & & 3 & 0.7466 & & & 0.7466 & & 3 & & & & \\
\hline & & 4 & & & & & & 4 & & & & \\
\hline 11 & & 1 & & & & & 26 & 1 & & & & \\
\hline & & 2 & & & & & & 2 & & & & \\
\hline & & 3 & & & & & & 3 & 6.866098 & & & 6.866098 \\
\hline & & 4 & & & & & & 4 & 2.946452 & & & 2.946452 \\
\hline 12 & & 1 & & & & & 27 & 1 & & & & \\
\hline & & 2 & & & & & & 2 & & & & \\
\hline & & 3 & & & & & & 3 & & & & \\
\hline & & 4 & & & & & & 4 & & & & \\
\hline 13 & & 1 & 1.064222 & & & 1.064222 & 28 & 1 & & & & \\
\hline & & 2 & & & & & & 2 & 1.005142 & & & 1.005142 \\
\hline & & 3 & & & & & & 3 & & & & \\
\hline & & 4 & & & & & & 4 & 2.642414 & & & 2.642414 \\
\hline 14 & & 1 & & & & & 29 & 1 & & & & \\
\hline & & 2 & & & & & & 2 & 15.35625 & & & 15.35625 \\
\hline & & 3 & & & & & & 3 & 14.72851 & & & 14.72851 \\
\hline & & 4 & & & & & & 4 & 4.866372 & & & 4.866372 \\
\hline 15 & & 1 & & & & & 30 & 1 & & & & \\
\hline & & 2 & & & & & & 2 & & & & \\
\hline & & 3 & 0.9248784 & & & 0.924878 & & 3 & & & & \\
\hline & & 4 & 0.73321 & & & 0.73321 & & 4 & & & & \\
\hline
\end{tabular}




\begin{tabular}{|c|c|c|c|c|c|c|c|c|c|c|c|c|}
\hline \multicolumn{13}{|c|}{ Ground } \\
\hline \multicolumn{13}{|c|}{ Box 2} \\
\hline Parabola & Joint & & Colorization 1 & Colorization 2 & Colorization 3 & Average & Parabola & Joint & Colorization 1 & Colorization 2 & Colorization 3 & Average \\
\hline \multirow[t]{4}{*}{1} & 1 & 1 & 2.06981 & & & 2.06981 & 16 & 1 & 10.04864 & & & 10.04864 \\
\hline & & 2 & & & & & & 2 & 2.22487 & & & 2.22487 \\
\hline & & 3 & 1.234968 & & & 1.234968 & & 3 & & & & \\
\hline & & 4 & & & & & & 4 & & & & \\
\hline \multirow{4}{*}{\multicolumn{2}{|c|}{2}} & 1 & & & & & 17 & 1 & & & & \\
\hline & & 2 & & & & & & 2 & & & & \\
\hline & & 3 & 1.531662 & & & 1.531662 & & 3 & & & & \\
\hline & & 4 & 1.671349 & & & 1.671349 & & 4 & & & & \\
\hline \multirow[t]{4}{*}{3} & & 1 & & & & & 18 & 1 & & & & \\
\hline & & 2 & & & & & & 2 & & & & \\
\hline & & 3 & 1.113897 & & & 1.113897 & & 3 & & & & \\
\hline & & 4 & & & & & & 4 & & & & \\
\hline \multirow[t]{4}{*}{4} & & 1 & & & & & 19 & 1 & 2.3541 & & & 2.3541 \\
\hline & & 2 & & & & & & 2 & 2.7228 & & & 2.7228 \\
\hline & & 3 & & & & & & 3 & & & & \\
\hline & & 4 & & & & & & 4 & & & & \\
\hline \multirow[t]{4}{*}{5} & & 1 & & & & & 20 & 1 & 18.59845 & & & 18.59845 \\
\hline & & 2 & 0.941724 & & & 0.941724 & & 2 & 1.943692 & & & 1.943692 \\
\hline & & 3 & 2.753008 & & & 2.753008 & & 3 & 0.117949 & & & 0.117949 \\
\hline & & 4 & 1.171217 & & & 1.171217 & & 4 & & & & \\
\hline \multirow[t]{4}{*}{6} & & 1 & & & & & 21 & 1 & 1.80859 & & & 1.80859 \\
\hline & & 2 & 1.83669508 & & & 1.836695 & & 2 & 1.12437 & & & 1.12437 \\
\hline & & 3 & 0.950950951 & & & 0.950951 & & 3 & & & & \\
\hline & & 4 & & & & & & 4 & & & & \\
\hline \multirow[t]{4}{*}{7} & & 1 & 2.049172 & & & 2.049172 & 22 & 1 & 2.282117 & & & 2.282117 \\
\hline & & 2 & 1.929539 & & & 1.929539 & & 2 & & & & \\
\hline & & 3 & & & & & & 3 & & & & \\
\hline & & 4 & & & & & & 4 & & & & \\
\hline \multirow[t]{4}{*}{8} & & 1 & 2.021938 & & & 2.021938 & 23 & 1 & 21.2695 & & & 21.2695 \\
\hline & & 2 & 2.981079 & & & 2.981079 & & 2 & 13.4537 & & & 13.4537 \\
\hline & & 3 & & & & & & 3 & & & & \\
\hline & & 4 & & & & & & 4 & & & & \\
\hline \multirow[t]{4}{*}{9} & & 1 & & & & & 24 & 1 & & & & \\
\hline & & 2 & 11.02722 & & & 11.02722 & & 2 & 1.155074 & & & 1.155074 \\
\hline & & 3 & 0.577157 & & & 0.577157 & & 3 & 1.228622 & & & 1.228622 \\
\hline & & 4 & & & & & & 4 & & & & \\
\hline 10 & & 1 & & & & & 25 & 1 & & & & \\
\hline & & 2 & & & & & & 2 & & & & \\
\hline & & 3 & & & & & & 3 & & & & \\
\hline & & 4 & & & & & & 4 & & & & \\
\hline 11 & & 1 & & & & & 26 & 1 & 1.119403 & & & 1.119403 \\
\hline & & 2 & & & & & & 2 & 1.282312 & & & 1.282312 \\
\hline & & 3 & & & & & & 3 & 12.95981 & & & 12.95981 \\
\hline & & 4 & & & & & & 4 & & & & \\
\hline 12 & & 1 & 0.627803 & & & 0.627803 & 27 & 1 & & & & \\
\hline & & 2 & 0.935297 & & & 0.935297 & & 2 & & & & \\
\hline & & 3 & 1.183679 & & & 1.183679 & & 3 & & & & \\
\hline & & 4 & 1.502708 & & & 1.502708 & & 4 & & & & \\
\hline 13 & & 1 & & & & & 28 & 1 & & & & \\
\hline & & 2 & & & & & & 2 & & & & \\
\hline & & 3 & & & & & & 3 & & & & \\
\hline & & 4 & & & & & & 4 & & & & \\
\hline 14 & & 1 & 3.486776 & & & 3.486776 & 29 & 1 & & & & \\
\hline & & 2 & 1.022587 & & & 1.022587 & & 2 & & & & \\
\hline & & 3 & & & & & & 3 & & & & \\
\hline & & 4 & & & & & & 4 & & & & \\
\hline 15 & & 1 & & & & & 30 & 1 & & & & \\
\hline & & 2 & & & & & & 2 & & & & \\
\hline & & 3 & 10.14131 & & & 10.14131 & & 3 & & & & \\
\hline & & 4 & & & & & & 4 & & & & \\
\hline
\end{tabular}




\begin{tabular}{|c|c|c|c|c|c|c|c|c|c|c|c|c|c|}
\hline \multicolumn{14}{|c|}{ Ground } \\
\hline \multicolumn{14}{|c|}{ Box 3} \\
\hline Parabola & Joint & & Colorization 1 & Colorization 2 & Colorization 3 & Average & Parabola & Joint & & Colorization 1 & Colorization 2 & Colorization 3 & Average \\
\hline \multirow{4}{*}{\multicolumn{2}{|c|}{1}} & 1 & & & & & 16 & & 1 & & & & \\
\hline & & 2 & & & & & & & 2 & & & & \\
\hline & & 3 & & & & & & & 3 & & & & \\
\hline & & 4 & & & & & & & 4 & & & & \\
\hline \multirow[t]{4}{*}{2} & & 1 & & & & & 17 & & 1 & & & & \\
\hline & & 2 & & & & & & & 2 & 0.788183379 & & & 0.788183 \\
\hline & & 3 & & & & & & & 3 & & & & \\
\hline & & 4 & & & & & & & 4 & & & & \\
\hline \multirow[t]{4}{*}{3} & & 1 & & & & & 18 & & 1 & & & & \\
\hline & & 2 & & & & & & & 2 & & & & \\
\hline & & 3 & 2.184164671 & & & 2.184165 & & & 3 & & & & \\
\hline & & 4 & & & & & & & 4 & & & & \\
\hline \multirow[t]{4}{*}{4} & & 1 & & & & & 19 & & 1 & & & & \\
\hline & & 2 & & & & & & & 2 & & & & \\
\hline & & 3 & & & & & & & 3 & & & & \\
\hline & & 4 & & & & & & & 4 & & & & \\
\hline \multirow[t]{4}{*}{5} & & 1 & & & & & 20 & & 1 & & & & \\
\hline & & 2 & & & & & & & 2 & & & & \\
\hline & & 3 & & & & & & & 3 & & & & \\
\hline & & 4 & & & & & & & 4 & & & & \\
\hline \multirow[t]{4}{*}{6} & & 1 & & & & & 21 & & 1 & & & & \\
\hline & & 2 & & & & & & & 2 & & & & \\
\hline & & 3 & & & & & & & 3 & & & & \\
\hline & & 4 & & & & & & & 4 & & & & \\
\hline \multirow[t]{4}{*}{7} & & 1 & & & & & 22 & & 1 & & & & \\
\hline & & 2 & & & & & & & 2 & & & & \\
\hline & & 3 & & & & & & & 3 & & & & \\
\hline & & 4 & & & & & & & 4 & & & & \\
\hline \multirow[t]{4}{*}{8} & & 1 & & & & & 23 & & 1 & & & & \\
\hline & & 2 & & & & & & & 2 & & & & \\
\hline & & 3 & & & & & & & 3 & & & & \\
\hline & & 4 & & & & & & & 4 & & & & \\
\hline \multirow[t]{4}{*}{9} & & 1 & & & & & 24 & & 1 & & & & \\
\hline & & 2 & & & & & & & 2 & & & & \\
\hline & & 3 & & & & & & & 3 & & & & \\
\hline & & 4 & & & & & & & 4 & & & & \\
\hline 10 & & 1 & & & & & 25 & & 1 & & & & \\
\hline & & 2 & & & & & & & 2 & & & & \\
\hline & & 3 & 1.993850386 & & & 1.99385 & & & 3 & & & & \\
\hline & & 4 & 1.579209271 & & & 1.579209 & & & 4 & & & & \\
\hline 11 & & 1 & & & & & 26 & & 1 & & & & \\
\hline & & 2 & & & & & & & 2 & & & & \\
\hline & & 3 & & & & & & & 3 & & & & \\
\hline & & 4 & & & & & & & 4 & & & & \\
\hline 12 & & 1 & & & & & 27 & & 1 & & & & \\
\hline & & 2 & & & & & & & 2 & & & & \\
\hline & & 3 & & & & & & & 3 & & & & \\
\hline & & 4 & & & & & & & 4 & & & & \\
\hline 13 & & 1 & & & & & 28 & & 1 & 0.724877267 & & & 0.724877 \\
\hline & & 2 & & & & & & & 2 & & & & \\
\hline & & 3 & & & & & & & 3 & & & & \\
\hline & & 4 & & & & & & & 4 & 1.557585552 & & & 1.557586 \\
\hline 14 & & 1 & & & & & 29 & & 1 & & & & \\
\hline & & 2 & & & & & & & 2 & 5.001494011 & & & 5.001494 \\
\hline & & 3 & & & & & & & 3 & & & & \\
\hline & & 4 & & & & & & & 4 & 0.903591389 & & & 0.903591 \\
\hline 15 & & 1 & & & & & 30 & & 1 & & & & \\
\hline & & 2 & & & & & & & 2 & & & & \\
\hline & & 3 & & & & & & & 3 & & & & \\
\hline & & 4 & & & & & & & 4 & & & & \\
\hline
\end{tabular}




\section{Appendix F: Magnetic Field Plots and Data}

The magnet field on the hand soldering boards was measured after the flight. To get a magnetic field profile the magnetic field was measured at five points on each board, the four corners and the center of the board. The results are presented below, beneath a diagram of the measurement locations. Ground box 2 was the same box as flight box 2 , but with the sample boards replaced, for this reason these two boxes have the same magnetic profile.

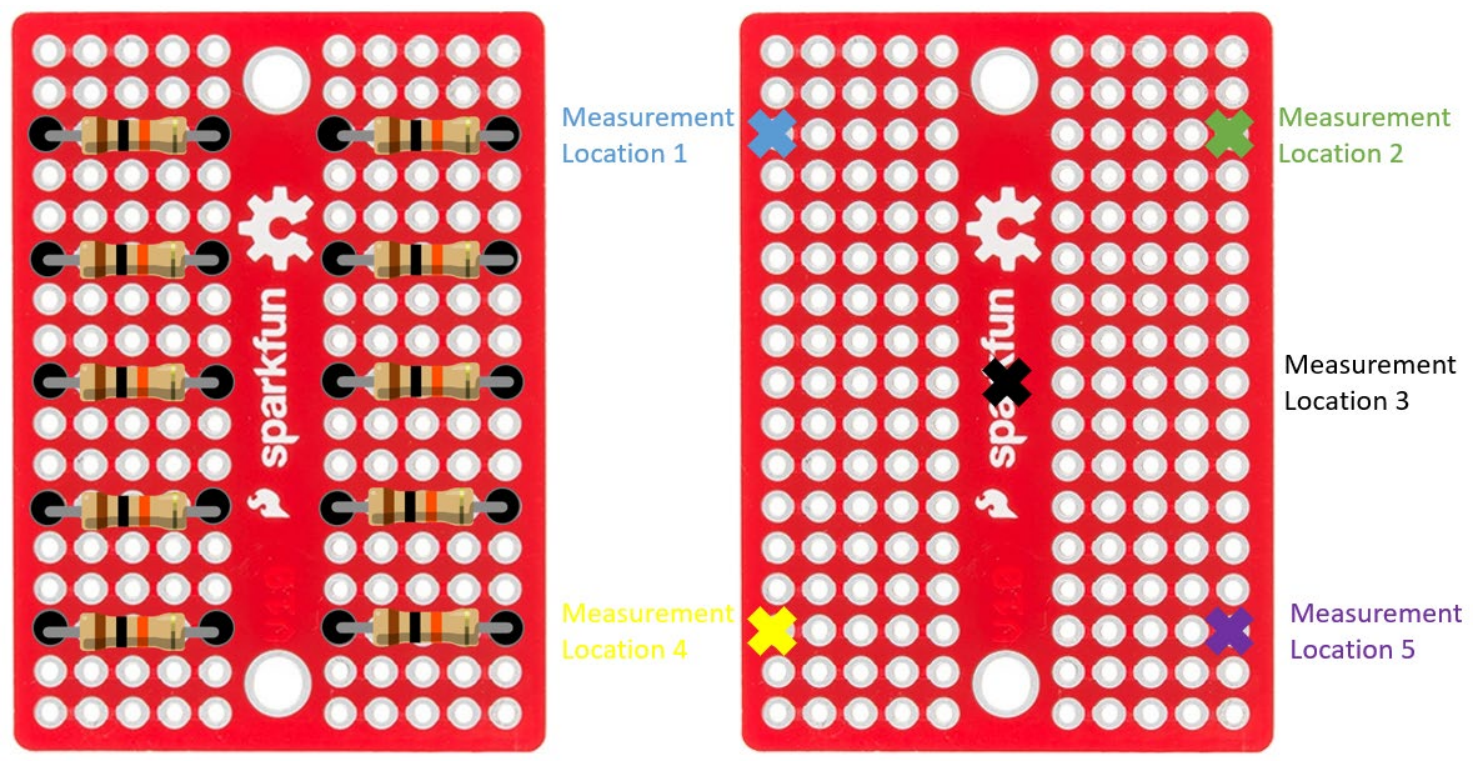




\begin{tabular}{|c|c|c|c|c|c|c|}
\hline & & Location & Measurement (mT) & & Location & Measurement (mT) \\
\hline \multirow{15}{*}{$\begin{array}{l}\text { Flight } \\
\text { box } 2 / \\
\text { Ground } \\
\text { box } 2\end{array}$} & \multirow{5}{*}{ Board 1} & 1 & 13.6 & \multirow{5}{*}{ Board 4} & 1 & 12.4 \\
\hline & & 2 & 16.8 & & 2 & 19.9 \\
\hline & & 3 & 50.7 & & 3 & 61.2 \\
\hline & & 4 & 17.5 & & 4 & 17 \\
\hline & & 5 & 18.5 & & 5 & 22.9 \\
\hline & \multirow{5}{*}{ Board 2} & 1 & 23 & \multirow{5}{*}{ Board 5} & 1 & 12.1 \\
\hline & & 2 & 24 & & 2 & 20.2 \\
\hline & & 3 & 52.2 & & 3 & 60.7 \\
\hline & & 4 & 21.4 & & 4 & 23 \\
\hline & & 5 & 12.9 & & 5 & 25.7 \\
\hline & \multirow{5}{*}{ Board 3} & 1 & 16.2 & \multirow{5}{*}{ Board 6} & 1 & 20.2 \\
\hline & & 2 & 15.4 & & 2 & 25.2 \\
\hline & & 3 & 49.2 & & 3 & 57.8 \\
\hline & & 4 & 26.4 & & 4 & 19.7 \\
\hline & & 5 & 29 & & 5 & 19.9 \\
\hline
\end{tabular}

\begin{tabular}{|c|c|c|c|c|c|c|}
\hline & & Location & Measurement (mT) & & Location & Measurement (mT) \\
\hline \multirow{15}{*}{$\begin{array}{l}\text { Flight } \\
\text { box } 3\end{array}$} & \multirow{5}{*}{ Board 1} & 1 & 18.2 & \multirow{5}{*}{ Board 4} & 1 & 20 \\
\hline & & 2 & 12.3 & & 2 & 27.7 \\
\hline & & 3 & 52.6 & & 3 & 65.7 \\
\hline & & 4 & 24.1 & & 4 & 14.5 \\
\hline & & 5 & 27.1 & & 5 & 19.1 \\
\hline & \multirow{5}{*}{ Board 2} & 1 & 14.4 & \multirow{5}{*}{ Board 5} & 1 & 21 \\
\hline & & 2 & 14.6 & & 2 & 26.4 \\
\hline & & 3 & 56.9 & & 3 & 64.1 \\
\hline & & 4 & 31.6 & & 4 & 13.1 \\
\hline & & 5 & 27 & & 5 & 20.7 \\
\hline & \multirow{5}{*}{ Board 3} & 1 & 20.4 & \multirow{5}{*}{ Board 6} & 1 & 16.7 \\
\hline & & 2 & 17.8 & & 2 & 24.8 \\
\hline & & 3 & 63.9 & & 3 & 64.2 \\
\hline & & 4 & 27.2 & & 4 & 18.1 \\
\hline & & 5 & 25.5 & & 5 & 24.4 \\
\hline
\end{tabular}




\begin{tabular}{|c|c|c|c|c|c|c|}
\hline & & Location & Measurement (mT) & & Location & Measurement (mT) \\
\hline \multirow{15}{*}{$\begin{array}{l}\text { Flight } \\
\text { box } 4\end{array}$} & \multirow{5}{*}{ Board 1} & 1 & 17.7 & \multirow{5}{*}{ Board 4} & 1 & 18.5 \\
\hline & & 2 & 16.6 & & 2 & 19.3 \\
\hline & & 3 & 57.8 & & 3 & 85 \\
\hline & & 4 & 27.9 & & 4 & 20.7 \\
\hline & & 5 & 21 & & 5 & 26.5 \\
\hline & \multirow{5}{*}{ Board 2} & 1 & 19.1 & \multirow{5}{*}{ Board 5} & 1 & 6.9 \\
\hline & & 2 & 14.8 & & 2 & 18.1 \\
\hline & & 3 & 64.4 & & 3 & 68.5 \\
\hline & & 4 & 23.5 & & 4 & 20.2 \\
\hline & & 5 & 22.9 & & 5 & 21.1 \\
\hline & \multirow{5}{*}{ Board 3} & 1 & 21.9 & \multirow{5}{*}{ Board 6} & 1 & 26.4 \\
\hline & & 2 & 13.9 & & 2 & 26.4 \\
\hline & & 3 & 72.2 & & 3 & 76.2 \\
\hline & & 4 & 26.1 & & 4 & 16.8 \\
\hline & & 5 & 26.6 & & 5 & 19.2 \\
\hline
\end{tabular}




\section{Appendix G: Matlab Image Processing Code}

There are two MATAB codes used to process the tricolor images, one that process the images to get the intended color of each pixel and make sure that it shows the max color values for that color (code 2) and another (code 1) that pulls the images to feed into that function and calculate the voidage based on the ratio on red (voids) space to white (solder) in the resultant image.

\section{Code 1: Processing Script}

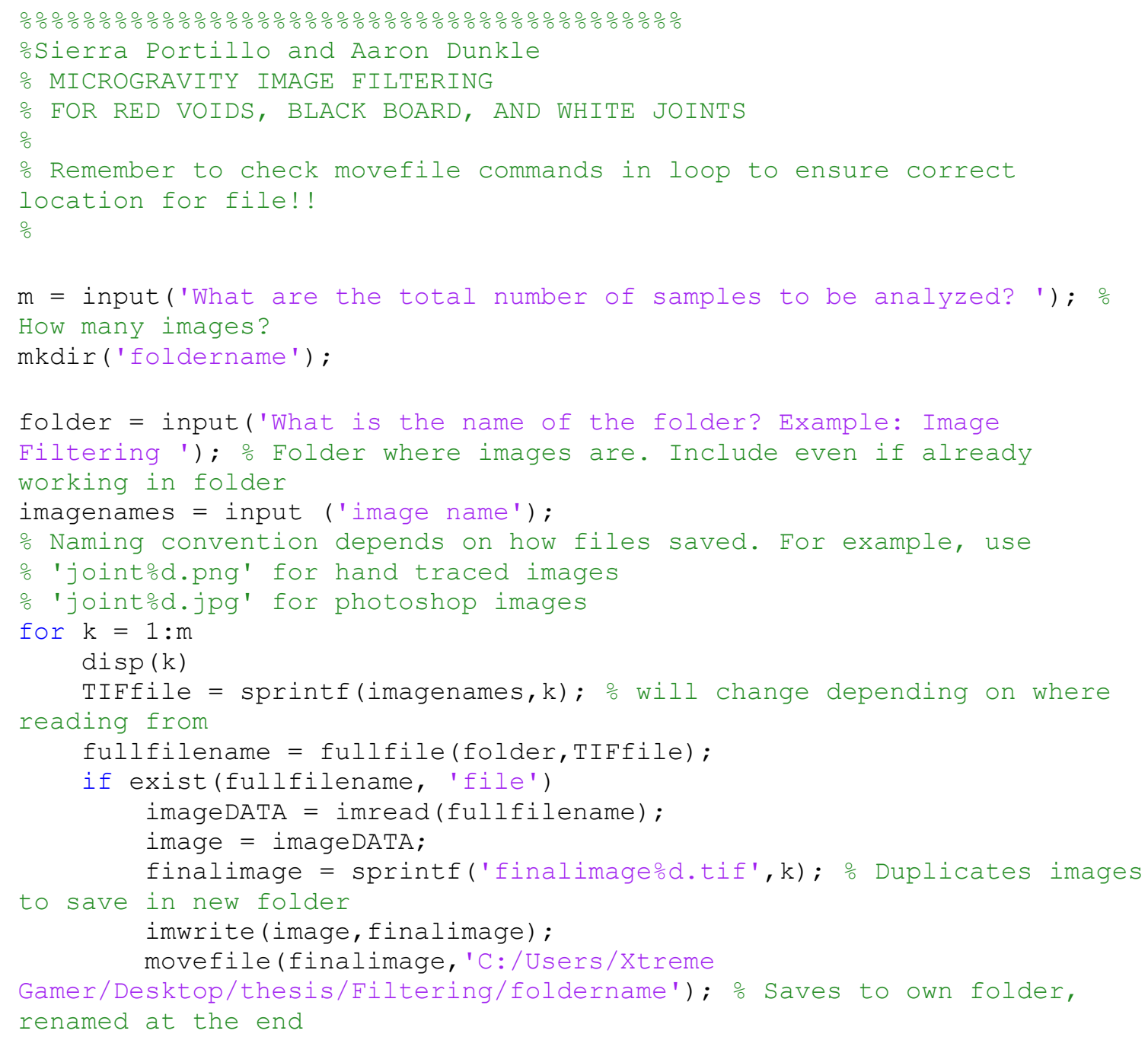




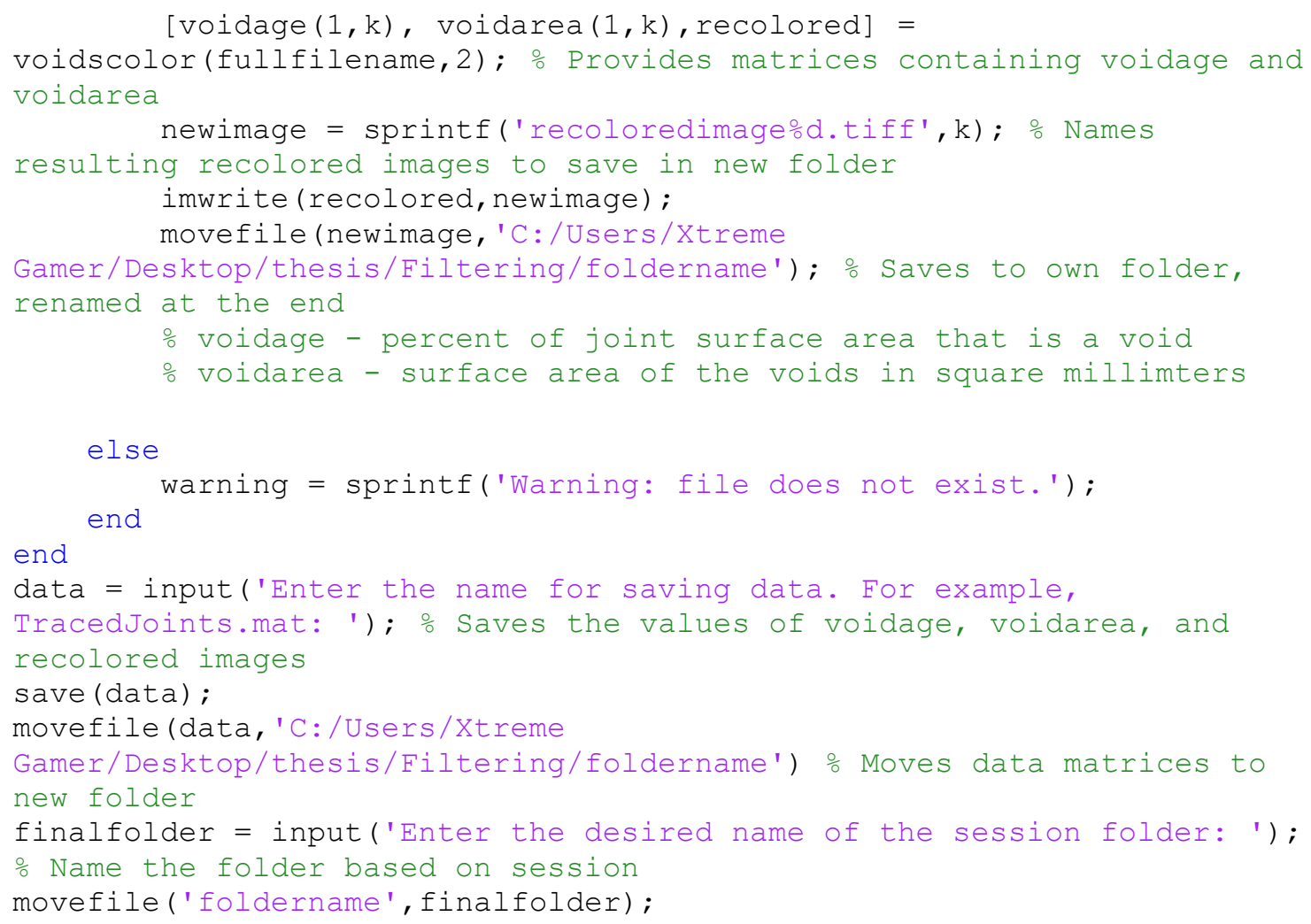

\section{Code 2: Image Preprocessing Function}

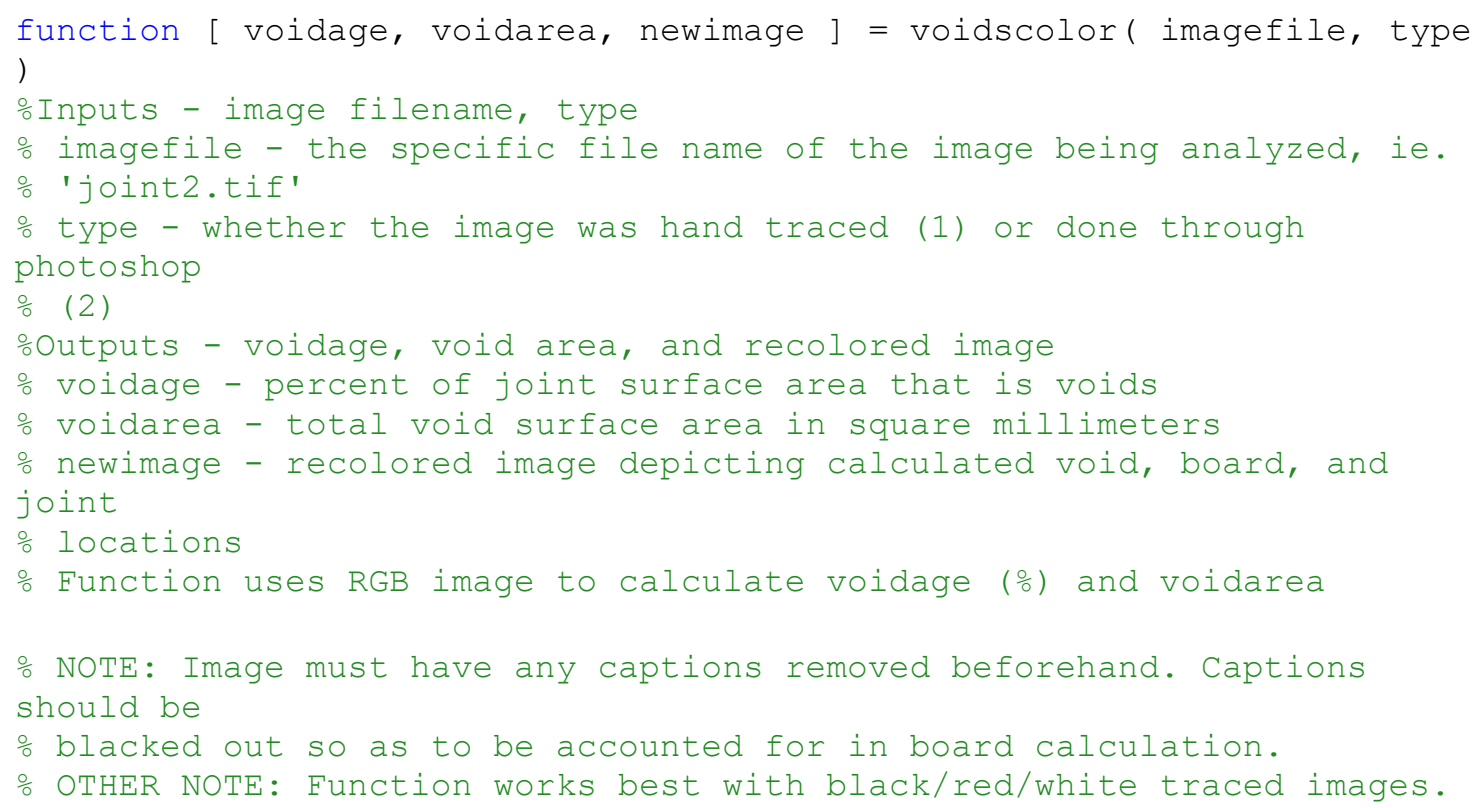




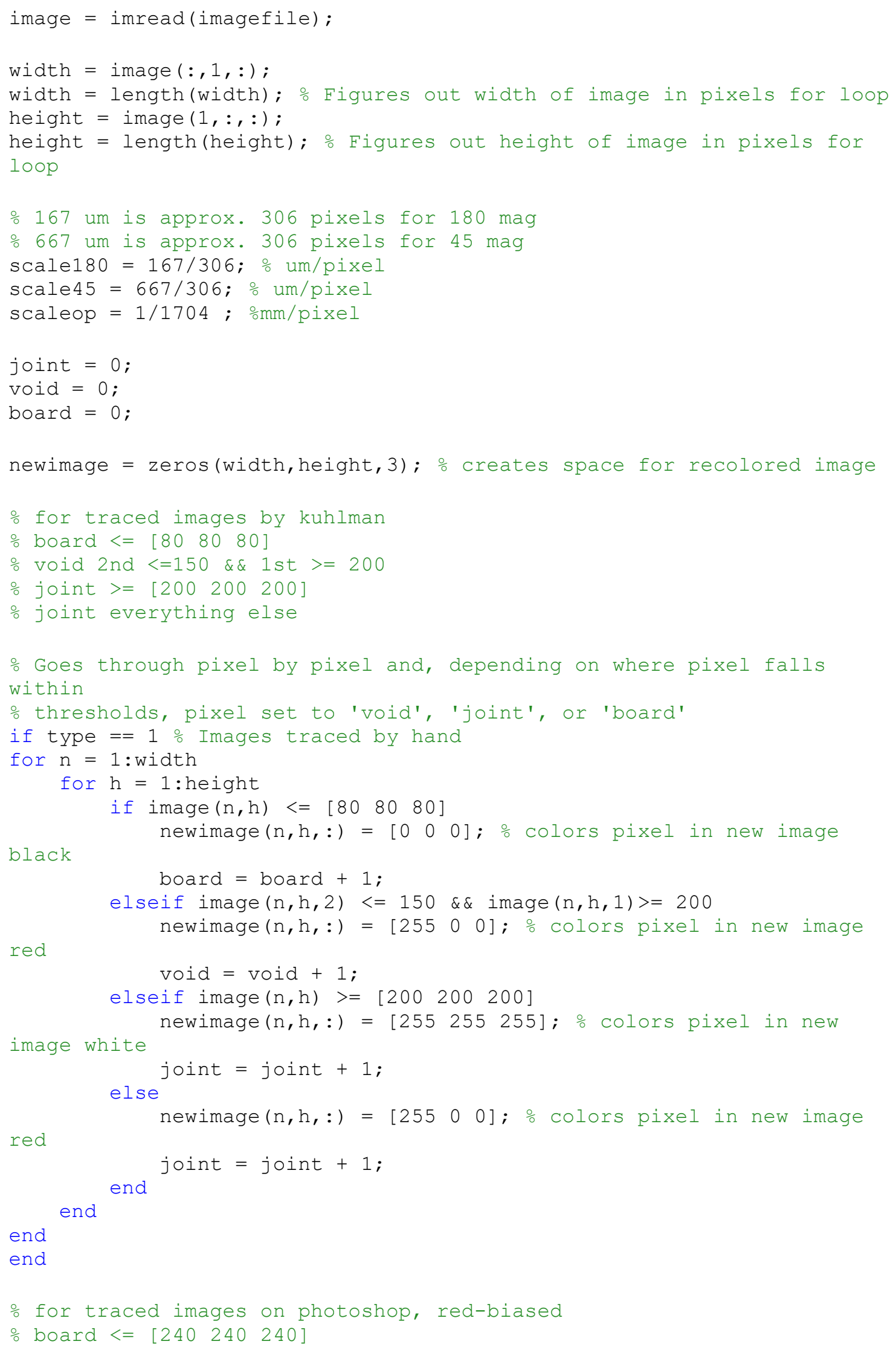




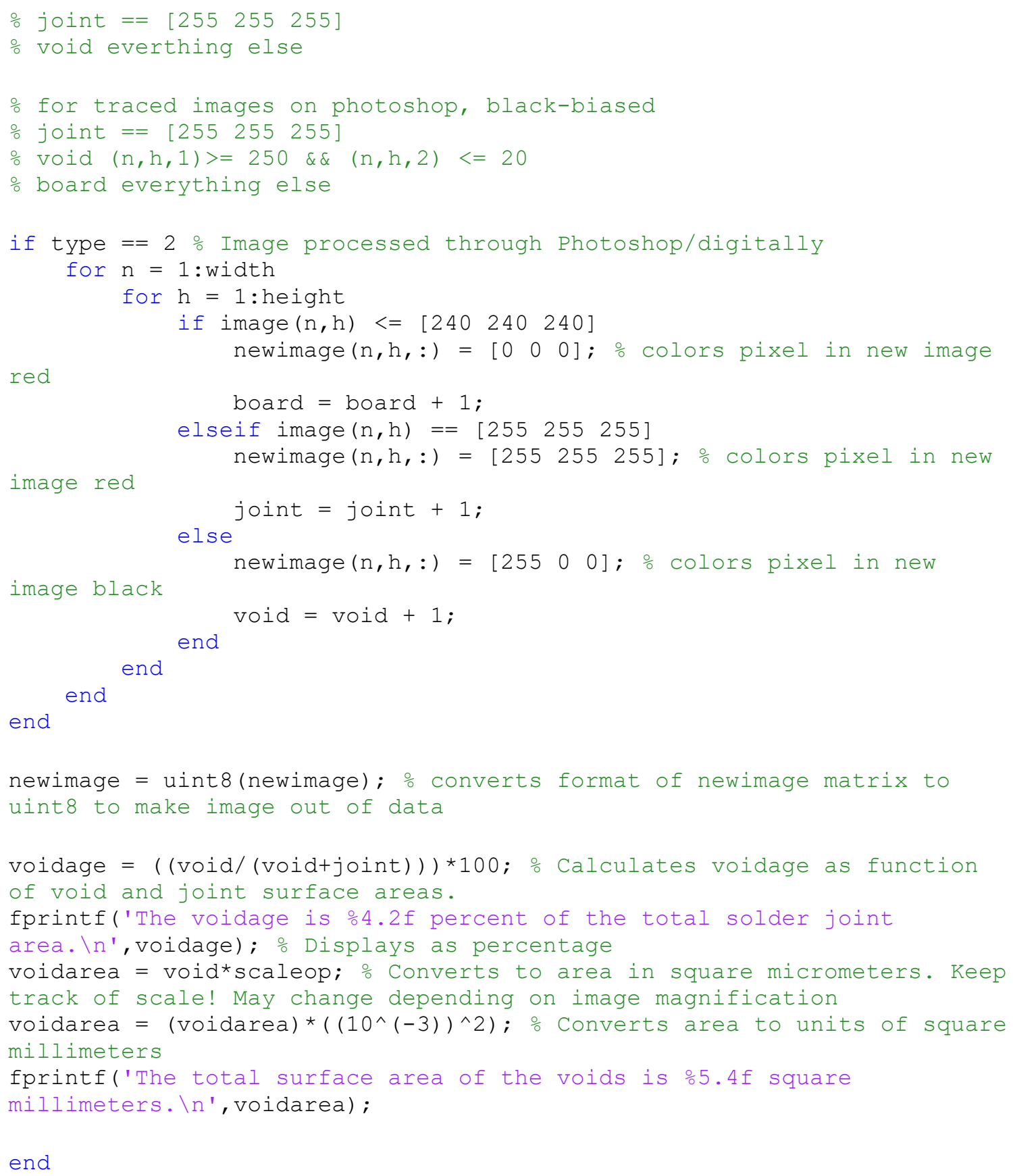

